\title{
Bifurcação de pontos de equilíbrio em sistemas acoplados com simetria do tipo produto coroa
}

\author{
Luci Any Francisco Roberto ${ }^{1}$
}

Orientador: Profa. Dra. Miriam Garcia Manoel

Dissertação apresentada ao Instituto de Ciências Matemáticas e de Computação da Universidade de São Paulo, como parte dos requisitos para obtenção do título de Mestre em ciências - Área: Matemática.

\author{
USP - São Carlos \\ Fevereiro/2004
}

\footnotetext{
${ }^{1}$ Este trabalho teve suporte financeiro da Fapesp proc: 01/12533-6
} 
A Comissão Julgadora:

Profa. Dra. Miriam Garcia Manoel

Profa. Dra. Isabel Salgado Labouriau thahousides 
Ao meu Pai Diorando, por ensinar-me $a$ aprender sempre, ̀̀ minha mãe Helena, pelo exemplo de dedicação, e à minha irmã Ligia pelo amor e carinho. 


\section{Agradecimentos}

Agradeço a Deus por minha existência pois, através dela, pude rcalizar este trabalho.

À minha orientadora Prof. Dra. Miriam Garcia Manoel pelos ensinamentos, confiança c fundamental orientação no desenvolvimento deste trabalho. Obrigada pela confiança transmitida e principalmente, por acreditar em mim!

Em especial ao meu namorado Marcelo pelo amor, carinho, compreensão, apoio e por suportar o meu mau hurnor nas horas dificeis.

Aos meus Pais pclo grande apoio, compreensão, por sofrerem comigo nas minhas dificuldades, por torcerem por mim e mais do que tudo, pelo infinito amor, quero compartilhar com vocês minha extrema alegria.

A minha querida irmã, Ligia por estar sempre me apoiando, torcendo por mim e cujo carinho e amor me reergucu o me deu forças.

Aos meus amigos Flávia, Mariana, Graziclle, Mario Ronaldinho, Aline, Fábio e Alex por tudo que eles me proporcionaram com suas atenções, amizades e carinhos. Valeu pessoal!!!

À minha amiga Patrícia pelo companherismo e apoio que inúmeras vezes precisei.

A todos os professores e funcionários do Instituto de Ciências Matemáticas e de Computação peła gentileza e atenção diariamente prestados.

A todos das escolas E.E.P.G. Profa. Dirce de Almeida Braga Wrasek e E.E.P.S.G. Coripheu de Azcvedo Marques que sempre me incentivaram para que eu concluísse esta etapa da minha vida.

A Fapesp pela bolsa de estudo concedida.

Enfirn, a todos aqueles que colaboraram de alguma forma para a realização deste trabalho. 


\section{Resumo}

Neste projeto estudamos bifurcações de pontos de equilíbrio em sistemas de $N$ células acopladas que possuen un grupo de simetria "global" $\mathcal{G}$ c cada célula possui sua simetria "interna" $\mathcal{L}$, onde $\mathcal{G}$ é um subgrupo do grupo $\mathrm{S}_{N}$ clas permulações de $N$ elementos e $\mathcal{L}$ é um grupo de Lie compacto. O acoplamento que consideramos é invariante segundo as simetrias internas de cada cólula; neste caso, a combinação dos grupos $\mathcal{L}$ e $\mathcal{G}$ que leva à simetria total do sistenla é a do grupo $\mathcal{L}$ produto coroa $\mathcal{G}, \mathcal{L}$ l $\mathcal{G}$, ou seja, $\mathcal{L}^{N}+\mathcal{G}$. Relacionamos as bifureagoes de pontos de equilín)ro que ocorrem en sistemas acoplados com grupo de simetria $\mathcal{L}<\mathcal{G}$ às bifurcações com simetria $\mathcal{L}$ ou $\mathcal{G}$. Fazemos um aplicação dos resultados obtidos para un caso não degenerado de $N$ células acoplaclas com sinetria $\mathrm{O}(2)$ \ $\mathbf{S}_{N}$. Vemos como a teoria invariante para $\mathrm{O}(2)$ < $\mathbf{S}_{N}$ está relacionada às teorias invariantes para os grupos $\mathrm{O}(2)$ e $\mathrm{S}_{N}$. Verificamos que, a menos de conjugação, existem exatamente $N$ ramos de soluções, a sabcr, as com subgrupos de isotropias axiais. Além disso, discutimos a estabilidade das soluções e dircção dos ramos. 


\begin{abstract}
In this project we study steady-state bifurcation in system of $N$ coupled cells that possess a "global" symmetry group $\mathcal{G}$, and in which each cell possess its own "internal" symmetry group $\mathcal{L}$, where $\mathcal{G}$ is a subgroup of the permutation group $\mathrm{S}_{N}$ of $N$ elements and $\mathcal{L}$ is a compact Lie group. The coupling we consider is invariant under the internal symmetries of each cell and the combination of the groups $\mathcal{L}$ and $\mathcal{G}$ leads to the total symuctry group given by $\mathcal{L}$ wreath product $\mathcal{G}, \mathcal{L}$ iG, i. e., $\mathcal{L}^{N}+\mathcal{G}$. We rolate the steady-state bifurcations that occur in the coupled system with symmetry group $\mathcal{L}$ ? $\mathcal{G}$ to bifurcations with symmetry $\mathcal{L}$ or $\mathcal{G}$. We apply the results to a non-degenerate system of $N$ coupled cells with symmetry $\mathrm{O}(2)<\mathrm{S}_{N}$. We see how the invariant theory for $\mathrm{O}(2)<\mathrm{S}_{N}$ is related to the invariant theories for $O(2)$ and $S_{N}$. We check that, up to conjugacy, there are exactly $N$ branches, namely, those with axial subgroup. Moreover, we discuss stability and directions of the solution branches.
\end{abstract}




\title{
Sumário
}

\author{
Introdução
}

1 Preliminares teóricas 1

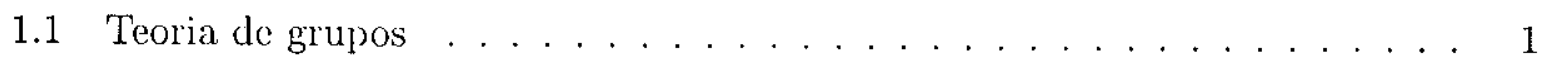

1.1 .1 Grupos de Lic . . . . . . . . . . . . . . . . . . 1

1.1 .2 Representação c ação . . . . . . . . . . . . . . . . . 4

1.1 .3 Integração invariante . . . . . . . . . . . . . . . . 7

1.2 Irredutibilidade . . . . . . . . . . . . . . . . . . 8

1.2 .1 Definições básicas e excmplos . . . . . . . . . . . . 8

1.2 .2 Decomposição isotipica . . . . . . . . . . . . . . . . 10

1.3 Teoria básica de bifurcạ̧ão equivariante . . . . . . . . . . . . . . . 14

1.3.1 Os conceitos de germe e bifurcação . . . . . . . . . . . . . 14

1.3.2 Funçôes invariantes . . . . . . . . . . . . . . . . . . . . . . . . . . . . . 15

1.3.3 Aplicações lincares equivariantes . . . . . . . . . . . . . . . . . . 19

1.3.4 Aplicações não lineares cquivariantes . . . . . . . . . . . . . . . . 21

1.4 Quebra de simetria cm bifurcação de pontos de equilíbrio . . . . . . . . . 23

1.4.1 Órbitas c subgrupos de isotropia . . . . . . . . . . . . . . 23

1.4.2 Subespaço de ponto fixo . . . . . . . . . . . . . . 26

1.4.3 Lema dos Ramos Equivariantes . . . . . . . . . . . . . . . . . 28

1.4.4 Estabilidade de soluções . . . . . . . . . . . . . . . 31

2 Sistemas de células acopladas com simetria do tipo produto coroa $\quad 34$

2.1 EDOs de sistemas acoplados com simetria do tipo produto coroa . . . . . . 35

2.2 Teoria linear do grupo $\mathcal{L} \imath \mathcal{G} \ldots \ldots \ldots$. . . . . . . . . . . . . . . 37

2.3 Quebra de simetria cm bifurcação $\mathcal{L}$ 々 $\mathcal{G}$-cquivariante . . . . . . . . . . . . . 39

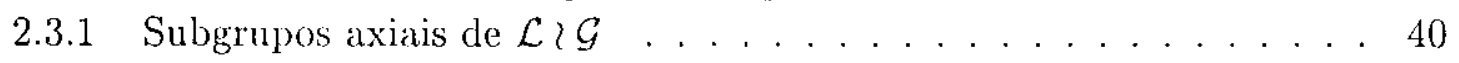

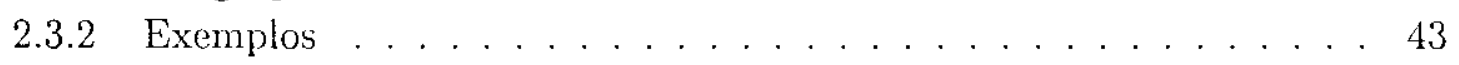

2.3.3 Subgrupos de isotropia e subgrupos de isotropia maximais de $\mathcal{L}$ ₹ $\mathcal{G} \quad 46$

3 Bifurcação de pontos de equilíbrio com simetria $\mathrm{O}(2)$ ? $\mathrm{S}_{N} \quad 49$

3.1 Teoria invariante o equivariante para o grupo

$\mathbf{O}(2)<\mathcal{G} \ldots \ldots \ldots \ldots \ldots \ldots$ 
3.2 Teoria invariante e equivariante para o grupo $\mathbf{O}(2) \geq \mathbf{S}_{N} \ldots \ldots \ldots \ldots \ldots \ldots \ldots$

3.3 Diagramas de bifurcaça e estabilidade de solucoes . . . . . . . . . . 60

A Redução de Liapunov-Schmidt $\quad 74$

A.1 A Redução de Liapunov-Schmidt com simetria . . . . . . . . . . . . . . . 77

$\begin{array}{ll}\text { Referências Bibliográficas } & 81\end{array}$ 


\section{Lista de Figuras}

1.1 Polígono regular de 5 lados . . . . . . . . . . . . . . . . . . . . . 24

1.2 Ramos de soluşões (a) subcrítico e (b) supercrítico . . . . . . . . . . . . 31

2.1 Diagramas da cólulas ativas/inativas em um anel de 15 cólulas idênticas com simetria interna $\mathbf{O}(2) \ldots \ldots \ldots \ldots 4 . \ldots \ldots$

2.2 Diagramas da células ativas/inativas en um anel de 12 cólulas idênticas com simetria interna $\mathbf{O}(2) \ldots \ldots \ldots \ldots$. . . . . . . . . . . . 47

3.1 a) Acoplamento de 4 células duas a duas: $\mathcal{G}=\mathbf{S}_{4}$, b) Acoplamento de 5 cólulas duas a duas: $\mathcal{G}=\mathrm{s}_{5} \ldots \ldots \ldots \ldots \ldots$

3.2 Varicdade de transição . . . . . . . . . . . . . . . . . . 70

3.3 Diagrama para $(a, b) \in D_{j}, 1 \leq j<N \ldots \ldots \ldots \ldots$

3.4 Diagrama para $(a, b) \in A_{j}, 1 \leq j \leq N \ldots \ldots \ldots \ldots$

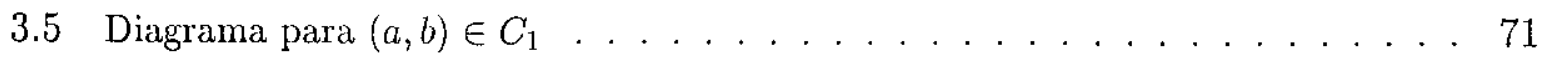

3.6 Diagrama para $(a, b) \in C_{N} \ldots \ldots \ldots \ldots \ldots \ldots$ 


\section{Lista de Tabelas}

1.1 Tipos de órbitas e subgrupos de isotropia do grupo $\mathbf{D}_{n}$ agindo em $\mathbb{C}$ com $n$ ímpar . . . . . . . . . . . . . . . . . . . . 25

2.1 Subgrupos axiais de $\mathrm{O}(2) \backslash \mathrm{D}_{15}$ a monos de conjugação . . . . . . . . 43

2.2 Subgrupos de $\mathrm{D}_{12} \ldots \ldots \ldots \ldots \ldots \ldots$

2.3 Subgrupos axiais do $\mathrm{O}(2) \geq \mathrm{D}_{12} \ldots \ldots \ldots \ldots$

3.1 Decomposição de $\mathbb{C}^{N}$ cm subespaços invariantes por $\Sigma\left(\mathbb{Z}_{2}^{\kappa},\{1, \ldots, j\}\right), 1 \leq$

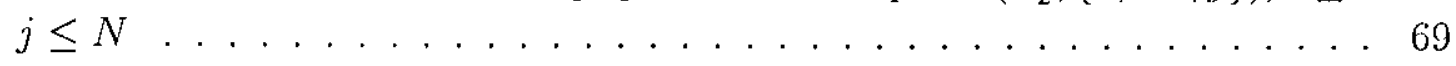

3.2 Ramos e estabilidade de soluções do problema de bifurcação com simetria $\mathbf{O}(2)$ ? $\mathbf{S}_{N}$ relativamente aos seus subgrupos de isotropia . . . . . . . . . 69 


\section{Introdução}

Nos últimos vinte e cinco anos, a presença de simetria em sistemas dinâmicos tem sido foco de atenção de vários autores. Em bifurcação de pontos de equilíbrio, simetrias impõem fortes restrições na forma das soluçōes e na maneira pela qual as bifurcações ocorrem. O termo bifurcação se refere ao fenômeno de aparecimento de novas soluções de equilíbrio num sistema além da solução trivial com a variação do parâmetro de bifurcação. Iniciamos este trabalho apresentando a teoria geral de bifurcaçōes de pontos de equilíbrio no contexto com simetria seguindo [10]. Mais precisamente, considerando um grupo de Lie compacto $\Gamma$ agindo em um espaço vetorial $V$, cstudamos pontos de equilíbrio de um sistema do tipo

$$
\dot{x}+g(x, \lambda)=0
$$

com a variação do parâmetro $\lambda \in \mathbb{R}$, onde $g: V \times \mathbb{R} \rightarrow V$ ć uma aplicação $\Gamma$-equivariante (ou com grupo de simetrial l'), isto é,

$$
\gamma g(x, \lambda)=g(\gamma x, \lambda), \quad \forall x \in V, \forall \gamma \in \Gamma .
$$

Neste contexto, duas definições básicas são as de um subgrupo de isotropia de um elemento $x \in V$ e a de um subespaço de ponto fixo de um subgrupo $\Sigma$ : Dado $x \in V$, o subgrupo de isotropia de $x$, denotado por $\Sigma_{x}$, é delinido como

$$
\Sigma_{x}=\{\sigma \in \Gamma ; \sigma x=x\} \text {. }
$$

O subespaço de ponto fixo de $\Sigma$, denotado por $F i x \Sigma$, é definido como

$$
F i x(\Sigma)=\{v \in V: \sigma v=v, \forall \sigma \in \Sigma\}
$$

No contexto com simetria a questão fundamental é: Para $g$ como em (0.0.2), para quais subgrupos $\Sigma$ de $\Gamma$ devenos esperar encontrar ramos de soluções de equilíbrio de (0.0.1) tendo $\Sigma$ como seu grupo de simetrias? Pensamos no grupo total de simetria $\Gamma$ da solução trivial se quebrando para o subgrupo $\Sigma$ de simetrias de um novo ramo de soluções não triviais. Em outras palavras, ganliamos novas soluçós embora com menos sinetria que as originais. Este processo é chamado quebra espontânca de simetria. O Lema dos Ramos Equivariantes responde a questão acima, garantindo quc, genericamente, para cada subgrupo de isotropia $\Sigma$ com dim Fix $\Sigma=1$, chanados subgrupos axiais, existe un único ramo de soluções tal que o grupo de simetria de cada solução neste ramo é $\Sigma$. 
Mais recentemente, ten havido um aumento de interesse no estudo de bifurcações em sistemas de "cólulas" acopladas, por exemplo, un conjunto de osciladores acoplados. O objetivo deste trabalino é estudar bifurcação de pontos de exuilíbrio om tais sistemas no contexto com simetria. Em geral, uma célula cm tais sistemas é representada por uma cquação difercncial $x^{\prime}=f(x)$, definida num espaço vetorial $V$ de dimensão finita, com a hipótese de existência de um ponto de equilíbrio, por excmplo $x=0$, ou seja, $f(0)=0$. Um sistema de $N$ cólulas identicas acopladas é un sistema de equações diferenciais en $V^{N}$ da forma

$$
x_{j}^{\prime}=f\left(x_{j}\right)+g_{j}\left(x_{1}, \ldots, x_{N}\right), \quad 1 \leq j \leq N,
$$

com $y_{j}(x)=0$ se $x_{i}=0$ para $i \neq j$ e $g_{j}(x)=0$, se $x_{j}=0$. As funções $g_{j}$ 's dão 0 acoplamento entre a cólula $j$ com as demais cólulas clos sistema.

Uma forma de construir um sistema de cólulas acopladas e que tem sido tratada recentemente por vários autores ć assumir simetrias "internas" (para as células individuais) e impor restriçõos de simetrias "globais" nas funçōes de acoplannonto. Vamos descrever em mais detalhes a seguir: Quando tais células sāo idênticas, as simetrias globais induzidas no sistema de equações diferenciais associado [4] dependem da forma exata de acoplamento. Por exemplo, um sistema de $N$ células acopladas num ancl [9] possui simetria do grupo diedral $\mathbf{D}_{N}$, grupo de simetrias do polígono regular de $N$ lados. Outro padrão é o acoplannento do tipo duas a duas, onde cada cólula é acoplada a qualquer outra do sistema, induzinclo neste sistena as simetrias do grupo $\mathrm{S}_{N}$, grupo clas pernutaçós de $N$ elementos. Estas simetrias induzidas pelo padrão de acoplamento são as chamadas simetrias globais do problema. O grupo das simetrias globais é sempre um subgrupo $\mathcal{G}$ de $\mathrm{S}_{N}$. Um outro conjunto de simetrias para as células acopladas tem sido considerado mais recentemente; ele ocorrc quando as equações diferenciais que governam a dinâmica cm cada célula têm suas próprias simetrias. Isto ocorre, por exemplo, quando cada célula é vista como um objeto geométrico com certas simetrias - como um disco circular - c a dinâmica em cada célula é governada por equações diferenciais invariantes por estas simctrias. Um outro exemplo comum é um conjunto de osciladores de Van der Pol acoplados, cada um dos quais possui a simetria da reflexão $(x, y) \mapsto(-x, y)$, onde $(x, y)$ são as variáveis de estado de um dos osciladores. Estas simetrias são chamadas simetrias internas do sistema, que denotamos abaixo por $\mathcal{L}$.

Quando tais sistemas dependem de un parâmetro, a forma como pontos de equilíbrio bifurcam com a variação deste parâmetro depende tanto das sinctrias globais quanto das simetrias internas. Ln fato sutil nesta discussão ć que o grupo total de simetrias do problema depende da forma exata do acoplamento. Embora em qualquer sistema acoplado o grupo é obtido a partir de $\mathcal{G}$ c $\mathcal{L}$, a forma como estes grupos combinam também depende da forma de acoplamento. Existem dois tipos naturais de acoplamento que levan a dois grupos distintos. En um deles, o acoplannento é invariante segundo qualquer simetria interna de cada célula e consiste do grupo "produto coroa" $\mathcal{L}<\mathcal{G}$. No segundo tipo, as simetrias internas deven ser aplicadas a todas as células simultancanente consiste, neste caso, do produto direto $\mathcal{L} \times \mathcal{G}$. Neste projeto tratamos do princiro tipo de acoplamento. 
Relacionamos neste trabaltho as bifurcações de pontos de equilíbrio que ocorrem em sistemas acoplados com grupo de simetria $\mathcal{L} \imath \mathcal{G}$ às bifurcacoes de problemas $\mathcal{L}$-cquivariantes e problemas $\mathcal{G}$-equivariantes seguindo os resultados obtidos em [4]. Esta correspondencia organiza o problena, reduzindoro a questốes mais simples cujas respostas săo obtidas de resultados conhecidos. Fiazenos und aplicaça ao estado de existencia e estabilidade de pontos de equilíbrio para um problema particular de osciladores acoplados com simetria do grupo $\mathrm{O}(2)<\mathrm{S}_{N}$.

No Capítulo 1, tratamos da teoria de grupos. Introduzimos a idéia de um grupo de Lie compacto $\Gamma$, noģós e propriedades de ação e representaçio de $\Gamma$ num espaço vetorial $V$. Descrevemos exemplos fundamentais como o grupo ortogonal $\mathrm{O}(N)$, o grupo do circulo $\mathrm{S}^{\natural}$, o grupo diedral $\mathbf{D}_{N}$, o grupo das permutações $\mathbf{S}_{N}$ e o grupo "produto coroa" juntamente com suas ações. Com a definiģão de integral de Haar mostramos que podemos identificar um grupo de Lie compacto $\mathrm{l}^{\prime}$ com um subgrupo de $\mathrm{O}(N)$. Descrevemos a decomposição de urna dada represcntação en representaçôes mais simples, chamadas representações irredutíveis. Neste capítulo, discutinos a teoria básica de bifurcação equivariante e tratamos das noções de uma aplicação $g: V \times \mathbb{R} \rightarrow V$ I'-çuivariante, uma função $\int: V \times \mathbb{R} \rightarrow \mathbb{R}$ $\Gamma$-invariante, subgrupos de isotropia e bifurcações com quebra espontânea de simetria. Apresentamos também um dos resultados mais inportantes antes desta teoria, o Lema dos Ramos Equivariantes. Para finalizar o capítulo, discutimos estabilidade de pontos de equilíbrio em sistemas equivariantes.

No Capítulo 2, apresentamos resultados sobre o tema principal, isto é, bifurcação de pontos de equilíbrio en sistemas de equações diferenciais ordinárias de $N$ células idênticas acopladas. Consideramos este sistema com presença de simetria global $\mathcal{G}$ e interna $\mathcal{L}$, com - acoplamento invariante segundo elementos de $\mathcal{L}$ e que leva à simetria do tipo produto coroa de $\mathcal{L}$ e $\mathcal{G}, \mathcal{L}$ < $\mathcal{G}$. Caracterizamos os subgrupos de isotropia de $\mathcal{L}$ $\mathcal{G}$ e descrevemos os subgrupos axiais e maximais. Os resultados obtidos foram aplicados aos casos das ações de $\mathrm{O}(2)<\mathrm{D}_{15}$ e $\mathrm{O}(2)<\mathrm{D}_{\downarrow 2}$ nlo plano.

No Capítulo 3, fazomos una aplicação da teoria apresentada nos capítulos anteriores para estudar bifurcações de pontos de equilíbrio para um sistema de $N$ cólulas idênticas acopladas com simetria $\mathrm{O}(2) \geq \mathrm{S}_{N} \mathrm{~cm} V \ldots \mathbb{C}^{N}$ onde $V$ a umi representaçào $\mathrm{O}(2)$ \& $\mathrm{S}_{N^{-}}$ absolutamente irredutivel. Obtemos a forma geral cle un campo vetorial $g: V \times \mathbb{R} \rightarrow V$ $\mathrm{O}(2) ? \mathrm{~S}_{N}$-equivariante a um parâmetro de bifurcação. Mostramos como a teoria invariante para $\mathrm{O}(2)<\mathrm{S}_{N}$ está relacionada às teorias invariantes para os grupos $\mathrm{O}(2)$ e $\mathcal{G}$, onde $\mathcal{G}$ é um subgrupo de $\mathrm{S}_{N}$. Descrevemos os ramos de soluções $(x, \lambda)$ para $g(x, \lambda)=0$ vindos do Lema dos Ramos Equivariantes e verificamos que, a menos de conjugação, existem exatamente $N$ desses ranus.

Finalmente, no Apêndice A desenvolvemos a Redução de Liapunov-Schimidt em sistemas definidos em espaços de climensão infinita quando a parte linear do operador tem uúcleo de dimensão finilia. Esta redução cstabelece uma correspondencia local entre as soluções do sistema original com as soluções do sistema reduzido. Além clisiso, no casso cm que o sistema original possui simetria mostramos que o sistema reduzido pela Reduf̧ão 
de Liapunov-Schimidt preserva esta simetria. 


\section{Capítulo 1}

\section{Preliminares teóricas}

A idéia principal deste trabalho envolve bifurcasão de pontos de equilíbrio en sistemas com simetria. Una simetria de um sistema definido num conjunto $\mathcal{X}$ ć una transformação de $\mathcal{X}$ que preserva algumas estruturas particulares deste sistema. Nos nossos estudos $\mathcal{X}$ ć um espaço vetorial de dimensão finita, as transformargốcs são aplicações lineares em $\mathcal{X}$, c a estrutura a ser preservada ć un problema de bifurcação particular.

\subsection{Teoria de grupos}

No cstudo de problenas com sinctria, a teoria de grupos é indispensável. A seguir apresentamos alguns fatos sobre grupos de Lic.

\subsubsection{Grupos de Lie}

Para definirmos grupo de Lie apresentamos algumas definições.

Definições 1.1.1 Una parametrização $n$-dimensional de um conjunto $X \subseteq \mathbb{R}^{j}$ é uma aplicą̧ăo suave $\left(\mathbb{C}^{\infty}\right) \phi: V \rightarrow \mathbb{R}^{j} \operatorname{com} V \subseteq \mathbb{R}^{n}$ aberto tal que $\phi(V)=X$ e a aplicação $\phi: V \rightarrow X$ é um difcomorfismo, ou seja, $\phi: V \rightarrow X$ é uma bijcção c $\phi c \phi^{-1}$ são suaves.

Suponhamos $Y \subseteq \mathbb{R}^{k}$ c $x \in Y$. Uma vizinhança relativamente aberta de $x$ em $Y$ é um conjunto da forma $U \cap Y$ com $U \subseteq \mathbb{R}^{k}$ um conjunlo aberto com $x \in U$.

Chamamos $N \subseteq \mathbb{R}^{k}$ uma variedade suave de dimensão $n$ quando todo ponto $x \in N$ tem uma vizinhança relativamente aberta $U \cap Y$ admitindo uma parametrizaşão $n$-dimensional $\phi: V \rightarrow \mathbb{R}^{k}$.

Agora introduzinos a nossa definição de maior interesse.

Definição 1.1.2 Un grupo de Lie é um grupo $G$ que é uma variedade suave tal que as operaçöes de multiplicas̆ón $G \times G \rightarrow G$ e inversäo $G \rightarrow G$ são aplicaşós suaves. 
O grupo linear GL $(n)$ das matrizes $n \times n$ inversíveis é um grupo de Lie. De fato, como o grupo GL $(n)$ é un subconjunto aborto do espaço dats matrizes $n \times n$, entáo $\mathrm{GL}(n)$ é uma variedade suave. Agora, multiplicarão de matrizes $n \times n$ é uma aplicarão polinomial, e certamente suave, a assim a restriçăo da multiplieaşão ao grupo GL(n) também é suave. Como todas as matrizes de GL(n) possuem determinante diferente de zero, cntão todas têm inversa e assim a aplicação inversa também é suave.

Definição 1.1.3 Grupo de Lie lincar ém subyrupo $\mathrm{l}^{\prime} \subset \mathrm{GL}(n)$ fechado.

Nos nossos estudos precisimos que $\Gamma$ seja $u m$ grupo de Lie. Para nossos propósitos, consideramos um grupo de Lie como um subgrupo fechade $\Gamma$ de $G L(n)$, que pela Definição 1.1.3 estes säo os grupos de Lie lincir e para o termo grupo de Lie é dada una definiçá mais geral, Definição 1.1.2. Entretanto, existe un teorena (ver Bourbaki [1960]) que afirma que todo grupo de Lie compacto no sentido geral e topologicamente isomorfo a um grupo de Lie linear. Identificando o espaço das matrizes $n \times n$ com o espaço topológico $\mathbb{R}^{n^{2}}$ podemos falar das propriedades topológicas do grupo de Lie. Lim particular, dizemos que um grupo de Lic $\Gamma$ é un subconjunto fechado de $G L(n)$ e também um subgrupo.

As vezes nos referimos a certos grupos como grupos de Lie, mas que na verdade são subgrupos isomorfos a algum subgrupo do $\mathrm{GL}(n)$. Por cxemplo, nos referimos ao grupo com dois elementos $\mathbb{Z}_{2}=\{1,-1\}$ como sendo un grupo de Lie e a maneira mais correta seria $\mathbb{Z}_{2}=\{1,-1\}$ isonorfo ao grupo de Lie $\left\{I_{n},-I_{n}\right\} \subset \operatorname{GL}(n)$, onde $I_{n}$ ó a matriz identidade $n \times n$.

Apresentamos a seguir alguns exemplos de grupos de Lie.

Exemplos 1.1.4 1. O grupo ortogonal $\mathrm{O}(n)$ consiste de todas as matrizes, $A, n \times n$ tal que

$$
A A^{l}=I_{n}
$$

onde $A^{t}$ é a malriz transposta de $A$, isto é,

$$
\mathrm{O}(n)=\left\{A \in \mathrm{GL}(n) ; A A^{\prime}=I_{n}\right\} .
$$

2. O grupo cspecial ortogonal $\mathrm{SO}(n)$ consiste de hodas as matrizes $A \in \mathrm{O}(n)$ lal que det $A=1$ :

$$
\mathrm{SO}(n)=\{A \in \mathrm{O}(n) ; \operatorname{det} A=1\} \subset \mathrm{O}(n) \subset \mathrm{GL}(n) .
$$

Este grupo é chamado de grupo das rotaçòes em. $\mathrm{X}^{n}$. Em particular, $\mathrm{SO}(2)$ consiste das rotaçoes no phano

$$
\Gamma_{0} \cdots\left(\begin{array}{cc}
\cos \theta & -\operatorname{sen} \theta \\
\operatorname{sen} \theta & \cos \theta
\end{array}\right)
$$

Desta mancira, podemos identificar $\mathrm{SO}(2)$ com o grupo cáclico $\mathrm{S}^{1}$, onde a identificaçäo é dada pola aplicaşào: 


$$
R_{\theta}=\left(\begin{array}{cc}
\cos \theta & -\operatorname{sen} \theta \\
\operatorname{sen} \theta & \cos \theta
\end{array}\right) \mapsto \theta
$$

O grupo $\mathrm{O}(2)$ é gerado por $\mathrm{SO}(2)$ junlamente com a reflexão

$$
\kappa=\left(\begin{array}{cc}
1 & 0 \\
0 & -1
\end{array}\right)
$$

3. Seja $\mathbb{Z}_{n}$ o grupo cíclico de ordem n. Podemos identificur $\mathbb{Z}_{n}$ com o grupo das matrizes de rotação $2 \times 2$ geradas por $R_{2 \pi / n}$.

4. O grupo diedral $\mathbf{D}_{i 2}$ de ordem $2 n$ é gerado pelo grupo $\mathbb{Z}_{n}$ e um elemenlo de ordem 2

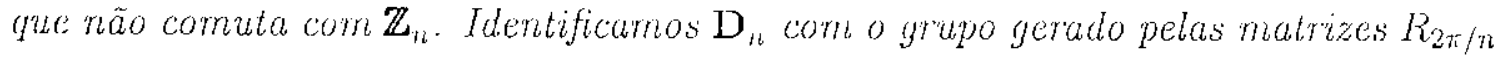
e pela reflexão de (1.1.2). Geonetricanenle, $\mathrm{D}_{n}$ a grupo de simetrias do polígono regular de $n$ lados, enquanto $\mathbb{Z}_{n}$ é o subgrupo das rolaşós simétricas.

5. Todo grupo finito é isomorfo a um grupo de Lie.

6. O toro de dimensão $n, \mathrm{~T}^{n}=\underbrace{\mathrm{S}^{\mathrm{i}} \times \mathrm{S}^{1} \times \ldots \times \mathrm{S}^{1}}_{n-v c i c s}$, é isomorfo a um grupo de Lie, com o isomorfismo que leva $\theta=\left(0_{1}, \cdots, 0_{n}\right) \in \mathrm{T}^{n}$ à malriz

$$
\left(\begin{array}{cccc}
R_{\theta_{1}} & 0 & \cdots & 0 \\
0 & R_{\theta_{2}} & \cdots & 0 \\
\vdots & \vdots & \ddots & \vdots \\
0 & 0 & \cdots & R_{\theta_{n}}
\end{array}\right) \in G L(2 n)
$$

7. $\mathbb{R}^{n}$ é isomorfo ao grupo de Lie das matrizes da forma

$$
\left(\begin{array}{ccccc}
1 & a_{1} & a_{2} & \cdots & a_{n} \\
0 & 1 & 0 & \cdots & 0 \\
0 & 0 & 1 & \cdots & 0 \\
\vdots & \vdots & \vdots & \ddots & \vdots \\
0 & 0 & 0 & \cdots & 1
\end{array}\right) \in G L(n+1)
$$

onde $a_{i} \in \mathbb{R}$, para $i=1, \ldots, n$.

Vimos que um dado grupo abstrato pode ser representado de várias maneiras como grupo de matrizes. A questão que surge é: quanclo dois grupos de matrizes que são isomorfos como subgrupos abstratos são considerados essencialnente os mesmos? Esta questão leva diretarnete à teoria de representação a seguir.

Temos que $\mathbb{R}^{n^{2}}$ é um espago topológico, logo podemos falar das propriedades topológicas bem como das algébricas de um grupo de Lie. En particular, dizomos que um grupo de Lie $\Gamma$ é compacto ou conexo se ele é compacto ou concxo como subconjunto de $\mathbb{R}^{n^{2}}$. Daí, $\Gamma$ ć um grupo de Lie compacto se, e somente se, as entradas das matrizes que definem $\Gamma$ são limitadas, isto porque já temos, por defuigão, quo $\Gamma$ é fechado. Segure que $O(n)$, $\mathrm{SO}(n), \mathrm{T}^{n}$ e os grupos finitos são exemplos de grupos de Lie compactos. 


\subsubsection{Representação e ação}

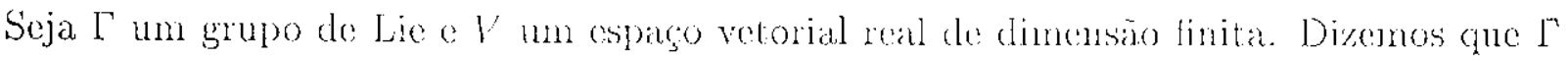
age linearmente on $V$ se cxiste ma aplicaça continua, a ação do $\Gamma \mathrm{em} V$,

$$
\begin{aligned}
& \Gamma \times Y \rightarrow V \\
& (\gamma \cdot v) \rightarrow \gamma \cdot v
\end{aligned}
$$

tal que

a) para cada $\gamma \in \Gamma$ a aplicação

$$
\begin{aligned}
\rho_{\gamma}: V & \rightarrow V \\
v & \mapsto \rho_{\gamma}(v)=\gamma \cdot v
\end{aligned}
$$

ć lincar

b) se $\gamma_{1}, \gamma_{2} \in \Gamma$ entiono

$$
\gamma_{1} \cdot\left(\gamma_{2} \cdot v\right)-\left(\gamma_{2} \gamma_{2}\right) \cdot v
$$

A aplicação $\rho$ que leva $\gamma$ en $\rho_{\gamma} \in \mathrm{GL}(V)$ é chanada una representação de $\Gamma$ cn $V$, onde $\mathrm{GL}(V)$ denota o conjunto des's transformaroes lincares invertiveis de $V$ em $V$. Nas próximas seçós omitimos o ponto da operacão escrevendo $\gamma$ u no lughr de $\gamma \cdot v$. Como exemplo, temos a aräo do grupo cíclico $\mathrm{S}^{1}$ no espaco vetorial $\mathbb{C} \equiv \mathbb{B}^{2}$ dada por

$$
\begin{aligned}
& \mathrm{S}^{1} \times \mathbb{C} \\
& (0, z) \rightarrow 0 \cdot z=e^{i \theta} z .
\end{aligned}
$$

Neste exemplo, a lincaridale de (1.1.4) segue inediatanente da defindäo (1.1.6). Vanos verificar que vale (1.1.5), Para $\theta_{1}, \theta_{2} \subset \mathrm{S}^{\prime}$

$$
\theta_{1} \cdot\left(\theta_{2} \cdot z\right)-\theta_{1} \cdot\left(e^{i \theta_{2}} z\right)=e^{i i_{1}} e^{i \theta_{2}} z=e^{i\left(\theta_{1} \mid \theta_{2}\right)} z=\left(\theta_{1}+\theta_{2}\right) \cdot z,
$$

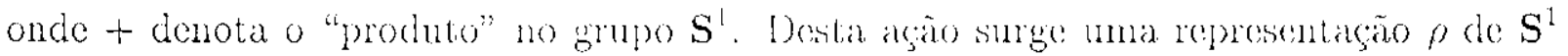
onde $\rho_{0}$ é a matriz de rotagăo

$$
\left(\begin{array}{cc}
\cos \theta & -\operatorname{sen} \theta \\
\operatorname{sen} \theta & \cos \theta
\end{array}\right) .
$$

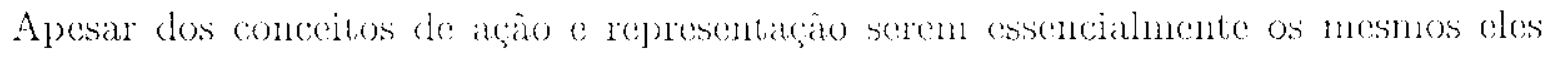
possuem uma pequena diferenca no ponto de vista, on seja, wana aço diz como un clemento $\gamma$ do grupo transforna un dado elenento $\theta E V$, encpuanto que una representação

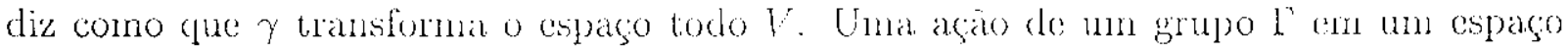
vetorial $V$ pode ser defuida especificando-se (1.1.3) somente para os geradores de $\Gamma$. Fsta ação é consistente no sentido de que (1.1.5) estáa satisfeita. 


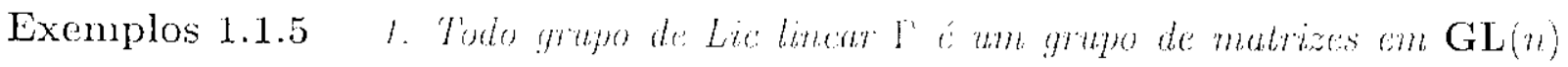

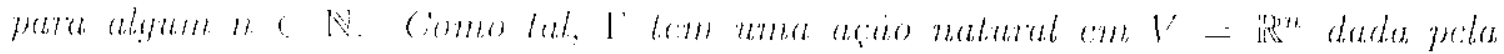

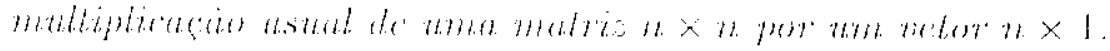

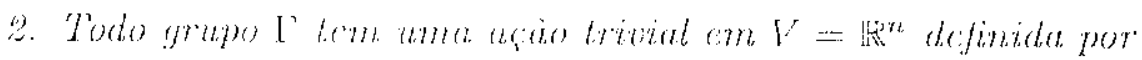

$$
\begin{aligned}
& \Gamma \times \mathbb{R}^{n-\rightarrow} \quad \mathbb{R}^{\prime \prime} \\
& (\because, x) 1>\quad \cdot n \cdots x
\end{aligned}
$$

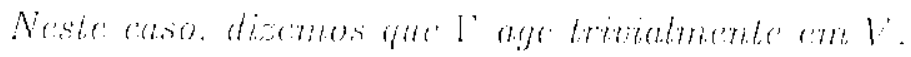

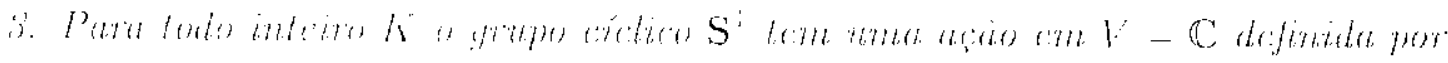

$$
\begin{aligned}
& S \times \mathbb{C} \rightarrow \mathbb{C} \\
& (0,2) \rightarrow 0 \cdot z-0^{n+\infty} z .
\end{aligned}
$$

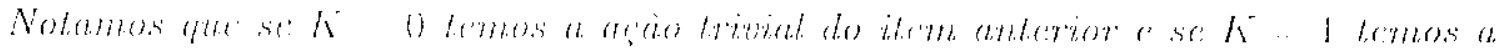
thsio (1.1.0\%).

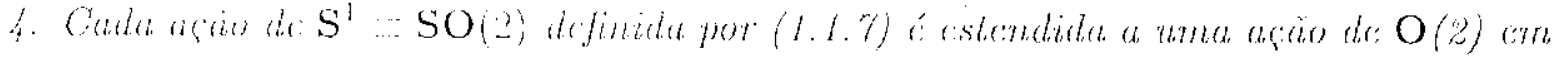
C se constertemes

$$
\lambda \cdot z=z
$$

onderis a reflento (1.1.2).

5. Todo grupo de Lie $\Gamma \subset \mathrm{GL}(n)$ age no espaco das matrizes $n \times$ n por semellança

$$
\because \cdot A \cdots=\gamma+\cdots !
$$

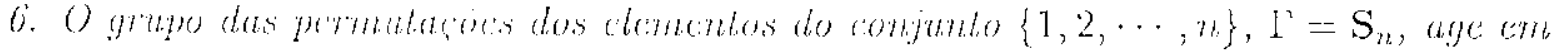
$\mathbb{R}^{\prime \prime}$ pelu urion

$$
\sigma \cdot\left(x_{1}, x_{2}, \cdots, x_{n}\right)=\left(x_{0},\left(1, x_{0}-1(2), \cdots, x_{\sigma} \cdots,(n)\right)\right.
$$

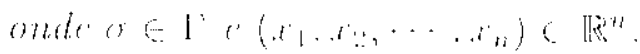

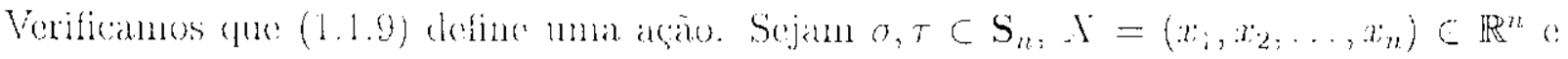
$Y^{*}-\tau \cdot X=\left(x_{T} 1(1), x_{i} \cdots(2) ; \cdots, n_{T} \cdot{ }_{(n)}\right) \subset \mathbb{R}^{\prime \prime}$.

$$
\begin{aligned}
& \sigma \cdot(\tau \cdot \mathrm{Y})=\sigma \cdot \mathrm{Y}^{\gamma}=\left(y_{\sigma} \cdots+(1), y_{t}(2): \cdots, y_{\sigma}:(n)\right)^{--}
\end{aligned}
$$

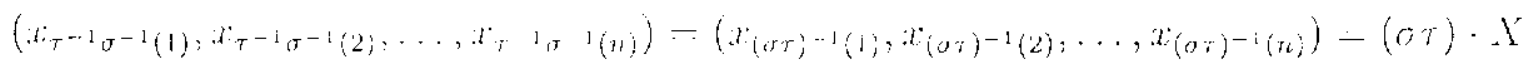

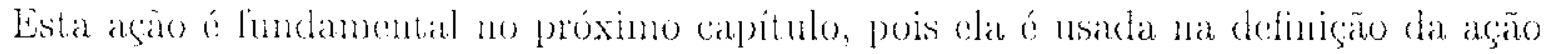
do produlo coroar. 
Definiçāo 1.1.6 Sejam $V$ eW espaços veloniais de dimbensăo n e $\Gamma$ um grupo de Lie

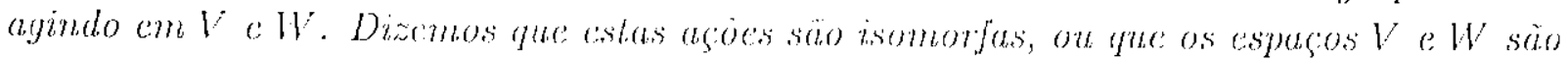
I'isomorfos, se eniste um isomorfismo linear $A: V \rightarrow W$ que comula com lodo clemento de $\Gamma$, isto é,

$$
A(\gamma \cdot v)-\gamma \cdot(1 v)
$$

para lodo $v \in V$ e $\gamma \in \Gamma$.

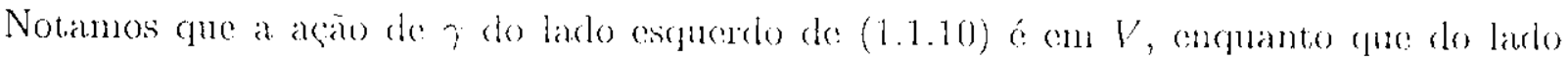
direito é en $W^{r}$.

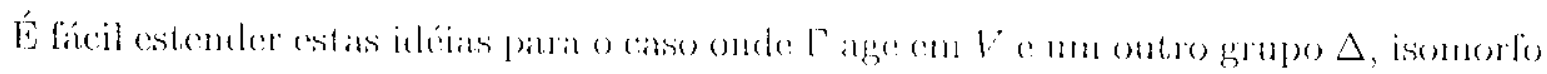

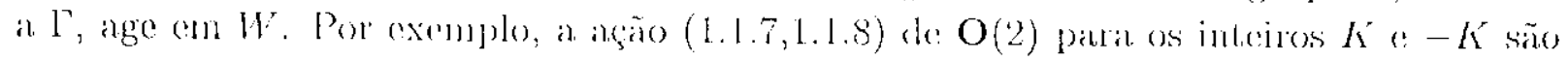

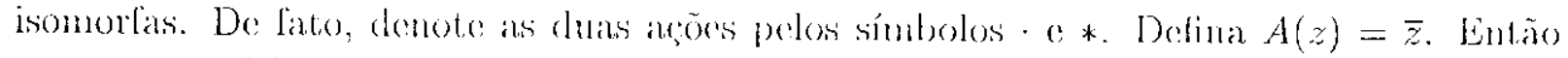
para $\gamma \in \mathrm{SO}(2)$ e r a relloxio (1.1.2) lemos:

$$
A(\gamma \cdot z)=A\left(e^{i \theta \kappa} z\right)=\overline{c^{i K i} z}=c^{-i \kappa \theta} \bar{z}=e^{-i \kappa \theta}(A(z))=\gamma *(A(z))
$$

c

$$
A(k \cdot z)=A(z)=\bar{z}-z=\mu * z-\mu * A(z) .
$$

Da mesma manciral, os grupos $\mathrm{SO}(2)$ o $\mathrm{S}^{\prime}$ säo isomorlos a a ação (1.1.7) do $\mathrm{S}^{b}$ an

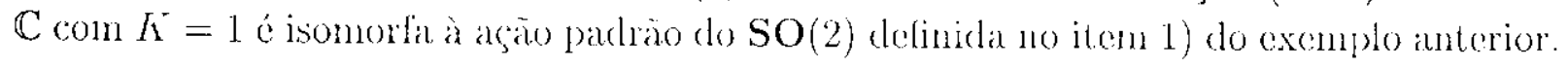

Definimos a seguir o principal grupo do nosso traballio, o grupo produto corora e a sua ã̧üo em $V^{n}$, onde $V=\mathbb{R}^{k}$ para algum $k \in \mathbb{N}$.

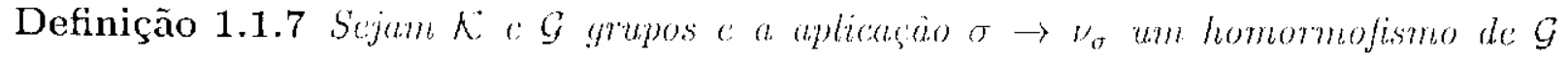
no grupo dos automorfismos $A(\mathcal{K})$ de $\mathcal{K}$. Entáo o conjunto de todos os pares ordenados $(k, \sigma), k \in \mathcal{K}$ e $\sigma \in \mathcal{G}$, forma um grupo, o produto semi-direto de $\mathcal{K}$ e $\mathcal{G}$, denotado por $\mathcal{K}+\mathcal{G}$, com a operaços de mulliplicascan

$$
(k, \sigma)\left(k^{\prime}, \sigma^{\prime}\right)-\left(k \nu_{\sigma}\left(k^{\prime}\right), \sigma \sigma^{\prime}\right) .
$$

Definição 1.1 .8 Dalos um grupo $\mathcal{L}$ e um subgrupo $\mathcal{G}$ do grupo $\mathrm{S}_{n}$ das permulaçoes, o produto coroa de $\mathcal{L}$ e $\mathcal{G}$ é o produto semi-direto de $\mathcal{L}^{n}$ e $\mathcal{G}$, denotado por $\mathcal{L}$ i $\mathcal{G}$.

Observação 1.1 .9 () protuluo coroa $\mathcal{L}$ ? $\mathcal{G}$ c unt grupo gerado por $\mathcal{L}^{u}$ o $\mathcal{G}$. A seguir apresentamos a cstrutura do grupo produto coroa e sua aça em $V^{n}$.

Seja $V=\mathbb{R}^{k}$. A acjio do grupo produto coroa $\mathcal{L}<\mathcal{G}$ on $V^{n}$ é dado por:

$$
(l, \sigma) \cdot\left(x_{1}, x_{2}, \cdots, x_{n}\right)=\left(l_{1} x_{\sigma} \text { in } u_{1}, l_{2} x_{0}-1(2), \cdots, l_{n} x_{\sigma^{-1}(n)}\right)
$$

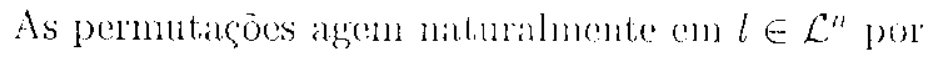

$$
\sigma \cdot l=\left(l_{i},(1), \cdots, l_{n, 1(n)}\right) \text {. }
$$

Estes são os antomorfismos da Deliniçio 1.1.7. O grupo produto coroa é de fato un grupo com a operaçäo

$$
(h, \tau)(l, \sigma)=(h, \tau(l), \tau \sigma)
$$




\subsubsection{Integração invariante}

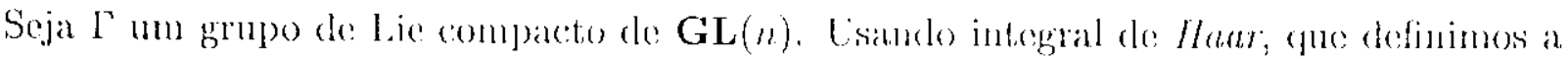
seguir, identificamos $\Gamma$ com um subgrupo ortogonal do grupo $\mathrm{O}(n)$. Este tipo de integragào é invariante sob translação por clementos de $\Gamma$.

Definição 1.1.10 Dada $f: \Gamma \rightarrow \mathbb{R}$ uma funçäo contúnua a valores reais, a opreração

$$
\int_{\gamma \in \Gamma^{\Gamma}} f(\gamma)
$$

ou

$$
\int_{1} j
$$

6. uma integral em l'st:

i) (Lincaridade) $\int_{\Gamma}(\lambda f+\mu g) \cdots \lambda \int_{\Gamma} f+\mu \int_{\Gamma} y$, para $f, g: \Gamma \rightarrow \mathbb{R}$ continuas $e \lambda, \mu \in \mathbb{R}$.

ii) (Positividade) $S e f(\gamma) \geq 0$ e $\gamma \in \Gamma$ qualquer, culäo $\int_{1} f \geq 0$.

É uma integral de Haar se além de: i) aii) também tem a propriedade.

iii) (Invariancia por transhacia) $\int_{\gamma \in \Gamma^{l}} f(\gamma \delta)=\int_{\gamma \in \Gamma^{\prime}} f(\gamma)$ para todo $\delta \subset \Gamma$ fixado.

Se $\Gamma$ é um grupo de Lio connacto, enta $\Gamma$ ó limitado, logo $\int_{1} 1$ éfinito. Darí, podemos sempre assumir que a integral de Haar satislay $\int_{\gamma<1} 1=1$, o que nos permite considerar a integral de Haar normalizada.

Exemplo 1.1.11 Seja $\Gamma$ um grupo de Lie finito de ordem $|\Gamma|$. Entäo a integral de Haar normalizada em $\Gamma$ é

$$
\int_{\mathrm{l}^{\prime}} f \equiv \frac{1}{|\mathrm{I}|} \sum_{\gamma \subset \mathrm{l}} f(\gamma)
$$

Proposição 1.1.12 Sejam $\Gamma$ um grupo de Lie compacto agindo crn um espaço vetorial n-dimensional $V$, pr a aplicaçio linear cm $\mathrm{GL}(V)$ associada a $\gamma \in \mathrm{I}$. Entäo existe um produlo escalar em $V$ tal que, qualquer que seja $\gamma \in \Gamma$, a mabriz $\rho_{\gamma}$ nume base ortonormal

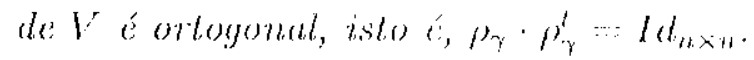

Demonstração $\quad$ Seja $\langle$,$\rangle un prochuto escalar cm V. Definimos$

$$
\langle v, w\rangle_{1}:-\int_{1}\left\langle\rho_{\gamma} v,\left\langle\rho_{\gamma} u\right\rangle\right.
$$

Segue da linearidade e da positividade da integral de Haar que (1.1.12) é um produto interno. Fste produto interno é invariante sob $\Gamma$ pela Invariancia por translaçäo da integral de Haar. Do fatto, paral $v, w \in V$ e $\gamma, \delta \in \Gamma$ temos

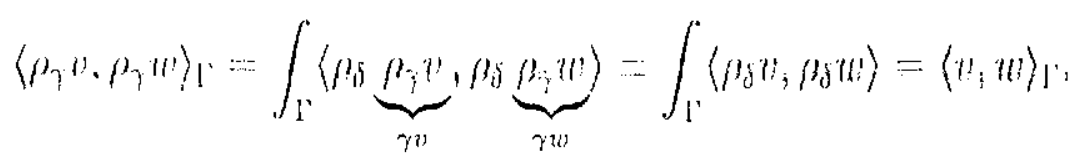


Para $\left\{b_{1}, b_{2}, \cdots, b_{n}\right\}$ base ortonormal de $V$ relativamente ao produto intemo $\langle,\rangle_{1}$, consideramos que $\rho_{\delta}$ é a matriz que representa $\rho(\delta)$ nessa base. Entia, $\rho_{\delta} b_{i}$ é a $i$-ćsina coluna le $\rho_{\delta}$. Alom disso, $\left\langle\rho_{\delta} b_{i}, \rho_{\delta} b_{j}\right\rangle_{\mathrm{r}}=\left\langle b_{i}, b_{j}\right\rangle_{\mathrm{I}}=\delta_{i j}$. Portanto, $\rho_{\delta} \cdot \rho_{\delta}^{t}=I b_{i \times \cdots}$.

Observação 1.1.13 Pela proposiçäo anterior podemos identificar grupos de lie counpactos en $\mathrm{GL}(n)$ com subgrupos fechados de $\mathrm{O}(n)$.

\subsection{Irredutibilidade}

Nesta secão apresentamos un resultado que clecompõe a representação de un grupo de Lie compacto $\Gamma$ cm soma direta de representaçoes mais simples, fue chamanos irredutiveis. Mlén disso, garantimos que esta decomposição sempre existe e apresentimos um exemplo que mostra que en geral esta decomposiçâo não ć única.

\subsubsection{Definições básicas e exemplos}

Seja $\Gamma$ um grupo de Lie compacto agindo linearmente no espaco vetorial $Y$.

Definições 1.2.1 1. Um subespaço $W \subset V$ é chamado $\Gamma$-invariante se $\gamma w \in W$ para qualvuè $\gamma \in \Gamma$ e $w \in W$.

D. Uma representaça ou açăo de $\Gamma \mathrm{em} V$ é dita irredutível se os únicos subespacos I'invariantes de $V$ săo os triviazs, isto $e^{\prime}\left\{0_{V}\right\}$ eV.

3. Um subespaco $W \subset V$ é dito $\Gamma$ - irredutível (ou irredutível se está cluro que o grupo

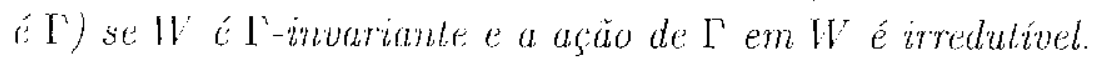

Como exemplo de açoes irredutíveis consideramos as açoes de $\mathrm{SO}(2)$ e de $\mathrm{O}(2)$ sobre o espage vetorial $\mathbb{R}^{2} \equiv \mathbb{C}$ defindas no Exemplo 1.1 .5 , para $K \neq 0$ ).

Una das propriedades fundamentais dis ações dos grupes de Lie compactos é que subespaços invariantes sempre têm complemento invariante.

Proposição 1.2.2 Seja $\Gamma$ um grupo de Lie compacto agindo no espaço vetorial $V$. Seja $W \subset V$ um subespaso $\Gamma$-invariante. Entăo existe um subespaco complementar $\Gamma$-invariante $Z \subset Y$ de $W$ tal que

$$
Y=W \oplus Z
$$

Demonstração Pela Proposição 1.1 .12 existe um produto intomo $\Gamma$-invariante, $\langle,\rangle_{1}$, em $V$. Seja $Z=W^{\prime}-\left\{v \in V ;\langle v, w\rangle_{r}=0, \forall w \in W\right\}$. Como o produto interno á $\Gamma^{\prime}$-invariante entäo $\forall \gamma \in \Gamma, v \in W^{\perp}$ e $w \in W$,

$$
\langle\gamma v, w\rangle_{\Gamma}=\left\langle v, \gamma^{-1} w\right\rangle_{1}=0
$$


ou seja, $\gamma v \in W^{\star}, \forall \gamma \in l^{\prime}$ e $v \in W^{\circ}$. Portanto, $Z-W^{\perp} \subset V$ é um subespaco invariante: e $W$ possui un subespaco complementar invariante.

Segue diretamente destia proposição que toda representagăo de um grupo de Lie pode ser escrita como soma direta de subespaços irredutiveis. Essta decomposição do espaço em que o grupo está agindo é em um certo sentido análoga ì diagonalização de matrizes; que também é feita com o proposito de simplificação dos cálculos.

Corolário 1.2.3 (Teorema da Redutibilidade Completa) Seja $\Gamma$ um grupo de Lie com-

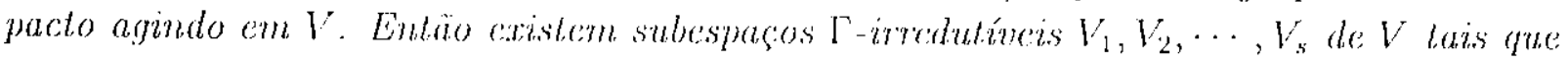

$$
V=V_{1} \oplus V_{2}(\mathrm{D}) \cdot V_{s}
$$

Demonstração Podemos assumir $V$ não nulo. lintũo existe um subespaço $\Gamma$ invariante; considere $V_{1}$ o subespaço não whio de menor dincusão e $\Gamma$-invariante. Pela Proposição 1.1 .12 existe um subespaço $Z \subset V$ l'-invariante e complementar de $V_{1}$. Repetindo o processo em $Z$ obtemos num subesparco $V_{2} \subset V$ r-invariante. Como $V$ ć um espaço vetorial de dinnensão finita, então o processo deve parar permitindo encontrar a decomposiçio (1.2.13).

Os excmplos a seguir ilustram decomposições de espaços vetoriais sob a ação de certos grupos.

Exemplos 1.2.4 1. Consideremos a açăo do $\mathrm{O}(2)$ em $\mathbb{R}^{3}$ como scgue. Seja $\theta \in \mathrm{SO}(2)$ agindo em $\mathbb{R}^{3}$ por rotaçäo de 20 no plano- $(x, y)$ e deixando o cixo-z fixado, isto é, definimos

$$
\theta \cdot(x, y, z)=(x \cos (2 \theta)-y \operatorname{sen}(2 \theta), x \operatorname{sen}(2 \theta)+y \cos (2 \theta), z) .
$$

Considere a reflexăo $\kappa \in \mathrm{O}(2)$ agindo $\mathrm{cm} \mathbb{R}^{3}$ por:

$$
\kappa \cdot(x, y, z)-(x,-y,-z) \text {. }
$$

Podemos decompor o espuco $V$ como $V_{1} \oplus V_{2}$ onde $V_{1}=\{(x, y, 0) ; x, y \in \mathbb{R}\}$ e $V_{2}=$

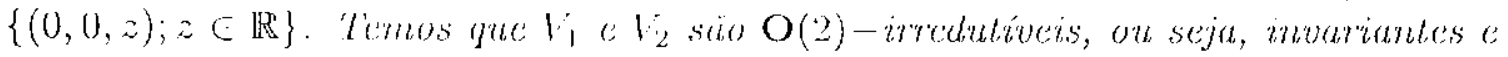
$\mathrm{O}(2)$ age irredulinelmente neles.

2. Existe uma açäo irredulível padräo de $\mathrm{O}(3)$ no espaço vetorial $V$ das matrizes simétricas $3 \times 3$ com traco zero. Tais matrizes bem a forma

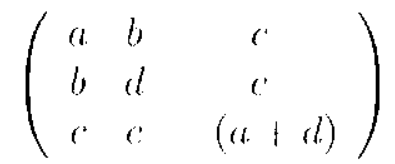

c V tem dimensäo 5. Consideramos a aço do $\mathrm{O}(3) \mathrm{em} V$ por semelhança:

$$
\begin{array}{cl}
\mathrm{O}(3) \times V & \rightarrow V \\
(\gamma, A) & \rightarrow \gamma \cdot A=\gamma^{l} A \gamma
\end{array}
$$


para qualquer $\quad: \in \mathrm{O}(3)$ $A \subset V$. Como $\mathrm{O}(2) \subset \mathrm{O}(3)$, identificamos $\delta \in \mathrm{O}(2)$ com

$$
\left(\begin{array}{ll|l} 
& & 0 \\
& \delta & 0 \\
\hline 0 & 0 & 1
\end{array}\right) \in \mathbf{O}(3)
$$

Tomando $\mathrm{O}(2)$ desta maneira em $\mathrm{O}(3)$ podemos olhar para $\mathrm{O}(2)$ agindo em $\mathrm{V}$. Verificamos entäo que $V_{1}=\left\{\left(\begin{array}{ccc}a & b & 0 \\ b & -a & 0 \\ 0 & 0 & 0\end{array}\right) ; a, b \in \mathbb{R}\right\}, V_{2}=\left\{\left(\begin{array}{lll}0 & 0 & c \\ 0 & 0 & d \\ c & d & 0\end{array}\right) ; c, d \in \mathbb{R}\right\}$, $V_{3}=\left\{\left(\begin{array}{ccc}a & 0 & 0 \\ 0 & a & 0 \\ 0 & 0 & 2 a\end{array}\right) ; a \in \mathbb{R}\right\}$ são $\mathrm{O}(2)$-irredutíveis.

$V=V_{1} \oplus V_{2} \Theta$

Em geral, a decomposição de V em (1.2.13) não é única. Na próxima subsegrio entendemos a origem desta não unicidade e concontranos condiçoes para due uma decomposição do tipo (1.2.13) seja única. () exemplo a seguir mostra esta não unicidade da decomposição.

Exemplo 1.2.5 Seja $V=M_{2}(\mathbb{R})$. Considerumos a açầ de $\mathrm{SO}(2)$ em $V$ pela multiplicaçao de matrizes pela esquerda, isto é, $\theta \in \mathbf{S O}(2)$ e $A \in V$,

$$
\theta \cdot A=R_{\theta} A
$$

Para $V_{1}=\left\{\left(\begin{array}{ll}a & 0 \\ b & 0\end{array}\right) ; a, b \subset \mathbb{R}\right\} \Leftrightarrow V_{2}=\left\{\left(\begin{array}{ll}0 & c \\ 0 & d\end{array}\right) ; c, d \in \mathbb{R}\right\}$, temos que $V=V_{1} \oplus V_{2}$ eque $V_{1}$ e $V_{2}$ são $\mathbf{S O}(2)$-irredutivers. Mas se consideramos $V_{2}=\left\{\left(\begin{array}{ll}8 c & c \\ 8 d & d\end{array}\right) ; c, d \in \mathbb{R}\right\}$, temos $V_{2}^{\prime}$ também $\mathrm{SO}(2)$-irredutínel e $V=V_{1} \oplus V_{2}$.

\subsubsection{Decomposição isotípica}

Apresentamos aqui um resultado que garante a unicidade de una decomposiça do tipo $(1.2 \cdot 13)$.

Teorema 1.2.6 Seja $\Gamma$ um grupo de Lie compacto agindo em $\mathrm{V}$.

a) A menos de $\mathrm{I}$-isomorfismo, existe um número finito de subespacos distintos de $V$ l'-irredulive Dens. Denomos por $U_{1}, L_{2}, \cdots, I_{1}$.

b) Defina $W_{k}$ como sendo a soma de todos os subespacos $W$ C-irredutíneis de $V$ tal que $W$ é $\Gamma$-isomorfo a $U_{k}$. Enläo,

$$
V=W_{1} \in W_{2} \oplus \cdots \oplus W_{1}
$$

Definição 1.2 .7 (Os subespuços $W_{k}$ salo rhamados componentes isotípicas de $V$ do tipo $U_{k}$ para a açào de l'. 


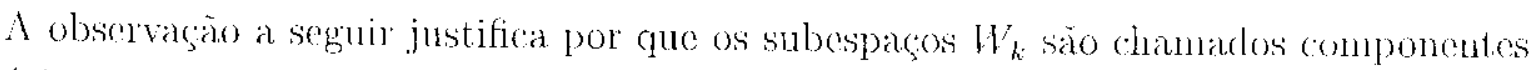
isulípicas e mostra a unicidade da decomposição (1.2.14).

Observação 1.2.8 Por construçăo, a decomposição isotípica (1.2.14) é linica. De fato.

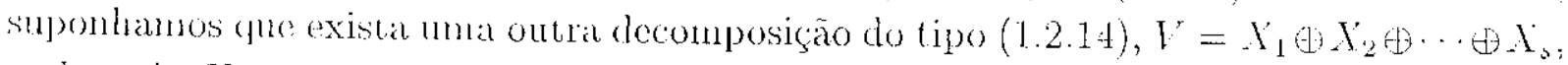

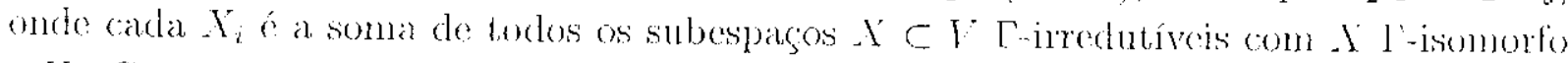
a $U_{i}$. Conno temos somente $U_{i}$ 's entäo $s=t$ e, além disso, pela própria definiçào, segue '1u! $X_{i}=H_{t}$, potanto $X_{1} \oplus X_{2} \oplus \cdots \oplus X_{t}=W_{1} \oplus W_{2} \oplus \cdots \oplus W_{t}$.

Corolário 1.2 .9 a) Se $W^{*} \vee$ é $\Gamma$-irredutivel entäo $\mathrm{W} \subset \mathrm{W}_{k}$ para um único k.

b) Sega $\Gamma$ um grupo de Lite compacto agindo em $V$. Seja $V=V_{1} \oplus V_{2} \oplus \cdots \oplus V_{s}$ uma de:composiço de l' ch soma direta de subespacos $\Gamma$-irredutiveis. Se as representaços

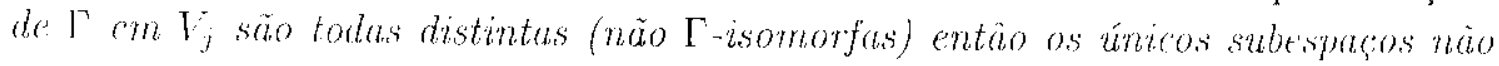
nulos $\Gamma$-irredulávels de $\mathrm{V}$ säo $V_{1}, V_{2}, \cdots, V_{s}$.

\section{Demonstração}

a) Sabonos pelo item a) (lo Teorena 1.2 .6 gue existe somente um munero finito de subespaços l'inredutiveis nào isomorfos que denotanos por $U_{1}, \cdots, U_{t}$. Suponhamos

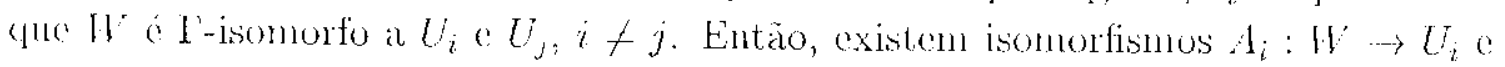
$A_{j}: W \rightarrow U_{j}$ tais que, para $\gamma \in \Gamma, A_{i}(\gamma w)=\gamma A_{i}(w) e A_{j}(\gamma w)=\gamma A_{j}(w)$. Desde que $A_{j}$ a um isonortismo temos que $A_{i} \circ A_{j}^{-1}: U_{i} \rightarrow U_{j}$ e um isomorfismo, o que é unla contradigio. Portanto, $W$ está contido em um único $W_{k}$.

b) Consideranos as componentes isotípicas $W_{k}$ de $r$. Cono cada $V_{j}$ é F-incelutivel, então $V_{j} \subset W_{k}$ para algum $k$. So $V_{i} \subset W_{k} \subset V_{j} \subset W_{k}$, para $i \neq j \subset\{1,2, \cdots, s\}$ entaic, $V_{i}$ e $V_{j}$ sio l'-isomortos a $U_{k}$ : absurdo, pois, temos por hipótesc que as representaçós de I nos $V_{i}$ s sito todas distintas. Logo, $V_{j}=W_{k}$ para algum $k$. Se $I V \neq 06$ un subespaco r-irredutível de $V$, por a) scgue que $W \subset W_{k}$ para algum $K$, mas $V_{j}=H_{k}$ para algum je $V_{j}$ é $\Gamma$-irredutível. Daí, $W=V_{j}$.

Os lemals a seguir loram usados para demonstrar o l'eorema 1.2.6.

Lema 1.2.10 Seja $\urcorner$ um grapo de Lie compacto agindo no espaso velorial W. Suponha gue

$$
W=\sum_{\alpha} U_{c}
$$

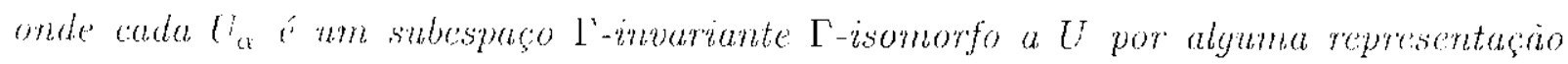

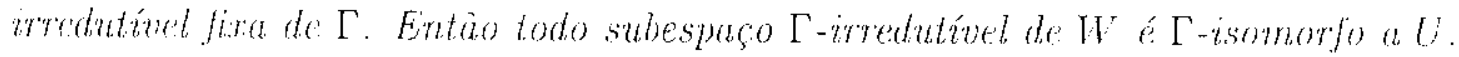

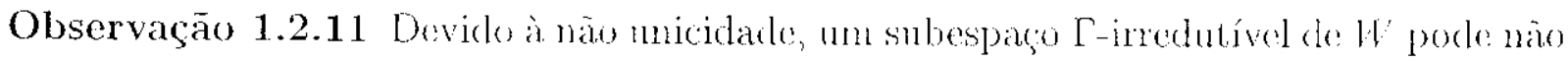
sier un dos $U_{a x}$ 's. O lema diz que, descte que todos os $U_{a}$ 's são l'-isonorfos, todo subespaco le $l$ i l'-isonurto a algum $U_{\alpha}$. 


\section{Demonstração}

(Lemia 1.2.10)

Na mancira cono o lema está enunciado, o conjunto dos indices ex pode ser infinito. Isto näo representia un aumento na generalidade se prineiro mostrarmos que

$$
W=U_{\alpha_{1}} \oplus U_{\alpha_{2}} \oplus \cdots(1) U_{\alpha_{s}},
$$

onde $s$ é um número finito. Para mostrar este fato usamos induçio. Suponhamos que achamos un subesparso

$$
W^{\prime}=U_{r_{1}} \oplus U_{\alpha_{2}} \oplus \cdots U_{r_{1-1}} \subset W
$$

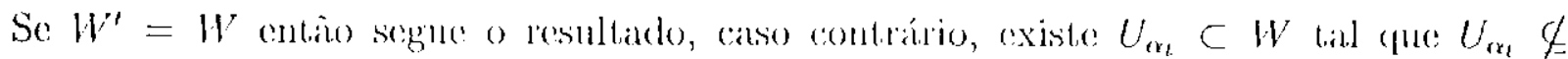
$W^{\prime}$. Além disso, $U_{\alpha_{1}} \cap W^{\prime} \subset U_{\alpha_{t}}$, mas como $U_{\alpha t}$ \& $\Gamma$-irredutível e a interseç̧ão é $\Gamma$-invariante, então $U_{\alpha,} \cap W^{\prime}=\{0\}$. Portantos; a soma $W^{\prime}+U_{\alpha,}$ é a soma direta $W^{\prime \prime}=U_{\alpha_{1}} \oplus U_{\alpha_{2}} \oplus \cdots \oplus U_{\alpha_{t}}$.

Se $W=W^{\prime \prime}$ segue o resultado, caso contrário repetimos o processo. Como $W$ é um espaço vetorial de dinnensino linita então o processiso tem um núnero finito de etapas e (1.2.15) vale para um s suficientemente grande.

Agora mostramos que se $X$ é um subespaço $\Gamma$-irredutível de $W$, então $X$ ó l'-isomorfo a $U$.

Existe $t \leq s$ tal que?

$$
X \nsubseteq U_{\alpha_{1}} \oplus U_{\alpha_{2}} \oplus \cdots \oplus U_{\alpha_{t-1}}
$$

e

$$
X \subset U_{\alpha_{1}} \oplus U_{\alpha_{2}} \oplus \cdots \oplus U_{r_{1, \ldots 1}} \oplus U_{\alpha_{t}}
$$

c, alćm disso, este té ́nico.

Notamos que

$$
X \cap\left(U_{\alpha_{1}} \oplus U_{\alpha_{2}} \oplus \cdots \oplus U_{\alpha_{t-1}}\right)=\{0\}
$$

pois esta intersecgio é un subespaço $\Gamma$-invariante de $X$, e $X$ é, por hipótese, $\Gamma$-irredutível.

Consideramos a aplicaçio projeção $\pi$ dacta por:

$$
\pi: U_{r_{1}} \oplus U_{r_{2}} \oplus \cdots \oplus U_{r_{1}} \rightarrow U_{r_{1}}
$$

Como (1.2.18) está salisfito, para $x=x_{\alpha_{1}}+\cdots+x_{\alpha_{t}} \in X$ temos que $x_{\alpha_{t}} \neq 0$ e pela restrição (isomorfismo) $\pi_{X}=\left.\pi\right|_{X}: X \rightarrow \pi(X)$ temos que

$$
\begin{gathered}
\pi_{X}(\gamma x)=\pi_{X}\left(\gamma\left(x_{\alpha_{1}}+\cdots+x_{\alpha_{t}}\right)\right)= \\
\pi_{X}\left(\gamma x_{\alpha_{1}}+\cdots+\gamma x_{\alpha t}\right)=\gamma x_{\alpha_{t}}=\gamma \pi_{X}\left(x_{\alpha_{1}}+\cdots+x_{\alpha_{t}}\right)=\gamma \pi_{X}(x),
\end{gathered}
$$

ou seja, $\pi_{X}$ é un $\Gamma$-isomorfismo de $X$ em $\pi(X)$. Como $X$ é irredutível cntão $\left.\pi\right|_{X}(X)$ também é irredutivel, nas $\left.\pi\right|_{X}(X) \subseteq U_{\alpha_{t}},\left.\log \pi\right|_{X}(X)=U_{\alpha_{t}}$ e $X$ é C-isonorfo a $U_{\alpha_{t}}$ e então a $U$. 
Lema 1.2.12 Seja $\Gamma$ um trupo de Lir compacto agindo em $\mathrm{V}$. Sejam $\mathrm{X}$ o $Y$ subrspagos de $V$ r-inuariontes lal que näo existem dois subespacos $\Gamma$-irredutíveis $W \subset X$ e $Z \subset Y$

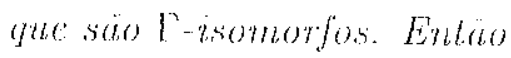

a) $x \cap\}^{\circ}=\{0\}$

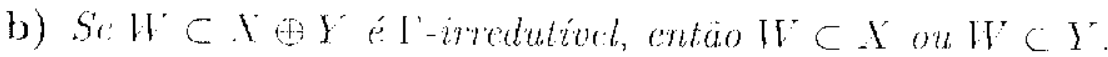

\section{Demonstração}

a) Gomo $X^{*}$ ○ são invariantes, então $X \cap Y$ é invariante. Qualcuer subespaço $A \subseteq X \cap Y^{\prime}$

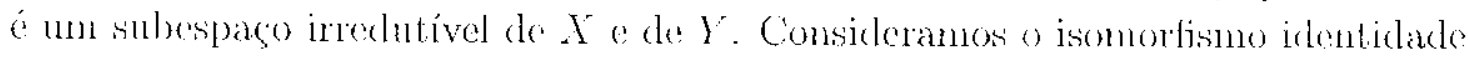

$$
\begin{aligned}
i: A_{X} & \rightarrow A_{Y} \\
a & \mapsto a \\
i(\gamma a)=\gamma a & =\gamma a(a),
\end{aligned}
$$

$\operatorname{logo}, A_{X} \subset \mathrm{X}$ e $A_{Y} \subset Y$ são $\Gamma$-isonorfos, absurdo. Portanto, o único subespaco irredutível em $X \cap Y$ é o nulo. Logo, $X \cap Y=\{0\}$.

b) Seja $W \subset$ I subespaco $\Gamma$-imedutível. Como $X, Y$ e $W$ sào invariantes entio $X \cap W$

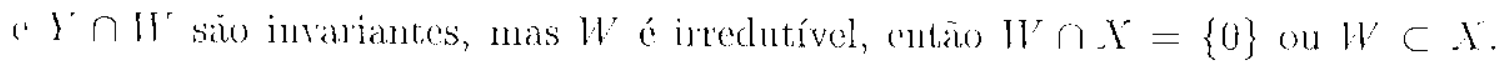
Analogimente, $W \cap Y=\{0\}$ ou $W \subset Y$. Suponhamos que $W \nsubseteq X$ : $W \notin Y$. $W^{\prime} \cap Y^{r}=\{0\}=W \cap Y$. Sejan $\pi_{X}: X \oplus Y^{r} \rightarrow X \in \pi_{Y}: X \oplus Y \rightarrow Y^{\prime}$ projeçoes.

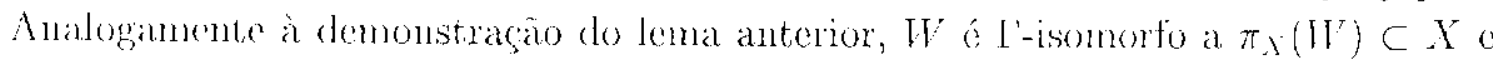

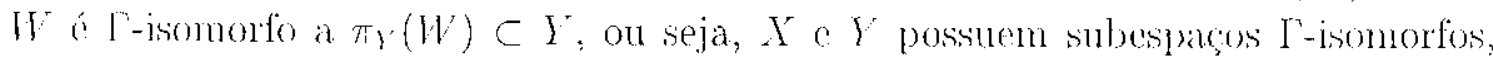
absurclo. Portianto, $W \subset X$ ou $W \subset Y$.

Demonstraçāo (Teorema 1.2.6) Escollamos un subespaço irrechutível $U_{1} \subset V$. Scjic $W_{1}^{\prime \prime}$ a soma de todos os subes]arges invariantes que são ${ }^{\prime}$-isomorfos a $U_{1}$. Se $W_{1}^{\prime} \neq V$, então tomanos seu complementar invaliante $Z$ em $V$ e repitimos o processo em $Z$. Sejam $U_{2} \subset \mathrm{V}$ um subespaco irredutível o $W_{2}^{\prime}$ a soma de todos os subespaços de $Z$ que são $\Gamma$-isomorfus a $U_{2}$. So $W_{1}^{\prime} \oplus W_{2} \neq V$ tomamos un complementar de $W_{2}$ em $Z$, o repitamos o processo. Como a dimensào de $V$ é finita cntão o processo é finito com

$$
V=W_{1}^{\prime} \oplus \cdots \oplus W_{s}^{\prime}
$$

onde carla $W_{h}$ ó a soma de conjuntos $\Gamma$-isomorfos a subespacos irredutiveis do $V$, digamos, isomorfos a $i_{k} \subset V$ e se $k \neq j$, entäo $U_{k}$ não é I'-isomorfo a $U_{j}$.

Verificamos que $W_{k}=W_{k}^{\prime}$. Suponhamos que $U$ é am subespaco incedutivel de $V$. Proto item b) do Lema 1.2.12 U $\subset W_{k}^{\prime}$ para algum índice $h$. Como $W_{k}^{\prime}$ é soma de subespacess l'-isomorles a $U_{k}$ veriticamos a parte a) do teorema.

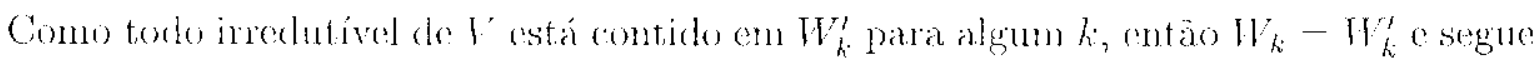
o itemb). 


\subsection{Teoria básica de bifurcação equivariante}

Antes de apresentamos a teoria de bilureaça equivariante, apresentanos na próxina subseção os conceitos básicos de germe e de bifurenģăo.

\subsubsection{Os conccitos de germe c bifurcação}

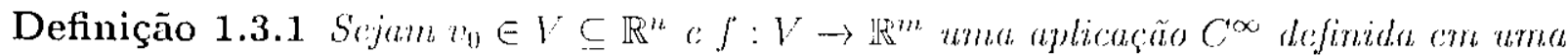
vizinhanģa de $V$. Enkäo g germe de $J$ em vo, ou simplesmentc o germe de $f$, é o conjunto $\left\{g: U \subseteq \mathbb{R}^{n} \rightarrow \mathbb{R}^{m}\right.$ : existe uma vizinhang:a $W$ dr: $v_{0}$ contida cm $U \cap V$ lal que $\left.f\right|_{W}=$ $\left.\left.g\right|_{w}\right\}$ e é denotado por $f:\left(\mathbb{R}^{n}, v_{0}\right) \rightarrow \mathbb{R}^{m}$.

Seja V un espaço vetorial. Consideramos o sistema do tipo

$$
\dot{x}+g(x, \lambda)=0
$$

com $g: V \times \mathbb{R}, 0 \rightarrow V$ um germe de aplicação diferenciável de classe $C^{\infty}$. Os pontos de equilíbrio do sistema são precisamente as soluções de $g(x, \lambda)=0$. Queremos discutir o número de soluções $x$ para diferentes valores de $\lambda$. Como $x(t)=x_{0}$ é solıção de equilíbrio se, e somente se, $\dot{x}=0$, entrio podemos formular o problema com a equação

$$
g(x, \lambda)=0
$$

onde $x$ é chamada variável de estado e $\lambda$ é chanlado parâmetro de bifurcação.

Para cada $\lambda$, seja $n(\lambda)$ o número de $x$ 's para o qual $(x, \lambda)$ é una solução de (1.3.19). Nosso estudo é local; assim, assumimos que (1.3.19) pode somente estar definido em uma vizinhança de uma solução $\left(x_{0}, \lambda_{0}\right)$ onde $n(\lambda)$ varia na vizinhança de $\lambda_{0}$. O conjunto dos

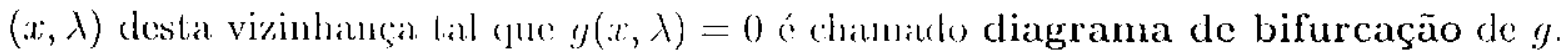

Definiçāo 1.3.2 Assuma que $g\left(x_{0}, \lambda_{0}\right)=0$. Chamamos $\left(x_{0}, \lambda_{0}\right)$ ponto de bifurcação da equação (1.3.19) se, para cada $\lambda$, o número de solucöes $n(\lambda)$ variar conforme $\lambda$ varia numa vizinh $a n c ̧ a$ de $\lambda_{0}$.

Se $\left(x_{0}, \lambda_{0}\right)$ for um ponto de bifurcação, então $(d y)_{x_{0}, \lambda_{0}}=0$, pois pelo Teorema da Função Implícita se $(d g)_{x_{0}, \lambda_{0}} \neq 0$ então (1.3.19) pode ser unicamente resolvida para $x$ como uma função de $\lambda$. Em outras palavras, para carla $\lambda$ perto de $\lambda_{0}$ existe exatamente uma soluçāo de (1.3.19) próxima de $x_{0}$ e, portanto, $n(\lambda)$ não varia. Por conveniência, consideramos germe em uma vizinhança da origem em $V \times \mathbb{R}$. De fato, se o ponto de bifurcagão não for a origen, podemos transladar o sistema de coordenadas c realizar um estudo local en torno da origem. Logo, suponbamos $(d g)_{0,0}=0$.

Agora alpresentimos a teoria de bifureaçio equivariante inciando com funçóes invariantes. 


\subsubsection{Funções invariantes}

Definição 1.3.3 Seja I um grupo de Lie compaclo aghindo num cspaço vclorial V. Dizemos que uma funçâ a valores reais $f: V \rightarrow \mathbb{R}$ é invariante sob a açầ de $\Gamma$, ou é $\Gamma$-invariante, se

$$
f(\gamma x)=\int(x)
$$

para todo $\gamma \in \Gamma$ e $x \in V$.

Pela linearidade da açào de $\Gamma$ é suficiente verificar (1.3.20) para os geradores do grupo $\Gamma$.

Exemplos 1.3.4 a) Seja $\Gamma=\mathbb{Z}_{2} \equiv\{1,-1\}$ agindo não trivialmente sobre $\mathbb{R}$, isto é, $1 \cdot x=x e-1 \cdot x=-x$ para lodo $x \in \mathbb{R}$. Logo, $f: \mathbb{R} \rightarrow \mathbb{R}$ ć $\mathbb{Z}_{2}$-invariante se, $e$ somente se, f é uma funçäo par.

Agora, se $f$ é um polinômio invariante por $\mathbb{Z}_{2}$, chtão todos os seus termos possuem potência par. Portanto, existe un polinômio h: $\mathbb{R} \rightarrow \mathbb{R}$ tal que

$$
f(x)=h\left(x^{2}\right)
$$

b) Consideramos a açao pudrä̀ do grupo $\mathrm{S}^{1}$ sobre $\mathrm{V}=\mathbb{R}^{2} \equiv \mathbb{C}: 0 \cdot z=e^{i 0} z$. (ver Excmplo 1.1.5)

Uma funçio $f: \mathbb{C} \rightarrow \mathbb{R}$ ć $\mathrm{S}^{1}$-invarianté quando

$$
f(z)=f\left(c^{i \theta} z\right)
$$

para todo $z \in \mathbb{C}_{e} \theta \in \mathrm{S}^{1}$. A aplicaçiò que a cada $\theta \in \mathrm{S}^{1}$ associa $e^{i \theta} z$ forma um círculo centrado na origen e de raio $|z|$, cntäo as funçöes que são $\mathrm{S}^{1}$-invariantes säo aquelas constanles nesto círculo.

Mostramos que se $\int$ é um polinômio $\mathrm{S}^{1}$-invarianle em $\mathbb{C}$ então existe um polinômio $h: V \rightarrow \mathbb{R}$ tal que

$$
f(z)=h(z \bar{z})
$$

Escrevamos $f$ nas coordenadas $z, \bar{z} \in \mathbb{C}$,

$$
f(z)=\sum a_{\alpha \beta \beta} z^{\mathrm{cr}} \bar{z}^{\beta}
$$

onde $a_{\boldsymbol{\alpha} \beta} \in \mathbb{C}$. Exigimos que os coeficientes sejam complexos de tal maneira que o polinomio f seja real. Desde que f éral, $f=\bar{J}$ e contäo

$$
\overline{a_{i j \mathrm{a}}}-a_{\left(\mathrm{r}_{i}\right)}
$$

Por (1.3.23) temos que

$$
f\left(r^{i \theta} z\right)=\sum a_{c k / \beta} e^{i \theta(c-\beta)} z^{\alpha x} z^{i \beta}
$$


Por (1.9.80) lemos que

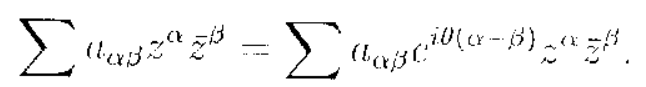

Logo,

$$
a_{\alpha \beta \beta}=a_{\alpha, \beta} e^{i \theta(\alpha-\beta)} \Leftrightarrow \alpha=\beta \text { ou } a_{n ; \beta}=0 .
$$

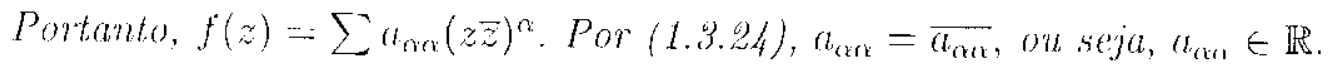

Se consideramos o polinômio $h: \mathbb{R} \rightarrow \mathbb{R}$, tal que, $h(x)=\sum a_{\text {or }}(x)^{\alpha}$, chtäo $f(z)=$ h. (zت) como afimalo em (1.3.20).

c) Seja l'= $\mathrm{D}_{n}$ com sur açäo padrâo sobre $V=\mathbb{C}$, ou seja, a acuo do Exemplo 1.1.5-4 tomando $\Lambda=1$ e $\theta=\frac{2 h \pi}{n}, h=0, \ldots, n-1$. Verificamos que para tolu polanomio

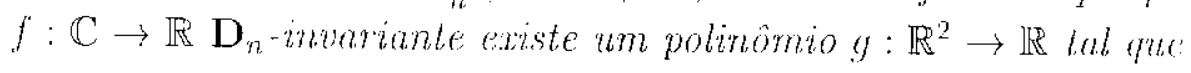

$$
f(z)=g\left(z \bar{z}, z^{n}+\bar{z}^{n}\right) \text {. }
$$

Para isto serumos a idéia do item anterion. Escrevenos f na forma (1.3.93). Desde

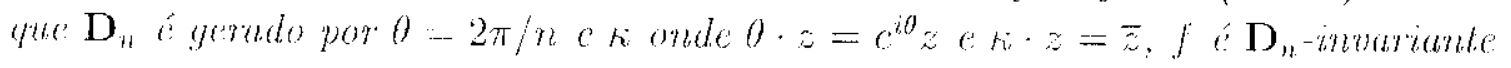
se satisfaz

1. $f(\theta z)=f(z) \Leftrightarrow(1.3 .26)$ vale para $\theta=2 \pi / n$

2. $f(r, z)=f(z) \Leftrightarrow a_{r \beta}=a_{\beta \alpha}$.

Lm suma, fé $\mathbf{D}_{n}$-invariante se, e somente se,

a) $a_{(a, j)} \subset \mathbb{R}$

b) $a_{a k j}-a_{k \beta k}$

c) $a_{n ;}=0$ ou $\alpha \equiv \beta(\bmod n)$, isto é, ou $\alpha-\beta=h n ; k \in \mathbb{Z}$

Rivesuremos (1.3.93)da seguinte forma:

$$
f(z)=\sum A_{\alpha \beta}\left(z^{\alpha-\beta}+z^{\beta} \bar{z}^{\alpha}\right)
$$

onde

$$
A_{\alpha \beta \beta}=\left\{\begin{array}{l}
a_{n \beta} \text { se } \alpha \neq \beta \\
a_{\alpha_{2} \beta} \text { se } \alpha=\beta
\end{array}\right.
$$

Faloramos con (1.3.98) alguns termos e usamos c) para chegar om

$$
f(z)=\sum_{j, k} B_{j k}(z z)^{j}\left(z^{k n}+\bar{z}^{k n u}\right)
$$

para certos $B_{j k}$. Finulmente, usando a igualdade

$$
\left(z^{k n}+\bar{z}^{k n}\right)=\left(z^{n}+\bar{s}^{n}\left(z^{(k-1) n}+\bar{z}^{(k-1) n}\right)\right) \quad(z z)^{n}\left(z^{(k-2) n}+\bar{z}^{(k-2) n}\right)
$$


indutimumente em $k$ rescenle, obtemos

$$
f(z)=\sum_{l, m} C_{l m}(z \bar{z})^{l}\left(z^{n}+\bar{z}^{n}\right)^{m}
$$

para certos cotficientes $C_{\text {in }}$. Se consideramos $g: \mathbb{R}^{2} \rightarrow \mathbb{R}$ tal que

$$
g(x, y)=\sum_{l, m} C_{m}(x)^{l}(y)^{m}
$$

mostramos a afirmacio (1.3.207).

Dado nin grupo de Lie compacto l' é possivel encontrar um subconjunto finito de polinomios l'-invarianues $\mu_{1}, \cdots, \mu_{\mathrm{s}}$ tal que todos os polinomios invariantes polem ser escritos como fungrio polinomial de $\mu_{1}, \cdots, \mu_{s}$. Este conjunto, que pode não ser único, gera o conjunto dos polinomios I'-invariantess e é chamado base de Hilbert. () conjunto dos

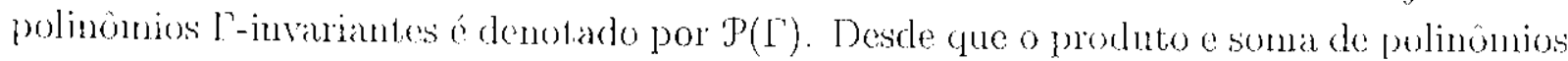
T-invariantes sion ainda l'-invariantes, então $\mathcal{P}(\Gamma)$ ó un anel. Un resultado importante, iniciado por Hibert e plovado por Weyl [1946], o o teorema que segule. Ressaltamos que a chemonstraga é técnica e não foi estudada em detalhes.

Teorema 1.3 .5 (Hilbert o Weyl): Seja $\mathrm{\Gamma}$ um grupo de Lite comparto agindo em V. Entia thiste uma base de IJibert pora o anel $\mathrm{P}(\Gamma)$.

Demonstração [10], cap. XII

Observação 1.3.6 Pela Proposifgão 1.1.12 podemos assunir um grupo de Lie compacto $\Gamma$ como sulogrupo do $\mathrm{O}(n)$. Assim, a norma

$$
\|x\|^{2}=x_{1}^{2}+x_{2}^{2}+\cdots+x_{n}^{2}
$$

Gsempre T-invariante.

Un resultado anćlogo au Teorema 1.3.j tanbém vale para as funçôes analíticas reais, já que estas podem ser escritas como séries de Taylor. Além disso, o Teorema do Schwarz[1975], fue aprosontanos abaixo, é un resultado deste tipo que vale para germes $C^{\infty}$ (Deliniça 1.3.1) quilndo o grupo de Lie é compacto.

Definiçāo $1.3 .7 \varepsilon_{s}$ denota o ancl dos germes $C^{\infty}$ de $\mathbb{R}^{s}$ em $\mathbb{R}$ e E (T) denola o anel dos germes $C^{+\infty}$ T-inuminates de $\mathrm{V}$ em $\mathrm{R}$.

Teorema 1.3.8 (Schuurz [1975]): Seja $\Gamma$ um grupo de Lic compacto agindo cm V. Seja $\mu_{1}, \cdots, h_{s}$ wha base de Hibert para $\mathcal{P}(\Gamma)$. Seja $f \subset \mathcal{E}(\Gamma)$. Entio existe um grome suave $h \in E(s)$ lal que

$$
f(x)-h\left(\mu_{1}(x), \cdots, \mu_{s}(x)\right)
$$

Demonstração $\quad[10]$, cap. XII $\xi 6(b)$. 
Definição 1.3.9 O conjunlo dos polinômios L'-invariantes lem uma relação se existe um polinônio mïu-nulo $r\left(y_{1}, \cdots, y_{s}\right)$ lab rus.

$$
r\left(\mu_{1}(x), \cdots, \mu_{s}(x)\right)=0 .
$$

O ancl $\mathcal{P}(\Gamma)$ é un ancl polinomial se cle tem una base de Hilbert sem relação.

Um excmplo de un grupo agindo en um espaço vetorial tal que $\mathcal{P}(\Gamma)$ não é um anel polinomial é dado por $\Gamma=\mathbb{Z}_{2}$ agrinto on $\mathbb{R}^{2}$, onde a agró do elenento $-1 \in \mathbb{Z}_{2}$ é definida por $x \mapsto-x$. Então $\mathfrak{T}\left(\mathbb{Z}_{2}\right)$ é o anel gerado pelos numónios de grau par, isto é, pela base de Hilbert

$$
\mu_{1}=x_{1}^{2}, \mu_{2}=x_{1} x_{2}, \mu_{3} \cdots x_{2}^{2}
$$

mas esta base tem a relingia

$$
\mu_{1} \mu_{3}-\mu_{2}^{2} \equiv 0
$$

Na verdade, é possível mostrar neste exemplo que nenhuma base de Hlibert elimina todas as relaçōes, portanto $\mathcal{P}\left(\mathbb{Z}_{2}\right)$ nä̀o é unn anel polinominial.

O resultado a seguir permite-nos determinar se man darda base de Hilbert $\mu_{1}, \cdots, \mu_{s}$ torna $\mathcal{P}\left(\mathbb{Z}_{2}\right)$ um ancl polinomial. Definamos a aplicação $\rho: V \rightarrow \mathbb{R}^{s}$, chamada discriminante de $\Gamma$, por:

$$
\rho(x)=\left(\mu_{1}(x), \cdots, \mu_{s}(x)\right) .
$$

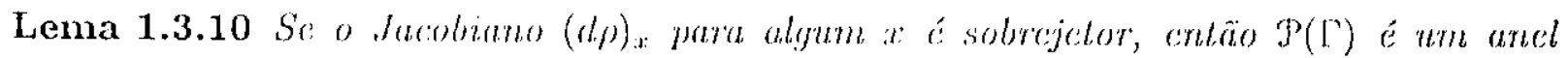
polinomial.

Aplicando o lena acima 110 exemplo onde $\Gamma=\mathbb{Z}_{2}$, temos $\rho\left(x_{1}, x_{2}\right)=\left(x_{1}^{2}, x_{1} x_{2}, x_{2}^{2}\right)$ e $(d \rho)_{x}: \mathbb{R}^{2} \rightarrow \mathbb{R}^{3}$. Assim, ć impossivel $(d \rho)_{x}$ ser sobrejetor para algum $x$. Já no Excmplo 1.3.4c $\mathcal{P}\left(\mathbf{D}_{n}\right)$ é um anel polinomial. De fato, de (1.3.27) temos que

$$
\mu_{1}=z \bar{z}, \mu_{2}=z^{n}+\bar{z}^{n}
$$

é uma base de Hilbert do $\mathcal{P}\left(\mathbf{D}_{n}\right)$ o

$$
(d p)_{z}-\left(\begin{array}{cc}
\bar{z} & z \\
n z^{n-1} & n \bar{z}^{n-1}
\end{array}\right)
$$

Temos que det $(d \rho)=n\left(\bar{z}^{n}-z^{n}\right)$ é năo nulo para $z \neq 0$ a, entiào, $(d \rho)_{z}$ é sobrejetor para algum $z$ não nulo e, pelo Lema 1.3.10, $\mathcal{P}\left(\mathbf{D}_{n}\right)$ é um ancl polinomial.

Até mesmo quando $\mathfrak{S}(\Gamma)$ é um anei polinomial a unicidade de (1.3.29) para germes não analíticos não precisa ser satisfeita. Por cxemplo, $\mathbb{Z}_{2}$ agindo em $\mathbb{R}$ com a ação padrão, $\left\{x^{2}\right\}$ é uma base de Hilbert de $\mathfrak{P}\left(\mathbb{Z}_{2}\right)$ e pelo Teorena 1.3 .8 todo germe $f \in \mathcal{E}\left(\mathbb{Z}_{2}\right)$ pode ser escrito como $f(x)-h\left(x^{2}\right)$, onde he é mn germe suave de $\mathbb{R}$ cm $\mathbb{R}$. Definamos o germe suave

Notemos que

$$
h(x)=\left\{\begin{array}{cc}
e^{-1 / x} & \text { se } x<0 \\
0 & \text { sit } x \geq 0
\end{array}\right.
$$

$$
f(x)=h\left(x^{2}\right)+k\left(x^{2}\right),
$$

pois $k\left(x^{2}\right)=0$ para todo $x \in \mathbb{R}$. Logo, a unicidade fallan para este cxomplo. 


\subsubsection{Aplicações lineares equivariantes}

Consideranos $\Gamma$ mun grupo de Lie compacto agindo linearmente no espacso vetorial $V$.

Definição 1.3.11 Uma aplicaçào $F: V \rightarrow V$ conula com $\Gamma$, ou é $\Gamma$-equivariante, se

$$
F(\gamma v)=\gamma F(v)
$$

para todo $\gamma \in I^{\prime}: v \in V$.

Exemplos 1.3.12 1. Consideramos a aৎ̧a padrüo de $\mathrm{SO}(2)$ em $\mathbb{R}^{2}$ definida pela rotaçao de $\theta \in \mathrm{SO}(2)$. Isto s,

$$
R_{0}-\left(\begin{array}{cc}
\cos \theta & -\operatorname{sen} \theta \\
\operatorname{sen} \theta & \cos \theta
\end{array}\right)
$$

agindo em

$$
\mathbb{R}^{2} \equiv\left\{\left(\begin{array}{l}
x \\
y
\end{array}\right) ; i, y \in \mathbb{R}\right\}
$$

pela multiplicaçăo de malrizes. Verificamos facilmente rue $A=\left(\begin{array}{ll}a & b \\ c & d\end{array}\right) \in M_{2 \times 2}(\mathbb{R})$ comuta com $\mathrm{SO}(2)$ se, e somente se, $a=d$ e $b=-c$. Portanto,

$$
A=\left(\begin{array}{cc}
a & -c \\
c & a
\end{array}\right)=\left(a^{2}+c^{2}\right)\left(\begin{array}{cc}
\cos \varphi & -\operatorname{sen} \varphi \\
\operatorname{sen} \varphi & \cos \varphi
\end{array}\right)
$$

onde $\varphi$ é tal que $\cos \varphi=\frac{a}{a^{2}+c^{2}} e \operatorname{sen} \varphi=\frac{r}{a^{2}+c^{2}}$. Conctuímos que uma aplicaşão linear de $\mathbb{R}^{2}$ em $\mathbb{R}^{2}$ comuta com $\mathrm{SO}(2)$ se, e somente se, é da forma (1.3.31).

2. Consideramos a açăo padrão de $\mathrm{O}(2)$ em $\mathbb{R}^{2}$. Suponhamos que $M \in M_{2 \times 2}(\mathbb{R})$ comuta com $\mathrm{O}(2)$. Desde que $\mathrm{SO}(2) \subset \mathrm{O}(2)$ então $M$ deve satisfazer (1.3.31), isto é, $M=\left(\begin{array}{cc}m & -n \\ n & m\end{array}\right)$, parm $m, n \in \mathbb{R}$. Alem disso, $M$ deve comular com $\kappa=\left(\begin{array}{cc}1 & 0 \\ 0 & -1\end{array}\right)$ e entâo, $n=0$. Portanto,

$$
M=\left(\begin{array}{cc}
m & 0 \\
0 & m
\end{array}\right)-m\left(\begin{array}{ll}
1 & 0 \\
0 & 1
\end{array}\right)
$$

é un múlliplo da idenlidade.

Definição 1.3.13 Uma representação de um grupo T sobre um espaço vetorial $V$ é absolutamente irredutivel se as únicas aplicacoes lineares que comulam com $\Gamma$ são as múltiplas da identidade.

Para justificar esta terminologia temos:

Lema 1.3.14 Seja l'um. grapo de Lie compacto agindo em V. Se a açăo de $\Gamma$ é absolutamente irredutivel, entäu ela é irredutível. 
Demonstração Suponhanos que $\Gamma$ age não irredutivelmente sobre $V$. Então existe $1 \mathrm{~m}$ subespaço próprio $W \nsubseteq \mathrm{V}$ I'-invariante e, pela Proposicão 1.2 .2 , sen complemento $W^{L}$ en $V$ tambén ó l’-invariante. Delinames $\pi: W \oplus W^{\perp} \rightarrow V$ a projeça sobre $W$. Para qualquer $v=v_{1}+v_{2} \in V=W \in W^{\perp}$, onde $v_{1} \in W$ o $v_{2} \in W^{\perp}$,

$$
\pi(\gamma v)=\pi\left(\gamma v_{1}+\gamma v_{2}\right)=\gamma v_{1}=\gamma \pi(v)
$$

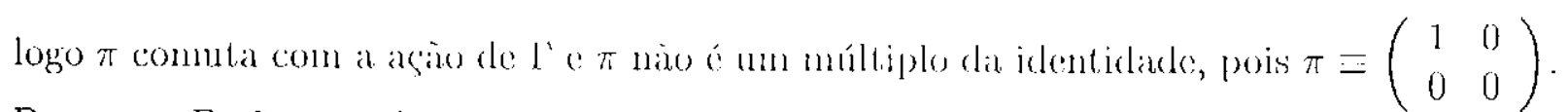
Portanto, $\Gamma$ não age absolulamente irredutivehnente solsre $V$.

Lema 1.3.15 Sejam $\Gamma$ um grupo de Lie compacto ayindo em $V, A: V \rightarrow V$ uma aplicaçăo lincar que comula com $\Gamma \mathrm{eW} \subset V$ subespaço irredulível. Enbäo:

i) $A(W)$ é invarianle

ii) $A(W)=\{0\}$ ou as representaçós de $\Gamma$ cm W e $A(W)$ säo $\mathrm{I}^{2}$-isomorfas

\section{Demonstração}

i) Scja $z=A(w) \in A\left(W^{\prime}\right)$ para algum $w \in W$, então

$$
\gamma z=\gamma A(w) \cdots A(\gamma w) \in A(W)
$$

e $\gamma z \in A(W)$, ou sejal, $A(W)$ é invariante.

ii) Para qualquer $v \in k e r A r \gamma \subset \mathrm{I}$

$$
A(\gamma v)=\gamma A(v)=\gamma()=0
$$

Logo, ker $A$ é invariante o que implica (ker $A) \cap W$ invariante. Como $W$ ó irredutível, entào $W \subset \operatorname{ker} A$ on $W \cap(\operatorname{ker} A)=\{0\}$. Se $W \subset(\operatorname{ker} A)$, então $A(W)=\{0\}$ ou se $W \cap(\operatorname{ker} A)=\{0\}$, chtão $W$ e $A(W)$ sũo $\Gamma$-isomorfos.

Este lema implica que:

Teorema 1.3.16 Seja l'um grupo de Lie compacho argindo no espaso vetorial $V$. Decomponta $V$ cun componentes isotipicas

$$
V=W_{1} \oplus W_{2} \oplus \cdots\left(\Phi W_{s}\right.
$$

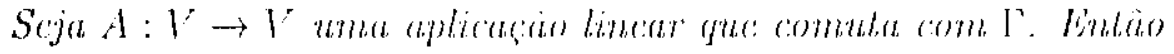

$$
A\left(W_{k}\right) \subset W_{k}
$$

para cada $k=1,2, \cdots, s$. 
Demonstração Pela descrição de caula $W_{k}$ temos que

$$
W_{k}=V_{1} \oplus \cdots(1) V_{r}
$$

é $\Gamma$-isomorfo a um subespaco $U_{k}$ irredutivel de $V$. Cada $V_{j}$ cirredutivel e pelo Lema anterior $A\left(V_{j}\right)$ é invarianté e $A\left(V_{j}\right)=\{0\}$ ou $A\left(V_{j}\right)$ é $\Gamma$-isomorfo a $U_{k}$. Se $A\left(V_{j}\right)=\{0\}$ então $A\left(V_{j}\right) \subset W_{k}$, pois $W_{k}$ é subespaço. Se $A\left(V_{j}\right)$ é $\Gamma$-isomorfo a $U_{k}$ então $A\left(V_{j}\right) \subset W_{k}$, pois $W_{k}$ ća soma de todos invariantes I'-isomortos a $U_{k}$. l'ortanto, $A\left(V_{j}\right) \subset W_{k}$ para cada $j$. Pela linearidade de $A$,

$$
\begin{gathered}
A\left(V_{1}\right) \oplus A\left(V_{2}\right) \oplus \cdots \oplus A\left(V_{r}\right) \subset W_{k} \\
A\left(V_{1} \oplus \cdots \oplus V_{r}\right) \subset W_{k} \\
A\left(W_{k}\right) \subset W_{k} .
\end{gathered}
$$

Este tcorema garante que un aplicação lincar Г-equivariante deixa toda componcnte isotípica invariante.

\subsubsection{Aplicações não lineares equivariantes}

O objetivo agora á apresentam man mancira eficiente de descrever aplicaçoes não-lineares que comutan com a ação de un grupo.

Consideramos $\Gamma$ um grupo de Lie compacto agindo num espaço vetorial $V$.

Lema 1.3.17 Sejam $f: V \rightarrow \mathbb{R}$ uma funs:äo $\Gamma$-invariantc c $g: V \rightarrow V$ uma aplicação $\Gamma$-equivarianle. Enläo, $f y: V \rightarrow V$ é I'-cquivartanle.

Demonstração $\quad(f g)(\gamma v)=f(\gamma v) g(\gamma v)=f(v) g\left(\gamma^{v} v\right)=\gamma f(v) g(v)=\gamma(f g)(v)$ $\Rightarrow(f g)(\gamma v)=\gamma(f g)(v)$

Se consideramos a açăo padrão de $\mathbb{Z}_{2}$ em $\mathbb{R}$, conclúnos que as aplicaçóes $g: \mathbb{R} \rightarrow \mathbb{R}$ $\mathbb{Z}_{2}$-equivariantes sius as applicaçoes inponres.

Sejam $\overrightarrow{\mathcal{P}}(\Gamma)$ o espaço das aplicaçöes polinomiais $\Gamma$-equivariantes de $V$ em $V$ e $\overrightarrow{\mathcal{E}}(\Gamma)$ o espaço dos germes na origem $\Gamma$-ecpuivariantes $C^{(x)}$ de $V$ en $V$. O Lema 1.3.17 implica que $\vec{\beta}(\Gamma)$ á um módulo sobre o ancl dos polinômios $\Gamma$-invariantes $\mathcal{P}(\Gamma)$. Analogamente, $\overrightarrow{\mathcal{E}}(\Gamma)$ é um módillo sobre o anel $\mathcal{E}(\Gamma)$.

Os resultados para $\Gamma=\mathbb{Z}_{2}$ em símbolos sĩo:
a) $\overrightarrow{\mathcal{P}}\left(\mathbb{Z}_{2}\right)=\mathfrak{P}\left(\mathbb{Z}_{2}\right)\{x\}$
b) $\vec{\varepsilon}\left(\mathbb{Z}_{2}\right)=\varepsilon\left(\mathbb{Z}_{2}\right)\{x\}$. 
Dizemos que as aplicaçoes polinomiais l'-equivariantes $g_{1}, \cdots, y_{r}$ geman a módulo $\vec{\jmath}(\Gamma)$ sobre o ane $\mathcal{P}(\Gamma)$ se todo $\Gamma$-cepuivariante g pode ser escrito como

$$
g=f_{1} g_{1}+f_{2} g_{2}+\cdots+f_{r} g r
$$

para polinomios $\Gamma$-invarirantes $f_{1}, f_{2}, \cdots, f_{r}$. Uma definição similar porle ser foita para $\vec{E}\left(l^{\prime}\right)$. O próxino teorema é correspondente do Teorema 1.3.5 para o caso cequivariante.

Teorema 1.3.18 Seja T um grupo de Lie compacto agindo em V. Entüo existe um. mumero finilo de aplicaşoes polinomiais $g_{1}, \ldots, g_{r}$ I'equinuriantes que geram o módulo $\vec{\jmath}(\Gamma)$

Demonstraçāo [10], (ap. XII, §̧6(c).

Agora vanus ver uma versào do Teorema de Schwarz para as aplicaçoos $\Gamma$-equivariantes. Esta foi provada por Poénarn em 1976.

Teorema 1.3.19 (Pónaru[1976]) Sejam $\Gamma$ um grupo de Lie compacto o g, $, \cdots, g_{r}$ ge-

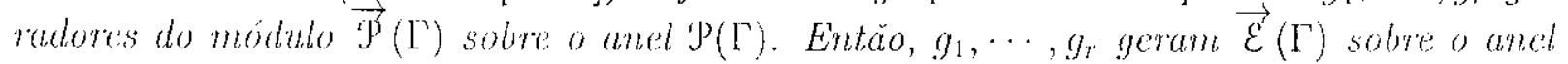
$\varepsilon(\Gamma)$

Demonstração $\quad[10]$, «ap) XII, $\S 6(c)$.

(Os exmplos a seguir são obtidos diretamente a partir dos Exemplos 1.3 .4 o do Teorema 1.3.19.

Exemplos 1.3.20 a) Seja $\Gamma=\mathbf{S}^{1}$ com a açâa padräo sobre $\mathbb{C}$, isto é, $0 \in \mathrm{S}^{1}$ ez $z \mathbb{C}$,

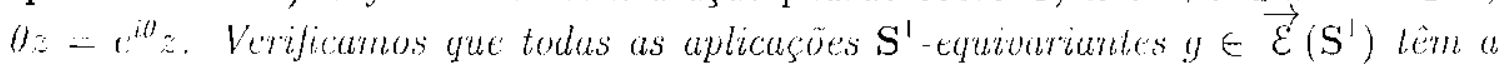
formu.

$$
g(z)=p(z \bar{z}) z+q(z \bar{z}) i z
$$

onde p e q sào germes de funcöes em $\mathbb{R}, 0$.

b) Considerumos $\Gamma^{2}=\mathrm{O}(2)$ agindo com a açăo padräo sobme $\mathbb{C}$. Afimamos que toda a uplicascio $\mathrm{O}(2)$-truivuriante $g \in \overrightarrow{\mathcal{E}}(\mathrm{O}(2))$ tem a forma

$$
g(z)=p(z \bar{z}) z
$$

c) Seja $\Gamma=\mathbf{D}_{n}$ com a aça padräo solre $\mathbb{C}$. Todo o game $\mathbf{D}_{n}$ equivariante temn a forma

$$
y(z)-p(u, v) z+q(u, v) \div \cdots \cdot
$$

onde $u=z \bar{s} u n=z^{n}+\bar{z}^{n}$.

Para finalizar osta segão discutimos quando um germe g l'epuivariante á eserito de forma ínical comos en (1.3.3.34). 
Definição 1.3.21 Dizemos que $g_{1}, \cdots, g_{r}$ geram $\vec{P}(\Gamma)$ livrcmente sobre $\mathcal{P}(\Gamma)$, ou que $\overrightarrow{\mathfrak{P}}(\mathrm{l})$ é um módulo livre sobre $\mathfrak{\jmath}(\mathrm{I})$, se a relaçăo

$$
f_{1} g_{1}+\cdots+f_{r} g_{r} \equiv 0
$$

onde $f_{j} \in P(\Gamma)$, implicar que.

$$
f_{1} \equiv \cdots \doteq f_{r} \equiv 0
$$

Conn csta estrutura temos que se $g_{1}, \cdots, g_{r}$ geram $\overrightarrow{\mathcal{P}}(\Gamma)$ livremente sobre $\cdot \mathcal{P}(\Gamma)$ então

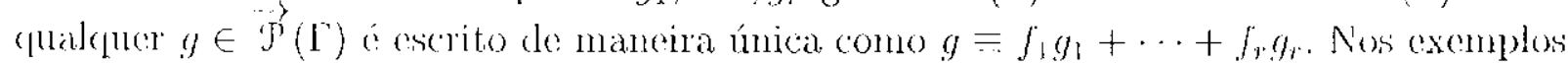
anteriores os módulos são livres.

\subsection{Quebra de simetria em bifurcação de pontos de equilíbrio}

Aqui, estudamos bifurcargão de pontos de equilíbrio para sistemas de EDO

$$
\frac{d x}{d t}+g(x, \lambda)=0
$$

Suponos que $g: \mathbb{R}^{n} \times \mathbb{R} \rightarrow \mathbb{R}^{n}$ satisfaz

$$
g(\gamma x, \lambda)=\gamma g(x, \lambda)
$$

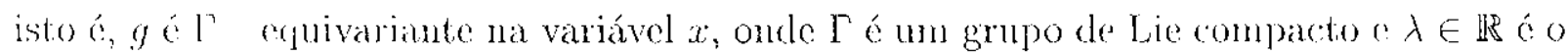
paràmetro de bifurcação. Uma solução de equilíbrio $(x, \lambda) \in \mathbb{R}^{n} \times \mathbb{R}$ satisfaz $d x / d t=0$, isto $e_{\text {, }}$

$$
g(x, \lambda)=0
$$

Focalizamos agui em simetrias que uma soluçà $x$ pode possuir.

\subsection{1 Órbitas e subgrupos de isotropia}

Seja I un grupo de Lie agindo no espaço vetorial $V$.

Definições 1.4.1 A órbita de $x \in V$ pela açâa de $\Gamma$ é o conjunto

$$
\Gamma x=\{\gamma x ; \gamma \in \Gamma\} \text {. }
$$

O subgrupo de isotropia de $x \in V$ é

$$
\Sigma_{x}=\{\gamma \in \Gamma ; \gamma=x\}
$$

Suponkanos $f: V \rightarrow V \Gamma$-equivariante, então $f(\gamma x)=\gamma f(x)$ para todo $\gamma \in \Gamma$ e $x \in V$. Se $x \in V$ ó lal que $f(x)=0$ então $f(\gamma x)=\gamma f(x)=\gamma()=0$, isto é, se $x$ ć una solução, crutia tonlos os elementos da sua órbita $\Gamma x$ também säo.

O lema a seguir mostra qual a relayão entre os subgrupos de isotropia de clementos na mesma ólitita. 
Lema 1.4.2 Pontos na mesma orbita pela açüo de $\Gamma$ possuem subyrapos de isolropia conjugados, ou seja,

$$
\Sigma_{\gamma: x}=\gamma \Sigma_{r} \gamma^{\prime}
$$

Demonstração Sojann $x \in V$ e $\gamma \in L^{2}$. Para $o \in \Sigma_{r}$, queremos mostrar que $\gamma \sigma \gamma^{-1} \in \Sigma_{\gamma x}$. De fato, $\gamma \sigma \gamma^{-1}(\gamma x)=\gamma o x=\gamma x \in \sum_{\gamma x x}$. Portanto, $\Sigma_{\gamma x}$ ? $\gamma \Sigma_{x} \gamma^{\cdots 1}$.

Por outro lado, se $\delta \in V_{\gamma,}$, entào $\delta \gamma x=\gamma x$ e $\gamma^{-1} \delta \gamma x=\gamma^{-1} \gamma x=x$, su seja, $\gamma^{-1} \Sigma_{\gamma x} \gamma \subseteq \Sigma_{x}$ Loggo, $\Sigma_{\gamma: x} \subseteq \gamma \Sigma_{x} \gamma^{\cdots 1}$.

Un método convenionte de descrever geometricanente a ação de nun grupo de Lie I' an $V$ a agrupar en un conjunto $W$ todos os pontos de $V$ que tém subgrupos de isotropia conjugaros. Dizemos que Wé um tipo de órbita da açan.

Ilustranos estas idéias considerando a ação do grupo dieclaal $\mathbf{D}_{n}$ en $\mathbb{C}$ gerau por

$$
\begin{gathered}
r: \varkappa \mapsto \bar{z} \mathrm{c} \\
\xi: z \mapsto e^{2 \pi i / n} z .
\end{gathered}
$$

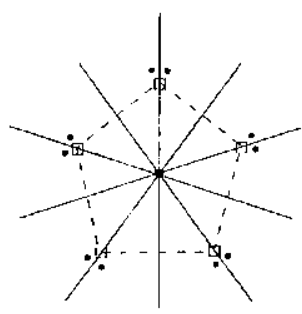

Figura 1.1: Polígono regular de 5 lados

Geonetricannente, identificamos a ação do grupo $\mathrm{D}_{n}$ como sendo as simetrias do polígono regular de n lados 110 plano centrado na origen. Na Figura 1.1 tenos o polígono de 5 lados. Deduzimos os tipos de órbitas da ação do grupo $\mathrm{D}_{n}$, onde os resultados dependen se $u$ ć par ou ímpar, no nosso caso $n$ é ímpar. Se na Figura 1.1 consideranos o vértice com símbolo $\square$. Notamos que a ação dos clementos $\xi^{j}$ e r, para $j=0,1,2,3,4$, leva este em cada um dos ontros vértices, ou seja, os vértices constituem una órbita. O subgrupo de isotropia de $u 1 n$ vértice no eixo real é o grupo $\mathbb{Z}_{2}^{\kappa}$ gerado por r. Como bocks os vértices pertencen a una única órbita segundo $\mathbf{D}_{5}$, cntão, polo Lema 1.4 .2 , os subgrupos de isotropia de todos os vértices são conjugados. Se tomamos $0 \neq t \in \mathbb{R}$, então $\Sigma_{l z}=\Sigma_{z}$, ou seja, todos os pontos exceto a origen que pertencem às retas que passan por un vértice a pela origen têm mesmo subgrupo de isotropia.

Consideramos agora um ponto próximo do eixo real, mas nãa no eixo, indicado por - na Figura 1.1. Se aplicamos $\kappa$ ou $\xi^{j}$, para $j=0,1,2,3,4$, obtemos mar órbita com $2 n-10$ elementos, mass a únice nemento do grupo $\mathbf{D}_{5}$ que fixa estes á a identirlake. Portanto, codos os elenentos que estão entre as retas vórtice-origem pertencem a um mesmo tipo de órbita. 


\begin{tabular}{|c|c|c|}
\hline Tipo do órbita & Subgrupo de isotropia & Tamanho da órbita \\
\hline$\{0\}$ & $\mathbf{D}_{n}$ & 1 \\
\hline$\left\{z \in \mathbb{C} ; \operatorname{Im}\left(z^{n}\right)-0, z \neq 0\right\}$ & $\mathbb{Z}_{2}$ & $n$ \\
\hline$\left\{z \in \mathbb{C}_{i} \operatorname{lm}\left(z^{n}\right) \neq 0\right\}$ & 1 & $2 n$ \\
\hline
\end{tabular}

Tabela 1.1: Tipos de óbitas e subgrupos de isotropia do grupo $\mathbf{D}_{n}$ agindo en $\mathbb{C}$ com $n$ inil)al

Finalmente, a origem forma ela própria uma órbita c com subgrupo de isotropia todo $D_{r_{1}}$. Portanto, $\mathbb{C}_{\text {possui }} 3$ tipos de órbitas pela argào de $\mathbf{D}_{5}$. Na labula 1.1 listannos os lipos de órbitas parta $n$ impar geral.

No caso cuando $n$ ó par, os pontos pertencentes às retas que ligan a origem com o ponto médio enere os vértices possuen subgrupos de isotropia näo triviais o nấo conjugados àueles listados na Tabola 1.1.

Vimes neste exemplo que quanto maior a órbila, menor o subgrupo de isotropia. Formalizanos esta observação como segue:

Proposição 1.4.3 Seju $\Gamma$ um grupo de Lie compacto agindo em V.

a) Se $|\Gamma|<\infty$, entäo $|\Gamma|=\left|\Sigma_{x}\right||\Gamma x|$.

b) $\operatorname{dim} \Gamma=\operatorname{dim} \Sigma_{i}+\operatorname{dim} \Gamma x$

Observação 1.4.4 - A proposição anterior nos diz que a ordem de $\Gamma$ é o produto da ordem de $z_{x}$ pelo tamanho da órbita de $x$, I’x.

- Grupos de Lie sĩo sempre variedades suaves e tem dinemsáo bem dofinda. Assim, subgrupos de isotropia são subgrupos de Lie, e ambos dim $\Gamma$ e dim $\Sigma_{x}$ estão ben definidos. Similarmente, órbitas de grupos de Lie são sempre subvariedares o possuem dimensoes ben definidas.

Demonstração (Proposição 1.4.3) Considere a aplicação natural

$$
\begin{aligned}
\varphi: \Gamma & \rightarrow \Gamma x \\
\gamma & \mapsto \gamma x .
\end{aligned}
$$

Notanos que $\varphi$ é sobrejetora en $\Gamma x$ e $\varphi^{-1}(x)=\{\gamma \in \Gamma ; \varphi(\gamma)=x\}=\Sigma_{1}$. Definamoso subespaco cuociente de um subgrupo $\Sigma$ de $\Gamma$ por

$$
\Gamma / \Sigma=\{\gamma \Sigma ; \gamma \in \Gamma\}
$$

Dircmos que os quocientes de $\Sigma$ em $\Gamma$ sào os conjuntos

$$
\gamma \Sigma=\{\gamma \delta ; \delta \in \Sigma\}
$$

A aplicaçầ $p$ intu\% mma aplicação bijetora

$$
\begin{aligned}
& \psi: \Gamma / \sum_{x} \rightarrow \Gamma x \\
& \gamma \quad \mapsto \dot{\psi}(\gamma)=\gamma x
\end{aligned}
$$


$\log 0$

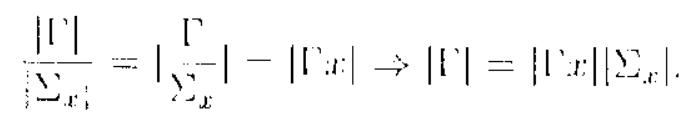

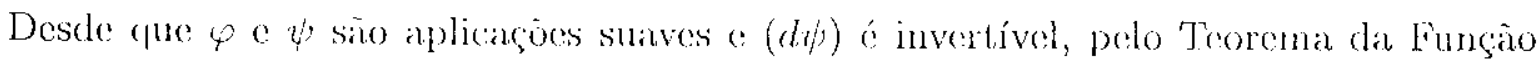

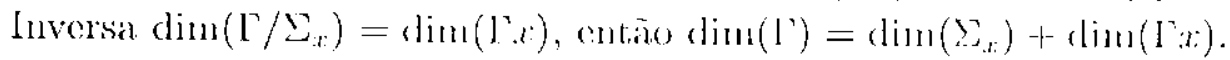

\subsubsection{Subespaço de ponto fixo}

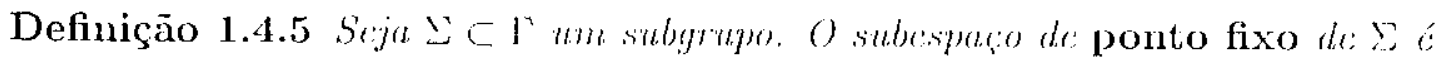

$$
F(X):-\{x \in V ; \sigma x=x, \vee 0 \in \Sigma\} \text {. }
$$

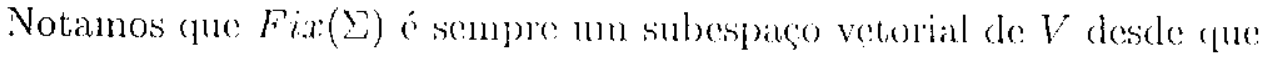

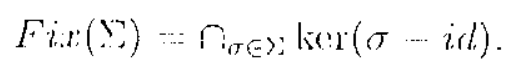

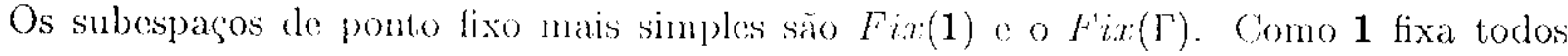
os elementos de $V$, então $F i x(\mathbf{1})=V$. Por outro laxlo, $F i x(\Gamma)$ consiste de todos os elementos due sĩo fixades por $\Gamma$, ou seja, é o subesparco em cuue $\Gamma$ age trivialmente. Em nosso trabalho, assminimos (jue $\operatorname{Fin}(\mathrm{\Gamma})=\{0\}$.

Verificanos que os subespaços de ponto lixo tem a propriedade de invariancia por uma

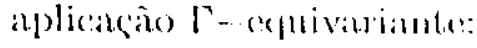

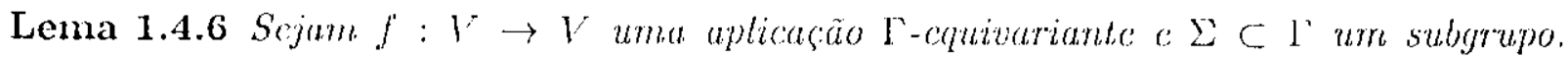
Então,

$$
f(F i x(\Sigma)) \subseteq H^{\prime} i x(\Sigma)
$$

Demonstraçāo Sejann $\sigma \in \sum$ e $x \in F i x(\Sigma)$. Entĩo,

$$
f(x)=f(\sigma x)=\sigma f(x),
$$

$\sigma$ fixa $f(x)$, daí, $f(x) \in F \nmid x(\Sigma)$.

No lema acima não exigimos $\Sigma$ um subgrupo de isotropia. Contudo, Fix( $\Sigma)$ é uma

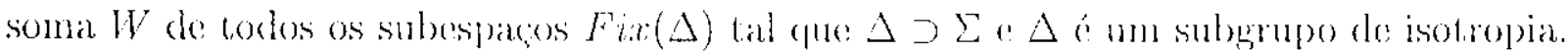
De fato, seja $x \in F^{\prime} i x(\Sigma)$ o $Z_{x}-\{\gamma \in \Gamma ; \gamma x=x\}$ seu subgrupo de isotropia, ontäo $2 \subset \Sigma_{x}$ e $x \in F i x\left(\Sigma_{x}\right)$. Desde que tomanos $\Delta=\Sigma_{x}$,

$$
x \in \operatorname{Pix}(\Sigma) \subset \operatorname{Pix}\left(\Sigma_{n}\right) \subset W .
$$

Logo,

$$
F x(\Sigma) \subset W \text {. }
$$

Reciprocamente, se $w \subset W$ e $w=w_{1}+\cdots+w_{k}$, onde $w_{j} \in F i x\left(\Delta_{j}\right) \circ \Delta_{j} \supset \sum$ subgrupo de

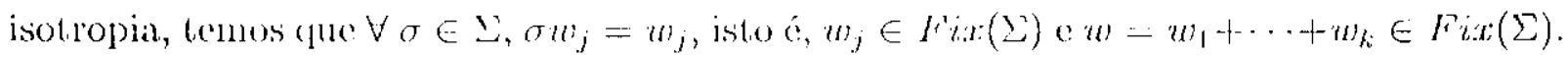
Concluímos cque Fix (¿) $=W$

Cono consequencia do Lema 1.4 .6 temos due se fix $(\Gamma)-\{0\}$ entäo zero tem que ser

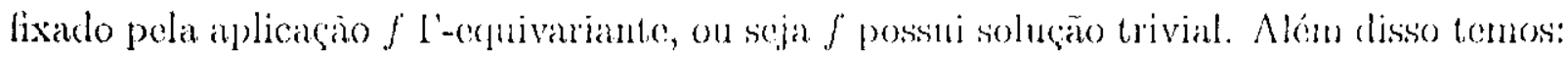


Proposiçāo 1.4.7 Seja $\Gamma$ um grupo de Lie compacto agindo cu V. As seguintes afirmacoues sibo equivalentes:

a) $\operatorname{Hir}(\mathrm{l})=\{0\}$

b) Toda aplicascio $\Gamma$-equmariante $f: V \rightarrow V$ satis $f a z f(0)=0$

c) A minaca funçao r-inuariunte é ufunçao nula.

Demonstração $\quad$ a) $\Rightarrow$ b) Mostramos anterionmente.

b) $\Rightarrow$ a) $\operatorname{Sejan} v \in F i x(\Gamma), \gamma \in \Gamma$ e $f: V \rightarrow V$ a apticacào constante $f(x)=v$. Notamosis (1u0

$$
\gamma f(x)=\gamma u=v=f(\gamma x)
$$

ou seja, $f$; $\Gamma$-equivariante. Agora, por hipótese temos $0=f(0)=v$. Como isto vale para qualduex $v \in F i x(\Gamma)$, Fix $(1)=\{0\}$.

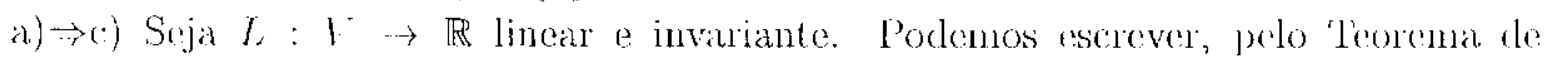
Representaça de Riesz (ver $[11]), L(x)=\langle v, x\rangle_{\Gamma}$ para algum $v \in V$. Afirmagà $v \in$ Fix (T). Logo, $L(x)=\langle v, x\rangle_{\Gamma}=\langle 0, x\rangle_{\Gamma}=0$. Portanto, $L \equiv 0$.

Prova da alfirmagà como $L$ é invariante então $\forall \gamma \in \Gamma, L(x)=L\left(\gamma^{-1} x\right)$. Futäo, $\langle u, x\rangle_{1}=\left\langle v, \gamma^{-1} x\right\rangle_{1}=\langle\gamma u, x\rangle_{1}$ para todo $x \in V$ e $\gamma \in \Gamma$, clá, $\gamma v-v$, on seja, $v \in$ $F(\Gamma)=\{0\}$.

c) $\rightarrow$ b) Scja $f: V \rightarrow$ C equivariante Queremos mostrar (que $f(0)-0$. Para isto definimus $L: Y \rightarrow \mathbb{R}$ tal que $L(x)-\langle f(0), x\rangle$, onde $\langle$,$\rangle o um produto intermo invariante,$ isto a possível pela Proposiçãol.1.12. Afrmamos que a funçào $L$ é invariante. Se isto ocorre entäo $L \equiv 0$ e $\int(0)=0$. Para verificar a afirmação calculamos $L(\gamma x)=\langle f(0), \gamma x\rangle=$ $\left\langle\gamma^{-1} f(0), x\right\rangle=\langle f(0), x\rangle=L(x)$. Portanto, $L$ ć invariante e segne cues $f(0)=0$.

A seguir temos un dos principais resultados do nosso traballo. Mas antes introrluzimos duas definicóose que facilitarão nos enunciados dos lemas o teoremas.

Definição 1.4 .8 Seju $\urcorner$ um grupo de Lie agindo em $\downarrow$. Um subgrupe $\Sigma \varsigma_{-} \Gamma$ é axial se ele é um subyrupo de isotropia tendo subespaco de ponto firo de dimensiono um. Em simbolos, $\operatorname{dim}(F i x)=1$.

Uma outra classe de subgrupos de isotropia ć apresentada na próxima defiugăo.

Definiçāo 1.4.9 Seju $\Gamma$ um grupo de Lie agindo em $\mathrm{V}$. Un subgrupo isotropia $\Sigma \subset \Gamma$ é maximal se näo existe um subgrupo de isolropia $\Delta$ de $\Gamma$ satisfazendo $\Sigma \nsubseteq \Delta \nsubseteq \Gamma$.

Observação 1.4.10 - Sojan $\Sigma$ e subgrupos de isotropia da $\Gamma$. So $\Sigma \subset \Delta$ entaío

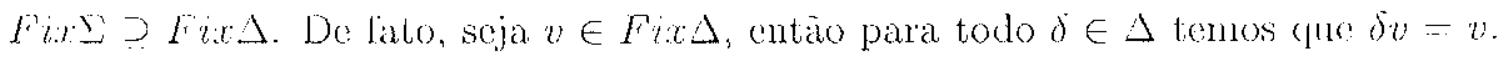

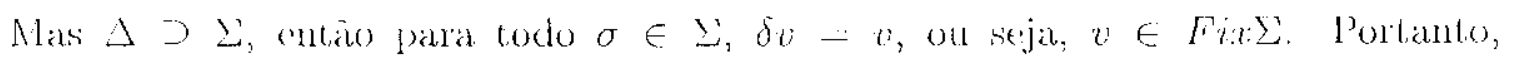
Fix $\supset$ Fin $\triangle$. 
- Se $\Sigma$ é un subgrupo axial segue que $\Sigma$ ć um subgrupo do isotropia maximal. De fato, suponhames (que o subgrupo axial 2 não é subgrupo do isotiopia maxinal. Então, existe un subgrapo de isotropia $\Delta$ tal que $\Gamma \geq \Delta \supseteq \Sigma$. Como dim $F x=1$, então Fix $\Delta q F i x \Sigma$ e dim Fix $\Delta=0$, o que ó uma contradição, pois $\Delta$ ć um subgrupo de isotropia. Portinto, $\mathbf{Z}$ e mu subgrupo de isotropia maximal. Verificanos que se

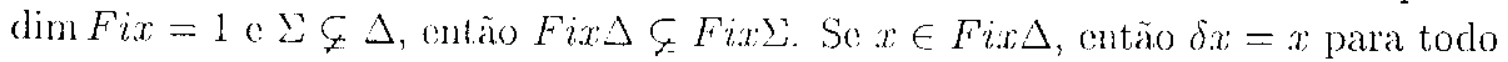

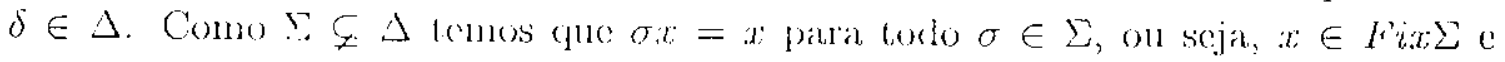

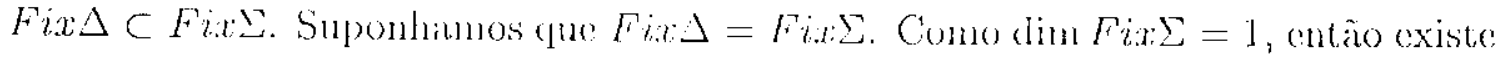
$w_{0} \in V$ não nulo dal que $F$ ix $\Sigma=\left[w_{0}{ }^{3}=F\right.$ ix $\Delta$. Além disso, $\Sigma$ c $\Delta$ são subgrupos de isotropia, enliue existem $x_{0}, y_{0} \subset V$ uăo mulus, onde $\Sigma=\Sigma_{x_{0}}$ e $\Delta=\Sigma_{y_{0}}$. Daí,

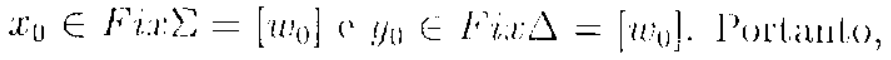

$$
\Sigma=\Sigma_{n_{0}}=X m_{0}-\Delta
$$

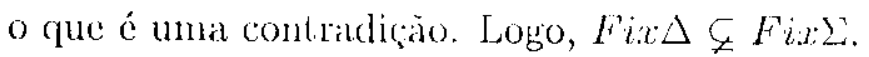

\subsubsection{Lema dos Ramos Equivariantes}

Apresentanos aqui 16 resultado devido a Vanderbauwheds [1980] e Cicogna [1981] que garante sob certas condiçôes no subgrupo de isotropia $\Sigma$ existência um único ramo de

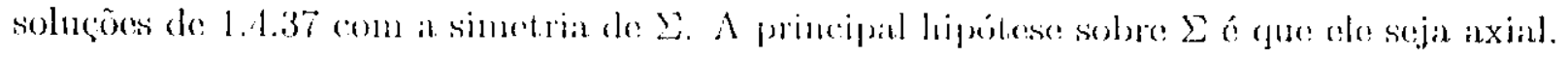

Observação 1.4.11 Denolannos $\varepsilon_{x, \lambda}$ o espaço dos gernes $g: \mathbb{R}^{n-\mu} \rightarrow \mathbb{R}$ em unna vizinhança da origem, onde $x \in \mathbb{R}^{n}$ é a variável padrão o $\lambda \in \mathbb{R}$ c o parâmetro de bifurcação de 1.4.37. De mancina análoga, $\varepsilon_{\lambda}$ é o espaço dos germes das funções de $\mathbb{R}$ em $\mathbb{R}$ na variável $\lambda$.

Definiçāo 1.4.12 Seja l' um grupo de Lie agindo em um espaço velorial V. Um problema de bifurcaşão com grupo de simetria $\Gamma$, on $\Gamma$-equivariante, á un germe $y \in \overrightarrow{\mathcal{E}}_{x, \lambda}(\Gamma)$ salis fazculo $\left.y(0,0)=0 \varepsilon\left(d_{y}\right)\right)_{0,0}=0$.

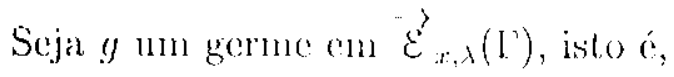

$$
g: V \times \mathbb{R}, 0 \rightarrow V
$$

Saltisfazcndo

$$
g(\gamma x, \lambda)=\gamma g(x, \lambda)
$$

para lodo $\gamma \in \Gamma, x \in V \quad$ e $\lambda \in \mathbb{R}$. Cono fix $x\left(\Gamma^{2}\right)=\{0\}$, então pela Proposição 1.4 .7 , $g(0, \lambda)=0$ para todo $\lambda \in \mathbb{R}$. Daí, $g(0,0)=0$. Exigimos também que $(d g)_{0,0}=0$.

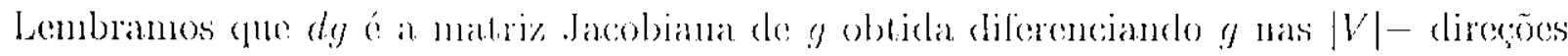

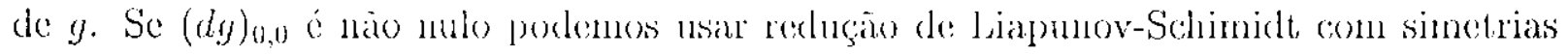
para reduzir $g$ ao caso em que o Jacobiano se anula. A redução de Liapunov-Schmidt

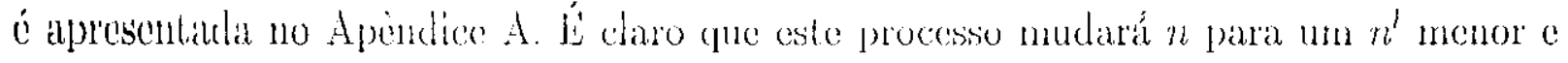


também mudará a representação de $\Gamma$, mas este processo preserva as simetriats. Assinn, assumimos que esta redução já temlua sido feita e, portanto, $(d g)_{0,0}=0$.

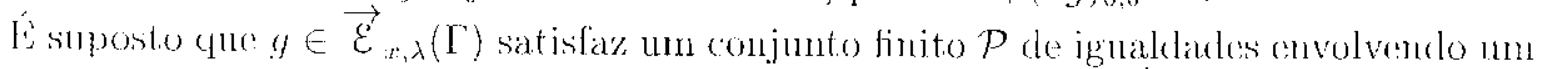

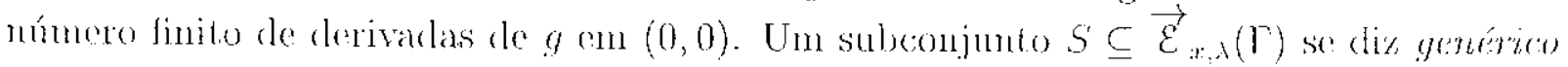
para $\mathcal{P}$ se $g \in S$ satistaz as igualdades em $\mathcal{P}$ o um conjunto finito de desigualdades que chvolven un conjunto finito de derivalas de g na origen que näo contrarlizem as iguaklikles de: $\mathcal{P}$.

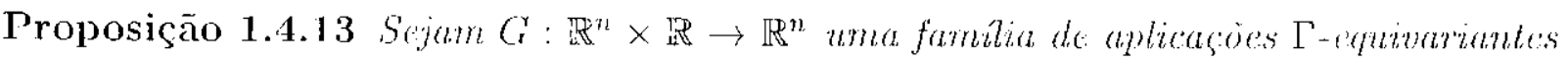
a um parametro com $G(0,0)=0$ e $V=\operatorname{ker}(d G)_{0,0}$. Entäo, genericanente, a açăo de $\Gamma$ em 1 é absolubanente irredutivel.

A Proposiçáco 1.13 dá suporte à nossa última hipólese de que $\Gamma$ age absolutanente ir-

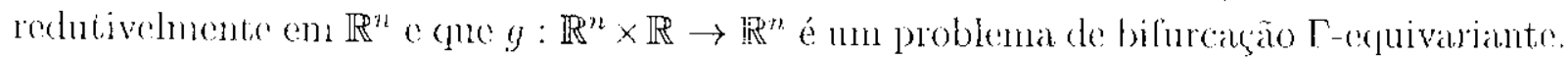
Usamos a hipotese da ação do $\mathrm{T}$ ser absolutamente irredutível como segue. Diforenciando $(1.1 .12)$ com relacar a $x \operatorname{cm}(x, \lambda)=(0, \lambda)$, temos

$$
(d g)_{0, \lambda} \gamma=\gamma(d g)_{0, \lambda}
$$

para torlo $\gamma \in \mathrm{I}$. Conto $\Gamma$ é absolutancute irredutivel, então as únicas matrizes que commtatur com $\Gamma$ săo als múltiplas da identidade; daí, $(d y)_{0, \lambda}=r(\lambda) /$ com $c \in E_{\lambda}$. Docsede (que $(d g)_{0,0)}-0$ temos $(0)=0$. Agora assmmimos que

$$
c^{\prime}(0) \neq 0 \text {. }
$$

Teorema 1.4.14 (Lama dos Ramos Equivariantes) Seja $\Gamma$ um grapo de Lio am açä -

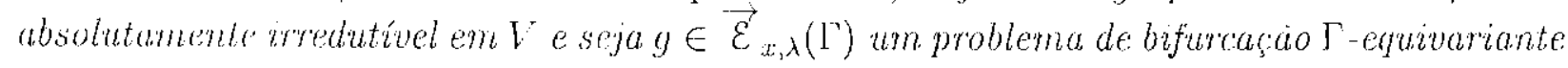
salisfarendo (1.4.44). Seja $\Sigma$ um subgrupo axial. Entaio txiste un unico ramo suave de solurges para $g-0$ tal que o subgrupo de isotropia de toda soluçäo é $\Sigma$.

Podemos resscrever o Lema dos Ramos Equivariantes como segue: Genericamente, problemas de bifurcaça com grupo de simetria $\Gamma$ têm soluçōes correspondentes a todos

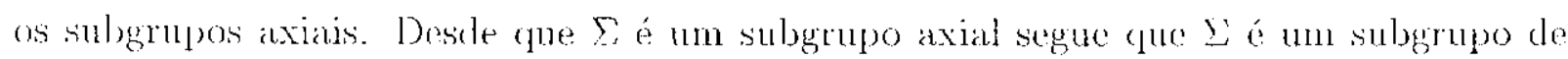
isotropia maximal. Assim, o Lema dos Ramos Equivariantes nos dá um método para encontrar soluges correspondentes a uma classe especial dos subgrupos de isotropia maximais.

Teorema 1.4.15 Seju I" um grupo de Lie agindo em $\mathrm{V}$. Assuminos

a) $P \ln (1)-\{0\}$

b) $\Sigma \subset \Gamma$ um sublytupo axial

c) $y: \checkmark \times \mathbb{R} \rightarrow V$ um problema de bifurcacäo $\Gamma$-equivariante satisfazendo

$$
\left(\lg g_{\lambda}\right)_{0,0}\left(x_{0}\right) \neq 0
$$

onde $x_{0} \in F i x(\Sigma)$ é năo nulo. 
Entäo existe um ramo suave de soluços $\left(t x_{0}, \lambda(l)\right)$ para a equaçäo $g(x, \lambda)=0$.

As observaçòs a seguir mostran que o lema dos Ramos Equiviriantes segue do Teorema 1.4.15.

Observação 1.4.16 1. Açòs irrediltíveis nàu triviais satisfazem $F i x\left(\mathrm{~T}^{\mathrm{T}}\right)=\{0\}$. De fato, como a ação de $\Gamma$ ć irredulível e $F i x \Gamma$ é $\Gamma$-invariante, então $F i x(\Gamma)=V$, ou

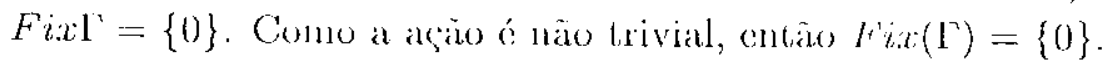

2. Quando a ação de $\Gamma$ em $V$ é absolutanente irredutível, vimos anteriormente que

$$
(d g)_{0, \lambda}-c(\lambda) I
$$

Logo,

$$
\left(d g_{\lambda}\right)_{0,0}\left(m_{0}\right)-K r(0)
$$

onde $K$ é un velor constinte não nulo. Por $(1.4 .45), c^{\prime}(0) \neq 0$. Assim, (1.4.44) equivalente a $(1.4 .45)$.

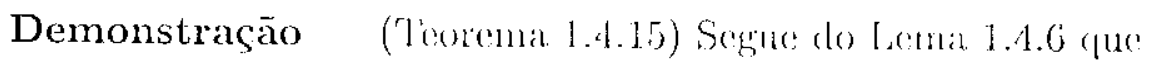

$$
y: \operatorname{lix}(\Omega) \times \mathbb{R} \rightarrow \operatorname{lix}(\Sigma)
$$

Como dim Fix $(\mathrm{Q})=1$ temos que fix $(\Sigma)=\left\{x_{0}, l \in \mathbb{R}\right\}$ para $x_{0} \neq 0, x_{0} \in$ Fix $(\Sigma) \mathrm{c}$ existe $h: \mathbb{R} \times \mathbb{R} \rightarrow \mathbb{R}$ tal que

$$
g\left(t x_{0}, \lambda\right)=h(l, \lambda) x_{0}
$$

Com a hipótese Fix $\left(1^{\prime}\right)=\{0\}$ pela Proposiçâo $1.4 .7 g(0, \lambda)=0$. Airıda,

$$
g(0, \lambda)=0 \Leftrightarrow h(0, \lambda) x_{0}=0 \Leftrightarrow h(0, \lambda)=0 .
$$

Pelo Teorema de Taylor (em torno de $\ell=0)$ temos

$$
h(t, \lambda)=t k(t, \lambda)
$$

tal que $k: \mathbb{R} \times \mathbb{R}, 0 \rightarrow \mathbb{R} e g\left(t x_{0}, \lambda\right)=k(t, \lambda) t x_{0}=k(0, \lambda)\left(l x_{0}\right)+\cdots$. Como $(d g)_{0, \lambda}=$ $c(\lambda) I_{n \times n}$ em que $c(0)=0 e c(0) \neq 0$, temes que $c(\lambda)=h(0, \lambda)$ em que $k(0,0)=0 c$ $k_{\lambda}(0,0) \neq 0$. Pelo Teorema da Função Implícita, existe é énica $\Lambda: U \subset \mathbb{R} \rightarrow V$ tal que

$$
l(l, \Lambda(l))=0 \text { : } \Lambda(0)=0
$$

Logo,

$$
g\left(l x_{0}, \Lambda(l)\right)=\underbrace{l(t, \Lambda(t))}_{=0}\left(x_{0} \equiv 0\right.
$$

Portanto, $\left(t x_{0}, \Lambda(t)\right)$ é $1 m$ ramo de soluçöes de $y=0$.

Consideramos $\Gamma^{\prime}=\mathbf{D}_{n}$ e $V=\mathbb{C}$. Sabemos que o subgrupo de isotropia de todo ponto no cixo real é o subgrupo $\mathbb{Z}_{2}^{r}$ com dois clementos gerado por $\kappa_{i}: z \mapsto \bar{z}$. Mais ainda, os 
únicos clementos em $\mathbb{C}$ fixados por $\kappa$ são os reais. Assin, $F i x\left(\mathbb{Z}_{2}^{\kappa}\right)=\mathbb{R}$ e $\operatorname{dim} F i x\left(\mathbb{Z}_{2}^{\kappa}\right)=1$. Concluímos, usando o Lema dos Rannos Espuivariantes que, genericanente, problemas de bifurcação $\mathbf{D}_{n}$-equivariantes tim ramos de soluçoes consistindo de soluçös com sinctria $\mathbb{Z}_{2}^{\kappa}$.

Seja $x_{0} \in$ Fix 2 tal que $x_{0} \neq 0$. Na prova do Teoremal 1.4 .15 dizemos que um ramo de

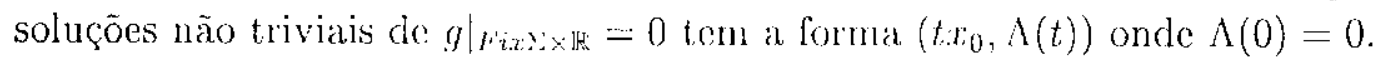

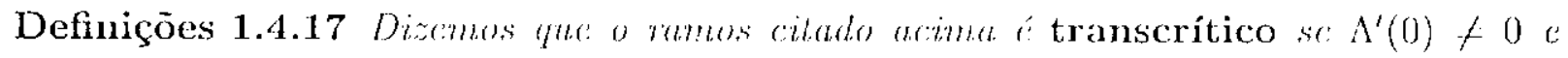
degenerado se $\Lambda^{\prime}(0)=0$.

Enbre os ramos transerticos lomos a scrumbe divisio: o ramo $\left(t x_{0}, \Lambda(l)\right)$ ésubcrítico se, para todo l. nio nulo promimo da origem,

$$
M^{\prime}(l)<0
$$

e é supercrítico se, para lodo t. näo nulo próximo da origem,

$$
t^{\prime}(t)>0
$$

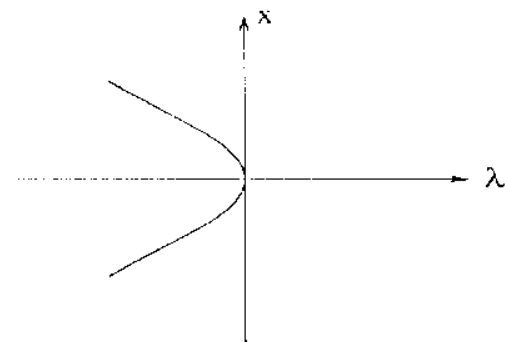

a)

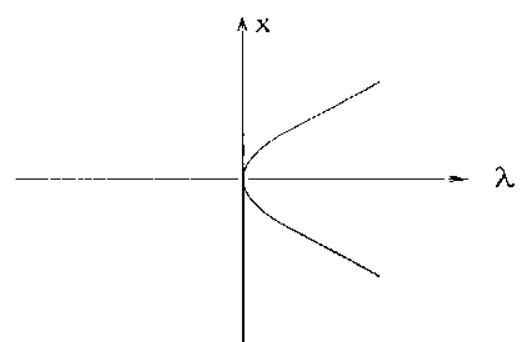

b)

Figura 1.2: Ramos de soluções (a) subcrílico e (b) supercrílico

A Figura 1.2 ihustra um rano de soluçoes subcritico o un supercrítico.

\subsubsection{Estabilidade de soluções}

Estudamos aqui a estabilidade das soluções e introdızinos um novo tipo de estabilidade, a estabilidade orbital.

Consideramos $x_{0}$ un ponto de equilíbrio para un sistema de EDOs

$$
\frac{d x}{d t}+y(x)=0
$$

Definições 1.4.18 1. $x_{0}$ é assintolicamente cslável se loda trajetória $x(t)$ da EDO que inicia próximo de $x_{0}$ permanece próximo de $x_{0}$ para $l>0$ e $\lim _{b}, x_{0} x(l)=x_{0}$.

2. $x_{0}$ neutramente estável se a lrajotória fiea mónimo de $x_{0}$ para todo $t>0$. 
3. $x_{0}$ é instável se sempre existe uma trajetória iniciando próximo de $x_{0}$ que nào permanece prótimo de $x_{0}$ yara $1>0$.

4. $x_{0}$ é lincarmente estanel se lodos os aulovalores da malviz $\left(\right.$ dg) $x_{0}$ lên parte real positiva.

O teorema parleão afirma que se $x_{0}$ ó lincarmonte estável cntão $x_{0}$ é assintoticamente estível. Enn Hirsch o Sinale [197.1] (p.187) temos fue so algum antovalor de (dy) $)_{x_{0}}$ possui parte real negativa, entio xo é instável.

Vimos un ontro tipo de estabilidade fue surge no contexto com simetria, a estabilidade orbital. Sejan $\Gamma$ um grupo de Lie agindo em $V, g: V \rightarrow V$ aplicação $\Gamma$-equivariante, $x_{0}$ um ponto de equilibrio do sistema (1.4.16) o $:=\Sigma_{x_{0}}$ o subgrupo de isotropia de $x_{0}$. Afirmamos que dim $\Omega<$ dim $\Gamma$ inplica que $x_{0}$ năo pode ser assintoticancnte estável. De fato, relembramos da Proposigào 1.4.3b) que dim $\Gamma^{\prime}=\left(\lim \Sigma_{x_{0}}+\operatorname{dim} \Gamma^{\prime} x_{0}\right.$. Com isso temos que a órbita $\Gamma x_{0}$ é una subvariedade de $V$ de dinensão positiva. Assim existe $\gamma \in \Gamma$ tal que $\gamma x_{0} \in \Gamma x_{0}$ é solnção de equilíbrio arlyitrariamente próxino de $x_{0}$. As trajetórias iniciando nestes equilíbrios são fixados por todo tempo não tendendo a $x_{0}$. Assim, $x_{0}$ não é assintolicamente estável. Contudo, $x_{0}$ bode ser neutramente estável. Na verdade, $x_{0}$ pode satisfizer un tipo especifico de estabilidade neutra:

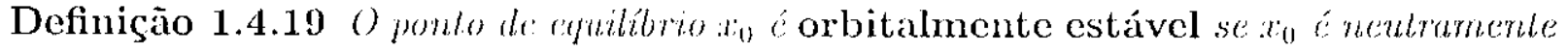

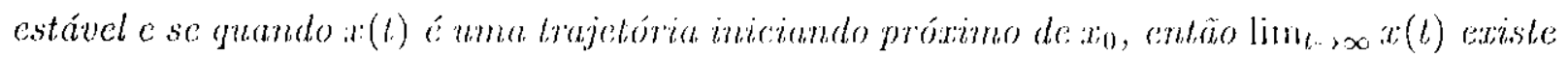
c perlence a rato.

Existe um critério linear para a estabiliclade orbital. Para mostrar isto, primeiro indicamos porque a estabilidade linear falha. Temos que

$$
\operatorname{ker}\left(d_{y}\right)_{x_{0}} \supset I^{\prime} x_{0}\left(\mathrm{~L}_{x_{0}}^{\prime}\right)
$$

onde $T_{x_{0}}\left(\Gamma x_{0}\right)$ denota o espaço tangente de $\Gamma x_{0}$ en $x_{0}$. Segue de (1.4.47) que $(d g)_{x_{0}}$ dever ter autovalor nulo. De lato, $x_{0} \in T x_{0}\left(\Gamma x_{0}\right) \subset \operatorname{ker}(d y)_{x_{0}} \Rightarrow(d g)_{x_{0}}\left(x_{0}\right)=0 \Rightarrow x_{0}$ é autovetor associado ao autovalor nulo. Com isso, nem todos os autovalores de $(d g)_{x_{0}}$ possuem parte real positiva, entĩo a establitidade linear não ó possível en $x_{0}$.

Definição 1.4.20 Seja $x_{0}$ um ponto de equilíbrio de (1.4.46), onde g comuta com a ação de $\Gamma$. O ponto de equilítrio $x_{0}$ é linearmente orbitalmente estável se os autovalores de $\left(d_{g}\right)_{x_{0}}$, diferentes daqueles que surgiram do T $x_{0}\left(l^{2} x_{0}\right)$, têm qurte real positiva.

Em outras palavras, $x_{0}$ é lineamente orbilalunente estável se aqueles antovalores de $(d g)_{x_{0}}$ não forçados pela açio de grupo a serem zero possuen parte real positiva.

Teorema 1.4.21 Estabilidade linear orbilal implica em estabilidade orbilal. 
Como vimos om (1.4.43), dg satisfaz

$$
(d g)_{\gamma x \gamma}=\gamma\left((l g)_{x}\right.
$$

Soja $\Sigma \in I^{\prime}$ o subgrupo de isotropia de $x$. Fudua para todo o $E \Sigma$ a igualdarle (1.4.48) fic $(:)$

$$
(d g)_{x} \sigma=\sigma(d g)_{x}
$$

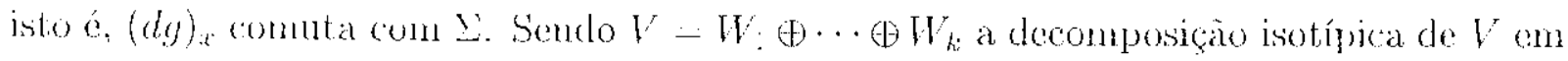

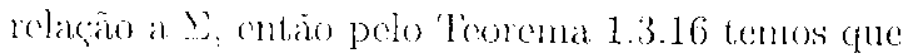

$$
\left(b_{g}\right)_{x}\left(W_{j}\right) \subseteq W_{j}, j=1, \cdots, k
$$

As retrigoss de $(d y)_{x}$ esta frequentenente sujeitas a condigoes extras. Por exem-

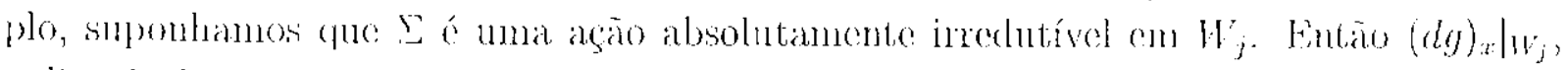
aplicaço linoar que connuta com $\Gamma$, é um múltiplo da identidade.

Agora consideramos dois exemplos: $\mathrm{D}_{n}$ e $\mathrm{O}(2)$ en suas representaços partajo em $\mathbb{C}$.

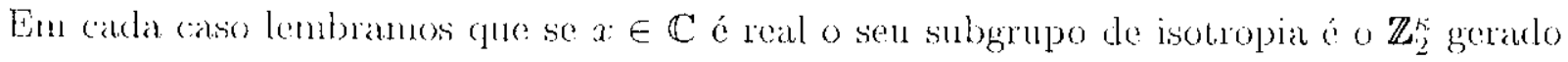
por $f: z a>\bar{z}$. Em coordenadas reais a matriz de $k$ é dada por

$$
L=\left(\begin{array}{cc}
1 & 0 \\
0 & -1
\end{array}\right)
$$

Assim, $W_{1}=\mathbb{R}$ e $W_{2}=i \mathbb{R}$. A açào $L$ em $W_{1}$ é a iclentidade e en $W_{2}$ é menos a iclentidade. As representaçoes săo listintas e absolutannente irtedntíveis.

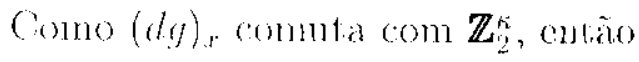

$$
(d g)_{x}=\left(\begin{array}{cc}
a & 0 \\
0 & d
\end{array}\right) ; a, d \in \mathbb{R}
$$

No caso $\mathrm{I}^{\prime}=\mathrm{D}_{n}$, a forma (1.4.51) é tudo o que podemos dizer. Quando $\mathrm{\Gamma}=\mathrm{O}(2)$ tentos que $y(t)=\gamma(t) x \in \mathrm{O}(2) x, \operatorname{com} \gamma(t)=\left(\begin{array}{cc}\cos t & \cdots \operatorname{sen} t \\ \operatorname{sen} t & \cos t\end{array}\right)$. Descle que $x$ é ponto de equilibrio e gé $\mathrm{O}(2)$ - couivariante

$$
g(y(t)) \equiv 0
$$

Daí;

$$
0-\left.\frac{d}{d l} g(y(t))\right|_{t=0}-\left(\begin{array}{ll}
a & 0 \\
0 & d
\end{array}\right)\left(\frac{d}{d l}\left(\begin{array}{cc}
\cos t & -\operatorname{sen} t \\
\operatorname{sen} t & \cos t
\end{array}\right)_{t=0}\left(\begin{array}{l}
1 \\
0
\end{array}\right)\right)=\left(\begin{array}{ll}
a & 0 \\
0 & d
\end{array}\right)\left(\begin{array}{l}
0 \\
1
\end{array}\right) .
$$

Portanto, $d=0$

$$
(d y)_{x}=\left(\begin{array}{cc}
a & 0 \\
0 & 0
\end{array}\right) ; a \in \mathbb{R}
$$

Enfatizanos que usando a estrutura de simetria de g pudemos obter (dy).x sem de fato calcular an derivalas da $g$ explicitamente. 


\section{Capítulo 2}

\section{Sistemas de células acopladas com simetria do tipo produto coroa}

'Tratamos neste capítulo da parte central do projeto: bifurcaçĩo de pontos de exuilíbrio em sistemas acoplados em presenga de simetria.

Discutimos a estrutura de sistemals de cólulas acopladas como sistema de equaçóes diferenciais ordinárias onde simetrias do sistema são obtidas através do grupo $\mathcal{G}$ das permutações das cólulas - chamadas simetrias globais do sistema - e do grupo $\mathcal{L}$ das simetrias internas de cada colula - chamadas simetrias internas. As simetrias globale interna podem ser combinaklisis de duas maneiras, flependendo de como a simetria interna afeta o acoplamento. Algebricanente, estas duals combinaçoes são danlas pelo "produto coroa" $\mathcal{L}$; $\mathcal{G}$ un pelo "produto direto" $\mathcal{L} \times \mathcal{G}$. Os nossos estuclos sĩo clirecionimbs para a teoria do produto coroa.

O produto coroa ocorre quando o acoplamento é invariante sob a ação do grupo de simetrias internats. O principal objetivo é relacionar bilurcaçöes de pontos de equilíbrio

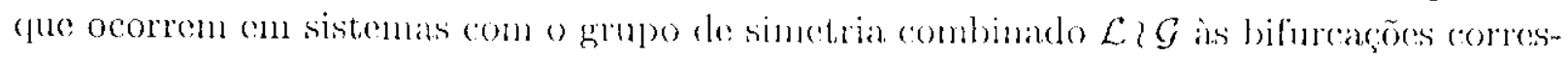
pondentes em sistemas com simetria $\mathcal{L}$ ou $\mathcal{G}$.

En tum arcoplanento de células identicats, as simetrias são induzidas pola mancim como as equaçóes diferenciais estão associadas e essass simetrias dependem do tipo exato de acoplamento. O grupo $\mathcal{G}$ das simetrias globais é sempre um subgrupo findito de $\mathrm{S}_{N}$. Por

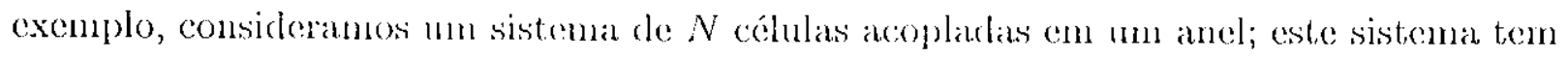
a simetria do grtupo diedral $\mathrm{D}_{N}$. Unt outro exemplo é o acoplamento "dois a dois" onde cada célula esta acoplada a todas as outras. Hste tipo de acoplamento leva à simetria do grupo das permutacoos $\mathrm{S}_{N}$.

O conjunto das simctrias internas, por sua ve\%, ocorre quando as equaçoes diferenciais que governam a clinamica do cada cóluba possuom sla própria simetria e a dinâmica

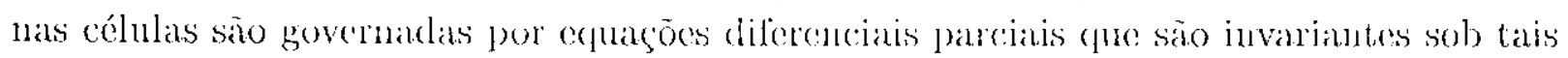
simetrians.

Estudanos a seguir propriedades que o acoplamento deve possuir quando as simetrias internas e globais combinadas forman o produto coroa. $\Lambda$ partir destas propriedade podenos obter uma forma geral para um sistema com simetria produto coroa. 


\subsection{EDOs de sistemas acoplados com simetria do tipo produto coroa}

Inicianes com a forma de aceplamento geral de sistemas de EDOs que pessinem a hipótese de células irinticas com acoplamento identico; esta forma de aroplamonto permibirá ilustramos conno o tipe de acoplanento mudar o tipo de sinnetria.

Sejan $Y_{j}$ a variável de ostado da $j$-ésima cólula e $Y=\left(X_{1}, \ldots, X_{N}\right)$ a variável de estado do unn sistema com $N$ células. A hipótese de que as cólulas são identicas implica que $X_{j} \in \mathbb{R}^{k}$ para cala $j$ a $X \subset\left(\mathbb{R}^{k}\right)^{N}$. Nosso objotivo é estudar ramos de solugoes de pontos de enpuilínio de un sistena da forma

$$
\frac{d X}{d t}=F(X)
$$

on seja, sistema de célulis aceopladas

$$
\frac{d X_{j}}{d t}=f_{j}\left(X_{j}\right)+h_{j}(X)
$$

onde $f_{j}$ gorema a dinâmica interna da j-ésima célula $o h_{j}$ governa o acoplamento entre as cólulas paral $j-1, \ldots, N$. Descle que consideramos as cólulas identicas, assmuninos que $f,-f$ pilat $j=1, \ldots, N$.

Para formular nossa hipóteso sobro acoplamento, definimos a matriz de conexão $C$ por

$$
C(i, j)=\left\{\begin{array}{cc}
1 & \text { se a célula i está acoplacla à célula } j \\
0 & \text { (aso contrário. }
\end{array}\right.
$$

Para continum motivando a idéia, assuminos cue o acoplanento ten a forma

$$
h_{j}(X)=\sum_{i=1}^{N} C(i, j) h_{i j}\left(X_{i:} X_{j}\right)
$$

onde hij moclela o acoplamento das células $i$ e $j$. Isto é. consideramos que o efeito do arophlanento na j-ísina célula é encontrado somando as influencias de todas as células

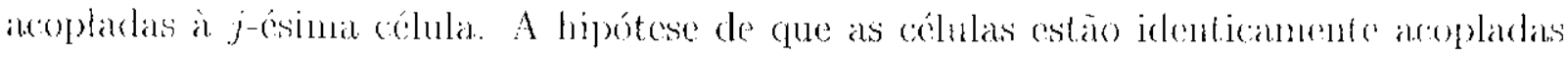
implical gue $h_{\imath}=h, i, j=1, \ldots, N$.

Assim, o sistema (2.1.1) é do tipo

$$
\left(\begin{array}{c}
d X_{1} / d t \\
d X_{2} / d \\
\vdots \\
d X_{N} / d t
\end{array}\right)=\left(\begin{array}{c}
f\left(X_{1}\right) \\
f\left(X_{2}\right) \\
\vdots \\
f\left(X_{N}\right)
\end{array}\right)+\left(\begin{array}{c}
\sum_{i=1}^{N} C(i, 1) h\left(X_{i}, X_{1}\right) \\
\sum_{i=1}^{N} C(i, 2) h\left(X_{i}, X_{2}\right) \\
\vdots \\
\sum_{i-1}^{N} C(i, N) h\left(X_{1}, X_{N}\right)
\end{array}\right)
$$

Discutimus a seguir as simetrias globais presentes neste sistema. Seja $\sigma \in \mathrm{S}_{N}$ uma

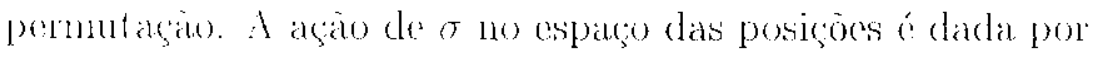

$$
\sigma \cdot X=\left(X_{O^{-1}(1)}, \ldots, X_{\sigma^{-1}(N)}\right)
$$


Agora $\sigma \in \mathrm{S}_{N}$ á mua simetria do sistema (2.1.2) se, a somente se,

$$
F(\sigma X)-\sigma F^{\prime}(X)
$$

Notamos que

lsto implical que

$$
\frac{d \sigma \mathrm{X}}{d t}=\sigma \frac{d \mathrm{X}}{d t} \Leftrightarrow \sigma^{-1} \frac{d \sigma \mathrm{X}}{d t}=\frac{d X}{d t}
$$

$$
\sigma^{-1}\left(\begin{array}{c}
f\left(X_{\sigma^{-1}(1)}\right) \\
f\left(X_{\sigma^{-1}(2)}\right) \\
\vdots \\
f\left(X_{\sigma^{-1}(N)}\right)
\end{array}\right)=\left(\begin{array}{c}
f\left(X_{1}\right) \\
f\left(X_{2}\right) \\
\vdots \\
f\left(X_{N}\right)
\end{array}\right)
$$

e analogamente para $h\left(X_{i}, X_{j}\right)$. Quinto à matriz de conexão, se identificannos a permutação $\sigma \in \mathrm{S}_{N}$ com numa matriz $N \times N$ notamos que $\sigma$ ó uma simetria do sistema se $\sigma C \sigma^{-1}=C$. Verificanos isto consideranto um sistema de $N$ cólulas o sens possiveis tipos de acoplannento, já que a simetria global depende da forma exata do acoplamento das células, além de que a matriz de mua permutaçào à esquerda troca linhas e à direita troca colunas. En suma, $\sigma$ o una simetria do sistema $(2.1 .2)$ se $\sigma C^{\prime} \sigma^{1}=C$. O grupo das sinctrias globais $\mathcal{G}$ consiste precisannonte destats sinctrias.

Allalisamos agora as restricoes inpostas em (2.1.2) pela açĩo do grupo das simetrias

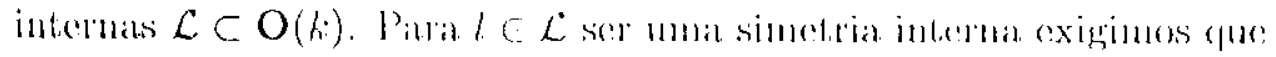

$$
f\left(l X_{j}\right)=l f\left(X_{j}\right)
$$

para $j=1, \ldots, N$. Se simetrias internas sào simetrias de (2.1.2) elas dependem das propriedades do termo de aceplanento $h$. Cono un minimo, exigimos que quando $l$ age

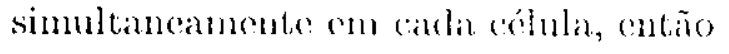

$$
h\left(l X_{i}, l X_{j}\right)=\operatorname{lh}\left(X_{i}, X_{j}\right)
$$

Se delininos

$$
l \cdot X=\left(l X_{1}, \ldots, l X_{N}\right)
$$

entitio

$$
F(l \cdot X)=l F(X)
$$

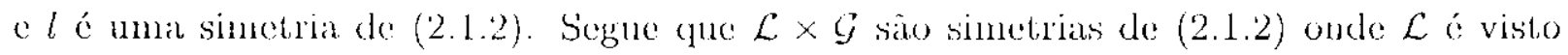
cono o subgrupo diagonal do $\mathcal{L}^{N}$. Contudo, também consideranos sistemas acoplados onde a agão de lem cada célula é umia simetria de (2.1.2). Isto é, supomos que

$$
\begin{aligned}
& h\left(X_{i}, l X_{j}\right)=\operatorname{lh}\left(X_{i}, X_{j}\right) \\
& h\left(l X_{i}, X_{j}\right)=h\left(X_{i}, X_{j}\right)
\end{aligned}
$$

Qualquer duas das equargoss (2.1.3), (2.1.4) c/ou (2.1.5) implica na terceira. Neste caso, o grupo $\mathcal{L}^{N}$ é un grupo de sinctria de (2.1.2). 
O produto coroa $\mathcal{L}<\mathcal{G}$ é un grupo geraelo por $\mathcal{L}^{N}$ e $\mathcal{G}$. Sol estas condições, é um grupo de simetria de (2.1.2). (Ver Observaço 1.1.9, para a definiçäo da aỹo de $\mathcal{L}$ / $\mathcal{G}$ en $\mathbb{R}^{k N}$.)

Un exemplo de acoplanento com simetria do prodato coroa é dado por

$$
h\left(X_{i}, X_{j}\right)=\left|X_{i}\right|^{2} X_{j}
$$

Neste caso, o sistema lica

$$
\frac{d X_{i}}{d t}=f\left(X_{j}\right)+\sum_{i=1}^{N} C(i, j)\left|X_{i}\right|^{2} X_{j}
$$

para $j=1, \ldots, N$.

Definição 2.1.1 $\mathcal{G}$ é um subgrupo transitivo $d e \mathrm{~S}_{N}$ se para quaisquer $i, j \in\{1,2, \cdots, N\}$ existe $\sigma \in \mathcal{G}$ tal gue $\sigma(i)=j$, ou seja, se a açü de $\mathcal{G}$ possuri uma unica ómbita em $\mathrm{S}_{N}$.

Estamos interessartos no tipo de acoplamento que leva à simetria do produto coroal. Para simplifica a análise assmmimos (pue as sinctrias globais agem transitivamente nas

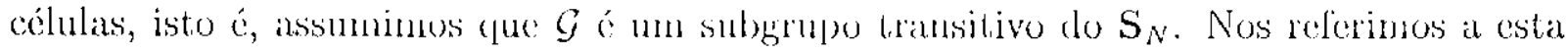
hipótese de transitividiule por $\left(I I_{T}\right)$.

\subsection{Teoria linear do grupo $\mathcal{L} / \mathcal{G}$}

Quando consideramos bifurcaşo de pontos de exuilíbrio a partir de um equilíbrio invariante pelo grupo todo, fazenos a hipótese genérica $\left(H_{S}\right)$ de cue $\Gamma=\mathcal{L} / \mathcal{G}$ age absolutamente irredutivelmente no uncleo das equacoes lincarizadas. Veja Proposicano 1.4.13.

Seja $W \subset V^{N}$ un subespaco l'-irredutível. En particular, $W$ é um subespaço invariante pelo subgrupo $\mathcal{L}^{N}$. Se $\mathcal{L}^{N}$ age trivialmente en $W$, então as sincetrias localis não terão efeito nas bifurcaçoes. Como estamos interessades em estuklan bilurcaçöes con sinnetria local e global associadas, assumimos: $\left(I_{\mathcal{L}}\right) \mathcal{L}^{N}$ age naio trivialment,e em $W$.

$\operatorname{Soga} U_{j}=\left\{v_{j} \in V_{;}\left(0, \cdots, v_{j}, \cdots, 0\right) \in W, j-1, \ldots, N\right\}$. Cada $U_{j} \subset W$ c un subespaço $\mathcal{L}$-invariante. Do liato, se $v_{j} \in U_{j}$, cntão $\left(0, \cdots, v_{j}, \cdots, 0\right) \subset W$. Como $W$ é $\mathcal{L}^{N}$ - invariante, chtao $\left(l_{1} 0, \cdots, l_{j} v_{j}, \cdots, l_{N} 0\right) \in W$ o $l_{j} v_{j} \in U_{j}$.

Lema 2.2.1 Assumimos as hipóleses $\left(H_{T}\right),\left(H_{S}\right) e\left(H_{\mathcal{L}}\right)$. Entäo

a) $U_{j}<\mathcal{L}$-irredutivel.

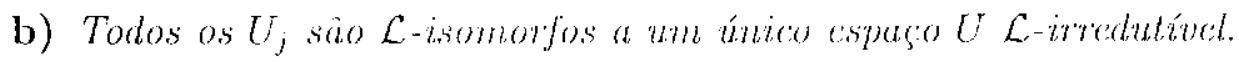

c) $H=U^{N}$ 
Demonstração Por comstiugão, $H^{r} \supset U_{1} \oplus \cdots \oplus U_{N}$. Afimmanos che

$$
\|=U_{1} \oplus \cdots \oplus U_{N}
$$

Notemos (fue $U_{1} \oplus \cdots \oplus U_{N}$ a $\mathcal{G}$-invariante pois $\mathcal{G}$ somente permuta os subespares $U_{j}$. Também por construçầ, $U_{1} \oplus \cdots$ D $U_{N}$ é $\mathcal{L}^{N}$-invariante, pois cada $U_{j}$ é $\mathcal{L}$-invariante. Verilicamos agora que $U_{1} \oplus \cdots \oplus U_{N} \neq\{0\}$. Por hipótese, $\mathcal{L}^{N}$ age não triviahmente em I'. Suponlammos (ule $\left(v_{1}, \ldots, v_{N}\right) \in W$ o $l \in \mathcal{L}$, antäo $\left(l, 1_{L}, \ldots, 1_{L}\right) \in \mathcal{L}^{N}$ "

$$
\left(l, 1_{L}, \ldots, 1_{L}\right)\left(v_{1}, \ldots, v_{N}\right)=\left(l v_{1}, v_{2}, \ldots, v_{N}\right) \in W
$$

Jogo, $\left(l_{1}-v_{1}, 0, \ldots, 0\right) \in W$ para todo $l \in \mathcal{L}$. Por $\left(\mathrm{H}_{\mathcal{L}}\right)$, existe $l$ tall que $\left(v_{1}, \ldots, v_{N}\right) \neq$ $\left(w_{1}, \ldots, w_{N}\right)$ gue, scm perda de generalidade, supôe que $\mathcal{L}$ age näo trivialmente na primeria componente de vetores em $W$. $\log \left(U_{1} \neq\{0\}\right.$ e $U_{1} \oplus \cdots \oplus U_{N} \neq\{0\}$. Conno $W$

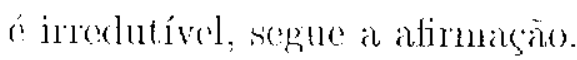

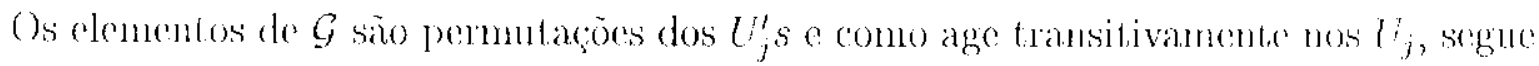
lodos os $U_{j}$ säo $\mathcal{L}$ - isomorfos. Isto $c^{\prime}, U_{j} \simeq U, j=1, \ldots, N$. Agora, so $U_{0} \subset U$ ć $\mathcal{L}$ imedutiven, entao $U_{0}^{N}$ e $\Gamma$-invaliante. $\Lambda$ irredutibilidade de $W-U^{N}$ insplian que $U_{0}-U$ a $U$ : L L-irredutível.

Mostranos no próximo lema que se Firu $\mathcal{L}=\{0\}$, então l' ange absohutamente irre-

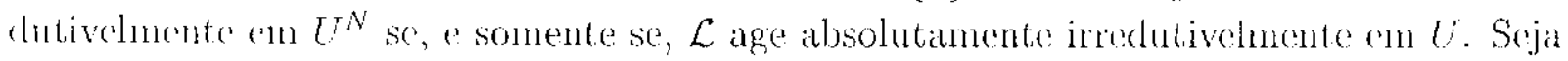
$\mathcal{D}_{1}(11)$ ) espaco das aplicagoes lineares de $W$ em $W$ que comutam con $l$.'

Lema 2.2.2 Suponhamos $\mathrm{\Gamma}=\mathcal{L} \backslash \mathcal{G}$. onde $\mathcal{G}$ é um subgrupo transitivo do $\mathrm{S}_{N}$ e Fixu $(\mathcal{L})=$ $\{0\}$. Entrio,

$$
\mathcal{D}_{1}\left(U^{N}\right) \simeq \mathcal{D}_{\mathcal{C}}(U)
$$

Demonstração Suponhamos que $A: U \rightarrow U$ é uma aplicacão linear pue commuta (om $\mathcal{L}$. Entaio $\mathcal{A}^{N}: U^{N} \rightarrow U^{N}$ comuta com $\mathcal{L}^{N}$ e com $\mathcal{G}$. De fato, para $\left(l_{1}, \ldots, l_{N}\right) \in \mathcal{L}^{N}$

$$
\begin{gathered}
\left(l_{1}, \ldots, l_{n}\right) \cdot A^{N}\left(u_{1}, \ldots, u_{N}\right)=\left(l_{1}, \ldots, l_{n}\right) \cdot\left(A\left(u_{1}\right), \ldots, A\left(u_{N}\right)\right)= \\
=\left(l_{1} \cdot 1\left(u_{1}\right), \ldots, l_{N} A\left(u_{N}\right)\right)=\left(A\left(l_{1} u_{1}\right) \ldots, A\left(l_{N} u_{N}\right)\right)-A^{N}\left(l_{1} u_{1}, \ldots, l_{N} u_{N}\right)= \\
A^{N}\left(\left(l_{1}, \ldots, l_{N}\right) \cdot\left(u_{1}, \ldots, u_{N}\right)\right)
\end{gathered}
$$

e para $o \in S_{N}$

$$
\begin{gathered}
\sigma \cdot A^{N}\left(u_{1}, \ldots, u_{N}\right)=\sigma \cdot\left(A\left(u_{1}\right), \ldots, A\left(u_{N}\right)\right)=\sigma \cdot\left(u_{1}, \ldots, v_{N}\right)=\left(v_{\sigma^{-1}(1)}, \ldots, v_{\sigma}(N)\right)= \\
=\left(A\left(u_{\sigma^{-1}(1)}\right), \ldots, A\left(u_{\sigma^{-i}(N)}\right)\right)=A^{N}\left(\sigma \cdot\left(u_{1}, \ldots, u_{N}\right)\right) .
\end{gathered}
$$

Portanto, $A^{N}$ commla con $\Gamma$.

Reciprocanoute, suponhamos que $B: U^{N} \rightarrow U^{N}$ ó lineir e connta com $\Gamma$. En coorclemalats, seja $B=\left(C_{1}, \ldots, C_{N}\right)$. Então,

$$
\left(l_{1} C_{1}\left(u_{1}, \ldots, u_{N}\right), \ldots, l_{N} C_{N}\left(u_{1}, \ldots, u_{N}\right)\right)=
$$




$$
\begin{gathered}
\left(l_{1}, \ldots, l_{N}\right) \cdot\left(C_{1}\left(u_{1}, \ldots, u_{N}\right), \ldots, C_{N}\left(u_{1}, \ldots, u_{N}\right)\right)= \\
\left(C_{1}\left(l_{1} u_{1}, \ldots, l_{N} u_{N}\right), \ldots, C_{N}\left(l_{1} u_{1}, \ldots, l_{N} u_{N}\right)\right) .
\end{gathered}
$$

Segue que, $C_{j}\left(l_{1} u_{1}, \ldots, l_{N} u_{N}\right)=l_{j} C_{j}\left(u_{1}, \cdots, u_{N}\right)$, para todo $j=1, \ldots, N$. Escolhemos um dos $C_{j}$ 's, digamos $C_{i}$. Por linearidade, podemos escrever, para cada $i=1, \ldots, N$,

$$
C_{i}\left(u_{1}, \ldots, u_{N}\right)=D_{1}^{i}\left(u_{1}\right)+\cdots+D_{i}^{i}\left(u_{i}\right)+\cdots+D_{N}^{i}\left(u_{N}\right),
$$

com $D_{j}^{i}: U \rightarrow U$ linear, $j=1, \ldots, N$. A cquivariancia de $C_{i}$ implica que cada $D_{j}^{i}$ para $j=1, \ldots, N, j \neq i$ ó $\mathcal{L}$-invariante, pois

$$
l_{i} C_{i}\left(u_{1}, \ldots, u_{N}\right)=D_{1}^{i}\left(u_{1}\right)+\cdots+l_{l} D_{\imath}^{i}\left(u_{i}\right)+\cdots+D_{N}^{i}\left(u_{N}\right)
$$

e

$$
C_{i}\left(l_{1} u_{1}, \ldots, l_{N} u_{N}\right)=D_{1}^{i}\left(l_{1} u_{1}\right)+\cdots+D_{i}^{i}\left(l_{i} u_{i}\right)+\cdots+D_{N}^{i}\left(l_{N} u_{N}\right) .
$$

Dari, $D_{1}^{i}\left(u_{1}\right)+\cdots+l_{i} D_{i}^{i}\left(u_{i}\right)+\cdots+D_{N}\left(u_{N}\right)=D_{1}^{i}\left(l_{1} u_{1}\right)+\cdots+D_{i}^{i}\left(l_{i} u_{i}\right)+\cdots+D_{N}^{i}\left(l_{N} u_{N}\right)$. Desde que Fixu $(\mathcal{L})=\{0\}$, segue da Proposição 1.4 .7 que as aplicaçöes lincares $D_{j}^{i}$ para $j=1, \ldots, N, j \neq i$ são mulass. Dái, $C_{i}\left(u_{1}, \ldots, u_{N}\right)=D_{i}^{i}\left(u_{i}\right)$. Assim,

$$
B\left(u_{1}, \ldots, u_{N}\right)=\left(D_{1}^{1}\left(u_{1}\right), \ldots, D_{N}^{N}\left(u_{N}\right)\right)
$$

onde carda $D_{j}^{j}: U \rightarrow U$ comuta com $\mathcal{L}$, para $j-1, \ldots, N$. Como $\mathcal{G}$ é um subgrupo

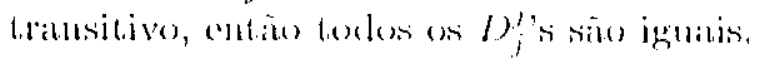

Dos dois lemas Lema 2.2.1 e Lema 2.2 .2 temos o seguinte resultado:

Lema 2.2.3 Assumimos as hipóteses $\left(I_{T}\right),\left(H_{S}\right)$ c $\left(I_{\mathcal{L}}\right)$. Então $\mathcal{L} \backslash \mathcal{G}$ age absolutamente irredutivelmente on $\mathrm{H}$ sc, asomente se, $W \cong U^{N}$ :C age absolulamente irredulivelmente sobre $U$.

O Lema 2.2 .2 inclica a forma dos auto-espacgos críticos nos pontos de explulíbrio: o núcleo da lincarizaçio é genericanente $\Gamma$-absolutianente irredutivel. P’or $\left(H_{T}\right)$ e $\left(H_{\mathcal{L}}\right)$, este núcho deve ter a lorma $U^{N}$ onde $U$ é $\mathcal{L}$-alssolutanneme irredutivel.

\subsection{Quebra de simetria em bifurcação $\mathcal{L} l \mathcal{G}$-equivariante}

Assumimos que $W$ é o núcłeo da lincarização de (2.1.2) em um equilíbrio $\Gamma$-invariante. Com a hipótese genérica $\left(H_{S}\right)$ de que $\Gamma$ age absolutamente irredutivelmente en $W$ e com a hipótese adicional $\left(H_{\mathcal{L}}\right)$ de que $\mathcal{L}^{N}$ age não trivialmonte en $W$, pelos resultados da Seção 2.2 podemos escrever $W^{N}=U^{N}$ onde $\mathcal{L}$ age absolutamente irredutivelmente om $U$ r $\operatorname{rix}_{U}(\mathcal{L})=\{0\}$.

$\mathrm{Na}$ subseção a seguir apresentamos a forna geral dos subgrupos axiais do produto coroa a menos de conjugaciono. 


\subsubsection{Subgrupos axiais de $\mathcal{L} \backslash \mathcal{G}$}

Apresentamos inicialmente algumas notacoies.

Definição 2.3.1 Um subcongunho de indices $f \subset\{1, \ldots, N\}$ é chamado um bloco se: entiste um subyrapo $\mathcal{H} \subset \mathcal{G}$ gue age transitivamente em J.

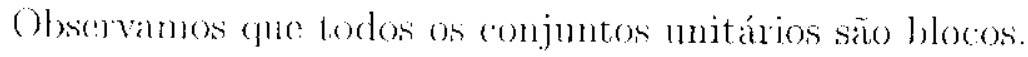

l'ara carda bloco J associamos o subgrupo de permutacós

$$
Q_{j}=\{\sigma \in \mathcal{G} ; \sigma(J)=J\}
$$

que age tramsitivamente em $J$, já que ele contóm $\mathcal{H}$.

Para $A \subset \mathcal{L}$ un subgrupo qualcuer, detinimos o subgrupo

$$
\Sigma(A, J)=\left(B_{1} \times \cdots \times B_{N}\right)+\mathcal{Q}_{j}
$$

ondir

$$
B_{j}=\left\{\begin{array}{l}
A \text { se } j \in J \\
\mathcal{L} \text { se } j \notin J
\end{array}\right.
$$

Lema 2.3.2 P'ara cada bloco J e cada subgrupo axial $A \subset \mathcal{L}$ agindo tem $U$, o subgrapo $\mathrm{Z}(A, J) \subset \mathcal{L}>\mathcal{G}$ ó um subgrupo axial.

Demonstração $\quad$ Srjan $x \in U^{T}$ um vetor não nulo fixado por $A$ o $\mathrm{x}=\left(x_{1}, \cdots, a_{N}\right)$ oncle?

$$
x_{3}=\left\{\begin{array}{l}
x \text { se } j \in J \\
0 \text { se } j \notin J
\end{array} .\right.
$$

Alimagio: $\Sigma(A, J)$ fixa $x$. De fato, $(l, \sigma) \in \Sigma(A, J) \Rightarrow\left(l_{1}, \ldots, l_{N}, 0\right) \in \Sigma(A, J)$ onde $\left\{\begin{array}{l}l_{i \in A} \operatorname{sicj} J \\ l_{i} \in \mathcal{L} \operatorname{sic} j \notin J\end{array}\right.$ e $\sigma \in \mathcal{Q}_{J} \Rightarrow \sigma^{-1}(j) \in J$ se $j \in J$ Logo,

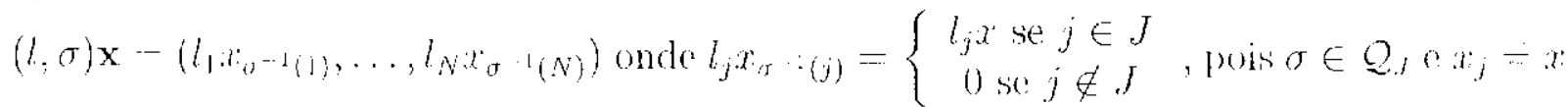
para $j \subset J$. Além disso, temos que para $j \in J, l_{j} \in A$. Como $x$ é tixado por $A$, então $l, x=x$ para $j \in J$. Portanto, $\Sigma(A, J)$ tixa $\mathrm{x}$.

Por ontro lado, seja $\mathrm{y}=\left(y_{1}, \ldots, y_{N}\right) \in U^{N}$ fixado por $\Sigma(A . J)$. Então $y_{j}=0$ se $j \not \subset d$ e $y_{j}$ é un múltiplo de $x$ se $j \in J$. Desde que $\mathcal{Q}_{J}$ age transitivamente en $J$ então torlos os

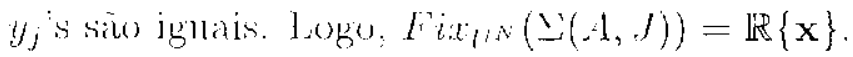

Agora vamos mostrat que $\Sigma(A, J)$ é o subgrupo de isotropia $\Sigma_{\mathrm{x}}$ (le $\mathrm{x}$. Dat alirmariou acima temos que $\Sigma(A, J) \subset \Sigma_{x}$. Suponhamos que $(l, \sigma)$ fixa $x$. isto $\dot{c},(l, \sigma) \in \Sigma_{\mathrm{x}}$. Fntão,

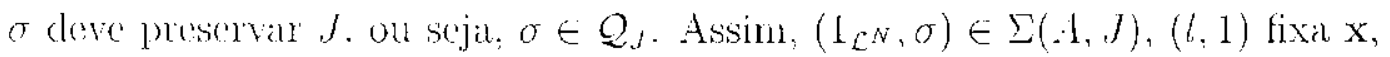

$$
(l, 1)\left(1_{\left.C^{N}, \sigma\right)}=(l, \sigma) \in \mathcal{U}(1, .)\right.
$$

0

$$
\Sigma_{\mathrm{x}} \subset \Sigma(t, J)
$$


Portanto, $\Sigma(A, J)$ ć um subgrupo axial de $\mathcal{L} ? \mathcal{G}$.

Mositrames ma Proposiça 2.3 .4 abaixo que qualquen subgrupo axial do produto coroal é conjugado a um sulogrupo axial da forma $\Sigma(A, J)$.

Sojam

$$
\pi_{\underline{G} ;}: \mathcal{L}>\mathcal{G} \rightarrow \mathcal{G}
$$

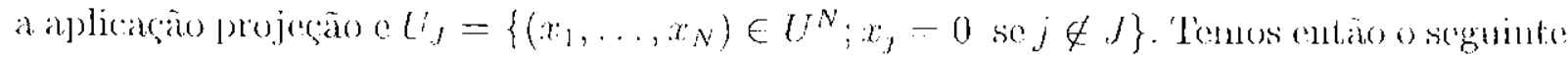
lema:

Lema 2.3.3 Suponhamos $\Sigma$ um subgrupo acial de $\mathcal{L}>\mathcal{G}$. Entôno, $\pi_{\mathcal{G}}(\Sigma)$ age transithounente

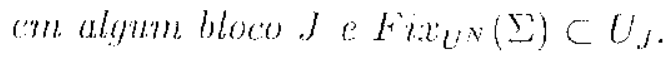

Demonstração $\operatorname{Seja~} \mathrm{x}=\left(x_{1}, \ldots, x_{N}\right) \in F i x(\Sigma)$ näo nulo. Scjal $J \subset\{1, \ldots, N\}$

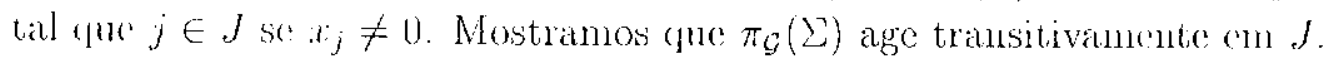

Primeirancente, mostramos que $\pi_{G}(\Sigma) . J \subset J$. Seja $i \in J$. Para qualefuer $\left(l, \sigma{ }^{b}\right) \in \Sigma$, $\sigma^{-1} \in \pi_{g}(L)$. Queremos $\sigma^{-1}(i) \in J$. Como $\mathrm{x} \in F i x(\Sigma)$, então $(l, \sigma) \mathrm{x}=\mathrm{x}$, isto $\left(l_{1} x_{\sigma^{-1}(1)}, \ldots, l_{N} x_{\sigma^{-1}\left(N^{\prime}\right)}\right)=\left(x_{1}, \ldots, x_{N}\right)$. Daí, $x_{i} \neq 0 \Rightarrow l_{i} x_{\sigma^{-1}(i)} \neq 0 \Rightarrow x_{\sigma^{-1}(i)} \neq 0 \Rightarrow$ $o^{-1}(i) \in J$. Portiato, $\pi_{g}(S) J \subset J$.

Agora mostranos (jue $\pi_{\mathcal{G}}(\Sigma)$ age transitivamente em $J$. Para isto, verificamos cule palla $a, j \in J\left(x_{i}, x_{j} \neq 0\right)$ existe $\sigma \in \pi_{\mathcal{G}}\left(\sum\right)$ till que $\sigma{ }^{\prime}(i)=j$. Suponhamos que näo existe

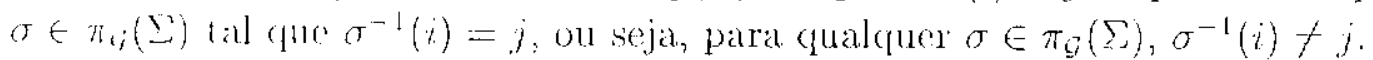

Sem perda de generalidade, suponhamos que não existe $\sigma \in \pi_{g}(\Sigma)$ tal que $\sigma^{-1}(1)=2$. Constrúmos princiranente o seguinte subconjunto de $J$,

$$
J_{1}=\left\{j \in J ; \sigma^{-1}(j) \neq 2, \forall \sigma \in \pi_{\mathcal{G}}(\Sigma)\right\} .
$$

Notemos que $1 \in J_{1}, \log 0, J_{1} \neq \emptyset$. Verificantos que $\pi_{\mathcal{G}}(\geq) J_{1}\left(J_{1}\right.$. De finto, nio existe $\delta \in \pi_{i}(2)$ lall que para $j \in J_{1}$

$$
\delta^{-1}(\underbrace{\sigma^{-1}(j)}_{\left(\pi_{\mathcal{G}}(\mathcal{2}) J_{1}\right.})=2,
$$

pois $\delta$ lo $^{1} \in \pi_{\mathcal{G}}(\Sigma)$ e ontäo $\delta^{-1} \sigma^{-1}(j) \neq 2$. Portanto, $\pi_{\mathcal{G}}(\Sigma) J_{1} \subset J_{1}$.

Agora, derinimos

$$
J_{2}=J_{j}^{c}=\left\{j \in J ; \sigma^{-1}(j)-2 \text {, palla algum } \sigma \in \pi_{\underline{L}}\left(\Sigma^{\prime}\right)\right\} .
$$

T'mos cue $2 \in J_{2}$ (considerande $\sigma$ a permulagào trivial); logo, $J_{2} \neq \varphi$. Verilicamos que $\pi_{G}(\mathrm{Y}) J_{2} \subset J_{2}$. Sejam $\sigma \in \pi_{G}(y)$ e $j \in J_{2}$. Queremos mostrar que $\sigma^{-1}(j) \in J_{2}$, isto é, que

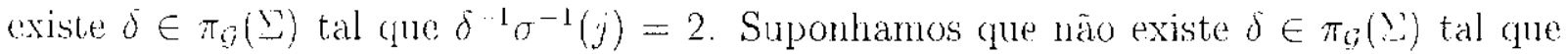

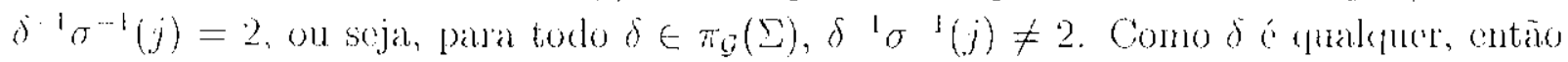
$\delta^{-1} \sigma^{-1}$ perene forlo $\pi_{g}(\Sigma)$ e então $j \in J_{1}$, o que e uma contradiça.

l'or construçáo, $J_{1}$ e $J_{2}$ sio subconjuntos disjuntos de $J$ Entrio, $\mathrm{y}=\left(y_{1}, \ldots, y_{N}\right)$ onclic

$$
y_{j}=\left\{\begin{array}{c}
x_{j} \text { so } j \in J_{1} \\
0 \text { se } j \notin J_{1}
\end{array}\right.
$$


$\therefore z=\left(z_{1}, \cdots, z_{N}\right)$ onde

$$
z_{j}=\left\{\begin{array}{l}
x_{j} \text { se } j \in J_{2} \\
0 \text { se } j \notin J_{2}
\end{array}\right.
$$

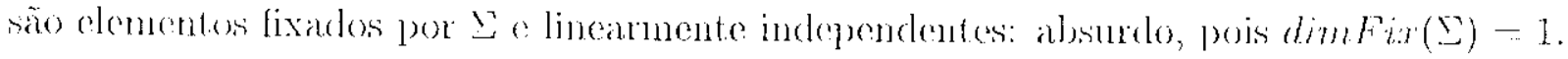
Portanto, $\pi_{g}\left(\begin{array}{l}3 \\ )\end{array}\right)$ age trasitivamente en $J$.

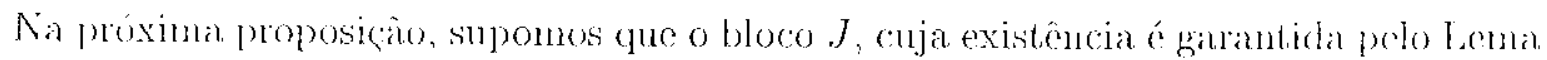
$2.3 .3,6 . J-\{1, \cdots, s\}$ onde $s<N$

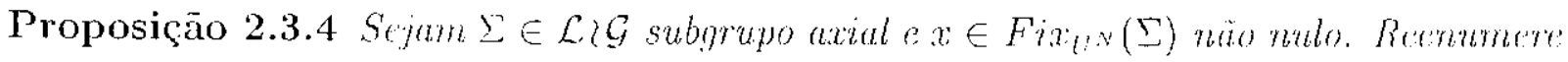

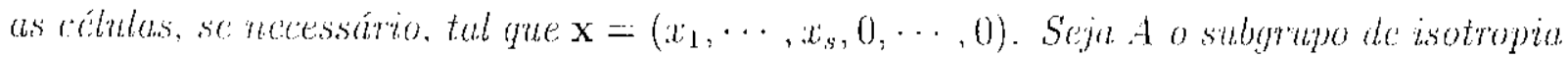
dex, an L. Lulain

a) I conjuryuto a $\Sigma(A, J)$

b) $1 \subset \mathcal{L}$ te axiul.

Demonstração Iniciamos provando que $\mathrm{x}$ o $\left(x_{1}, \cdots, x_{1}, 0, \cdots, 0\right)$ tem subgrupos

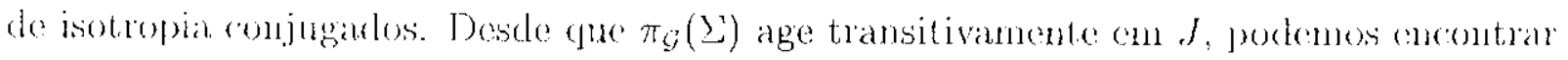
para cala $j \in f$ mon elemento $(l, o) \in \Sigma$ tal que $\sigma(1)=j$. Como $(l, \sigma) \mathbf{x}=\mathbf{x}$, lomos

$$
x_{j}=l_{j} x_{i r} \quad(j)=l_{j} x_{1}
$$

Soja $h-\left(l_{1}{ }^{1}, l_{2}, \ldots, l_{s}^{-1}, 1, \ldots, 1\right)$. Para $(k, \delta) \in \sum$ conclúnos com o Lema 1.1 .2 que

$$
\begin{aligned}
F r_{l N}\left(h \Sigma h^{-1}\right)-\quad & \mathbb{R}\left\{h\left(x_{1}, \ldots, x_{s}, 0, \ldots, 0\right)\right\} \\
& =\mathbb{R}\left\{\left(x_{1}, \ldots, x_{1}, 0, \ldots, 0\right)\right\} .
\end{aligned}
$$

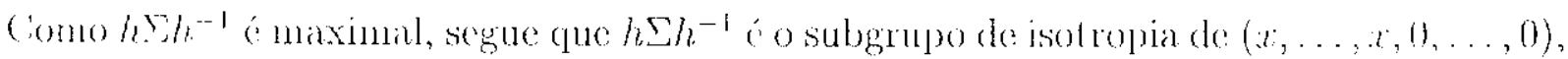
onde $x=x_{1}$ e como $\Sigma(A, J)$ fixa $(x, \ldots, x, 0, \ldots, 0)$, segue que $\Sigma(A, J) \subset h \Sigma h$. De maneina amáloga ao que foi feito na demonstraça do Lema 2.3.2 temos que $\Sigma(A, J)$, J $h \Sigma h^{-\cdots}$. Portanto, $\Sigma(h, J)=h \Sigma h^{-1}$.

Mostremos agota clue

$$
F x_{u N}(S(A, J))=\left\{\left(y_{1}, \ldots, y_{1}, 0, \ldots, 0\right) ; y_{1} \in F m_{u}(A)\right\}
$$

Seja y $-\left(y_{1}, \ldots, y_{s} ; y_{s+1}, \ldots, y_{N}\right) \in \operatorname{Pr} x_{U}\left(L^{2}(A, J)\right)$. Como y é lixado por $A^{s} \times \mathcal{L}^{N-s}$,

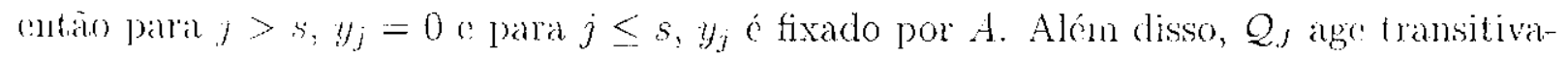

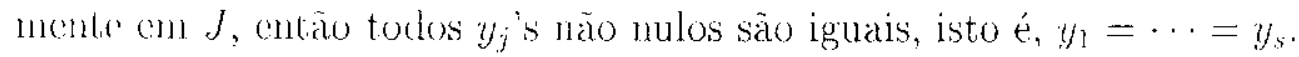

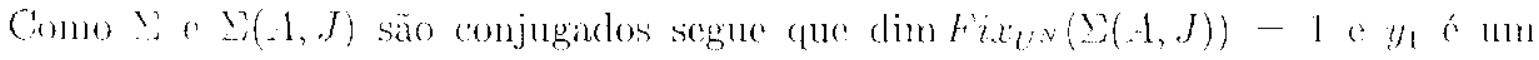

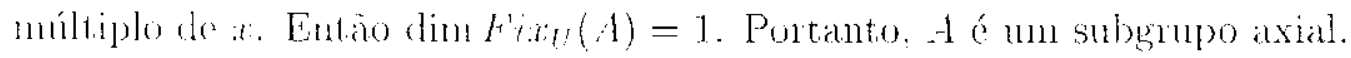




\subsubsection{Exemplos}

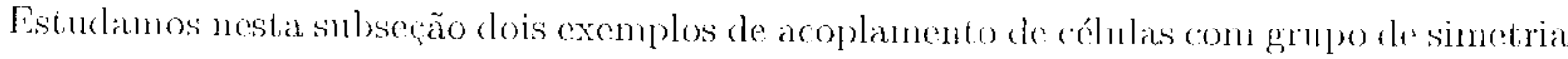

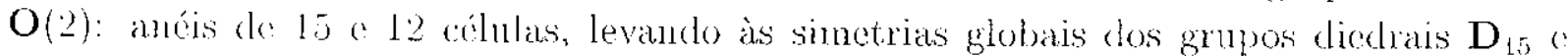

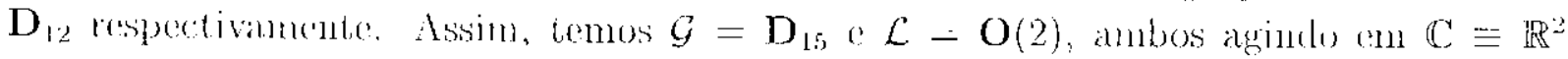

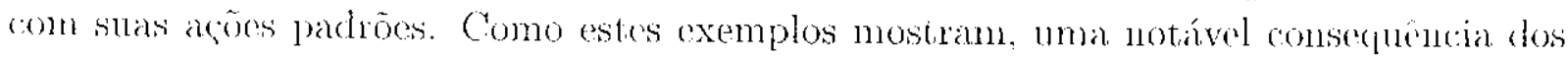
resultades gerais é que sistemas com acoplanento produto coroa frequentemente possinem

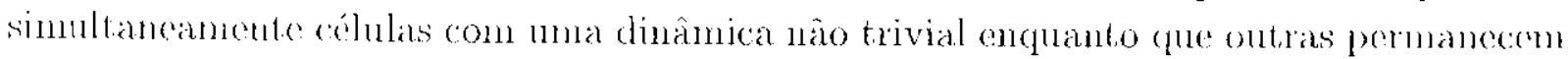
inations. Comecanos calculando os subgrupos axiais destes excmplos.

\section{a) Subgrupos axiais de $O(2), D_{15}$}

$S$ Soja $X=\{0,1,2, \cdots, 14\}$ en que $\mathrm{D}_{15}$ age por permutaçós cílicas e inversão. Iniciannos classificando os blocos $J$. Para isto, devemos concontran todos os subgrupos $\mathcal{H} \subset \mathcal{G}$, a menos de conjugacaio, e cntão os blucos são as $\mathcal{H}$-ombitas. Os possívois sulogrupos de $\mathbf{D}_{15}$ sào $\left.\mathbb{Z}_{3}=<R_{2 \pi / 3}\right\rangle, \mathbb{Z}_{5}=\left\langle R_{2 \pi / 5}\right\rangle, \mathbb{Z}_{15}=\left\langle R_{2 \pi / 15}\right\rangle$,

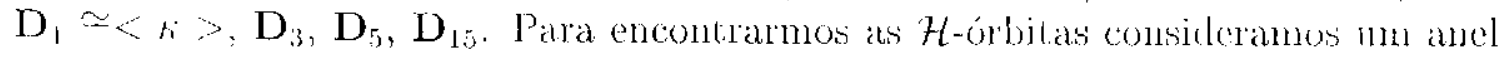
rircular com 15 cólulas e um eixo de simetria palssando polia cólula (). Notemos

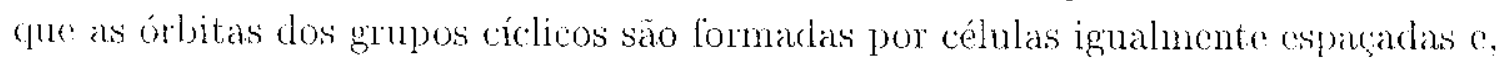

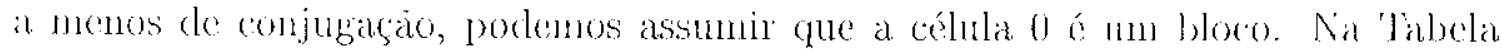
2.1 para os grupos diedrais somente listamos os blocos fue näo foram listados nos grupos cíclicos. l’or exemplo, as órbitas de $\mathrm{D}_{5}$ agindo $\mathrm{x}$. $\mathrm{X}$ sio $\{0,3,6,9,12\}$ e $\{1,2,1,5,7,8,10,11,13,14\}$, nats somente o segundo bloce é nove já (fue u primeiro ¿ lambrim una $\mathbb{Z}_{5}$-ólbita. Para cada bloco J já calculamos o subgrupo associalo $\mathcal{Q}_{j}=\left\{\sigma \in \mathbf{D}_{1 ;} ; \sigma(J)=J\right\}$, também mostrado na Tabela 2.1 .

\begin{tabular}{|c|c|c|}
\hline $\bar{G}$ & $J=$ células ativas & $Q_{J}$ \\
\hline 1 & $\{0\}$ & $\overline{\mathrm{D}_{1}}$ \\
\hline $\mathbb{Z}_{3}$ & $\{0,5,10\}$ & $\mathrm{D}_{3}$ \\
\hline $\mathbb{Z}_{5}$ & $\{0,3,6,9,12\}$ & $\mathrm{D}_{5}$ \\
\hline $\mathbb{Z}_{15}$ & $\{0, \ldots, 14\}$ & $\mathrm{D}_{15}$ \\
\hline $\mathrm{D}_{1}$ & $\{ \pm k\} ; h=1, \ldots, 7$ & $\mathrm{D}_{l}$ \\
\hline$D_{3}$ & $\{1,4,6,9,11,14\}$ & $\mathrm{D}_{3}$ \\
\hline $\mathrm{D}_{3}$ & $\{2,3,7,8,12,13\}$ & $\mathrm{D}_{3}$ \\
\hline $\mathrm{D}_{3}$ & $\{1,2,1,5,7,8,10,11,13,11\}$ & $\mathrm{D}_{1 ; ;}$ \\
\hline
\end{tabular}

'Tabela 2.1: Subgrupos axiatis de $\mathrm{O}(2)$ \& $\mathrm{D}_{15}$ a menos de comjugatgan

o) buice subgrupe de isotropia axial $A \subset \mathrm{O}(2)$ a menos de conjugagaion 6

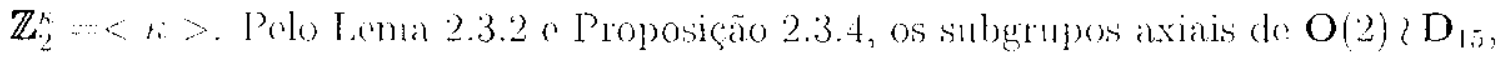

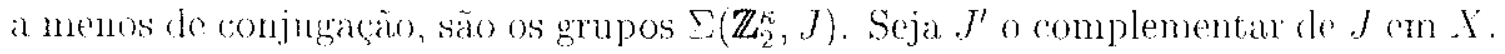

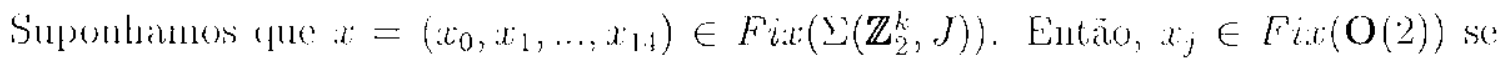
$j \in J^{\prime}$, isto $\in, x_{j}=0$ se $j \subset J^{\prime}$. Chamanos tais cólulas de inativas o todas as ontras 


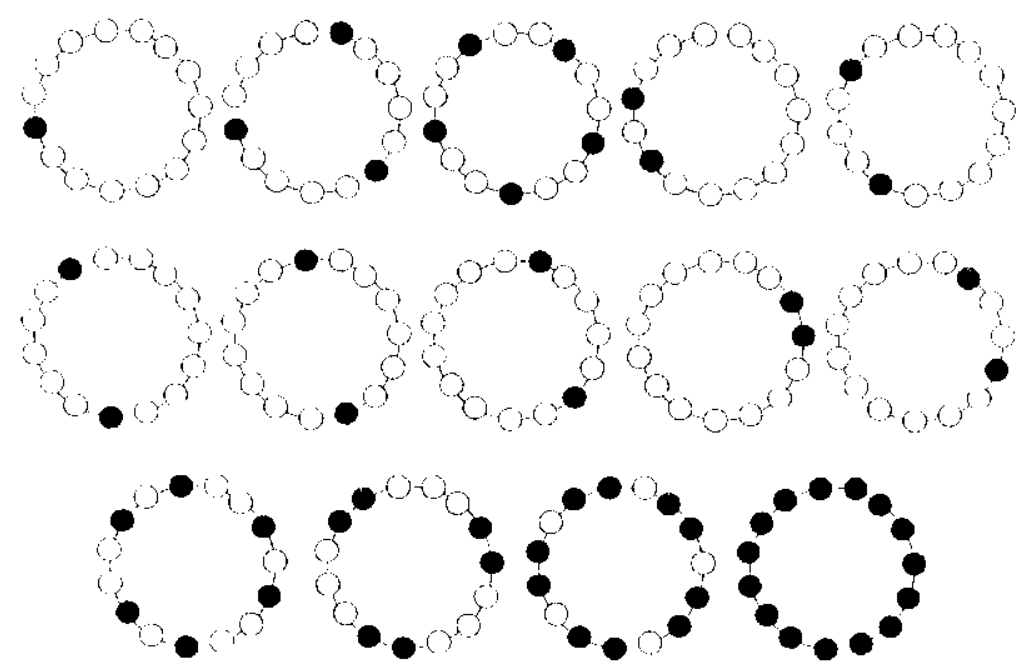

Figura 2.1: Diagramas cla células ativas/inativas en um anel de 15 cólulas idènticas com simetria inlestrat $\mathrm{O}(2)$.

(le ativas. Drsde que $\mathcal{Q}$, age transitivamente em $J$, então todas as cólulas ativas $x_{j}$ sino ignais para $j \in J$. Assim, qualquer posifa com subgrupo de isotropia $Z\left(\mathbb{Z}_{2}^{k}, J\right)$

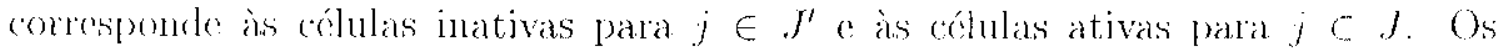
diagramas dat Figura 2.1 representam os 14 padroes de acoplamento das cóluas ati-

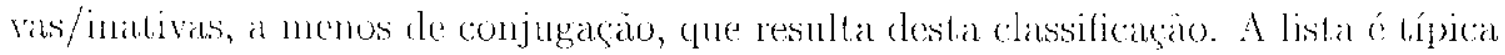

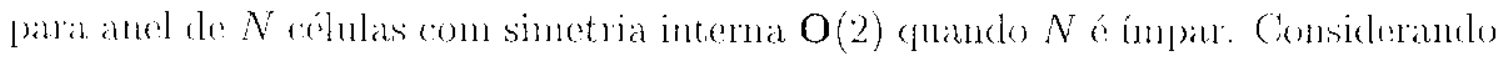
sine riats intemats mais complicadis somente impomos mais possiveis escolhas de A. A parte arucial ó a lista de blocos que depende somente de: $\mathcal{G}$.

\section{b) Subgrupos axiais de $\mathrm{O}(2) ; \mathrm{D}_{12}$}

Quanclo $N$ é palr a classificagrio ó similar, mas existem duas classes de conjugaçäo rlistintas de sulogrupos diedrais de mesma ordem.

Para $N=12$ considaramos $\xi=R_{\frac{2 \pi}{12}}$ o elemento de ordem 12 que gera $\mathbf{D}_{12}$ e $k 0$ alemente de orden 2. Sabemos que $h, \xi \hbar \in \mathbf{D}_{12}$ sato elementios de ordem 2 mas,

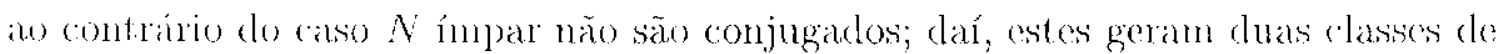
(onjugarao distintas.

Segundo o roteiro do exomplo anterior, calculanos primeinanente os subgrupes de

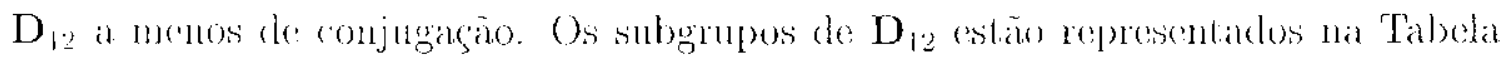
2.2 com as stas respertivas ordens.

Para o subgrupo de ordem 12 poderiamos cousiderar o subgrupe gerado pelos elemontes $\xi^{1}$ o $\xi^{3}$, ou seja, $\left\langle\xi^{4}, \xi^{3}\right\rangle$. Mas, para $j=0, \cdots, 110 l-0, \cdots, 11$,

$$
\frac{2 \pi}{+} j+\frac{2 \pi}{3} l=\frac{6 \pi j+8 \pi l}{12}=\frac{2 \pi}{12}(3 j+4 l)=\frac{2 \pi}{12} m
$$




\begin{tabular}{|c|c|}
\hline Ordem & Subgrupo \\
\hline 1 & trivial $=\left\{\mathrm{l}_{\mathrm{D}_{1}}\right\}$ \\
\hline 2 & $\mathbb{Z}_{2}=\left\langle\xi^{6}>\right.$ \\
\hline 2 & $\mathbb{Z}_{2}^{k}=<k:>$ \\
\hline 2 & $\mathbb{Z}_{2}^{\zeta \kappa}=\langle\xi \kappa\rangle$ \\
\hline 3 & $\mathbb{Z}_{3}=\left\langle\xi^{1}\right\rangle$ \\
\hline 4 & $\mathbf{D}_{2}=\left\langle\xi^{6}, k\right\rangle$ \\
\hline 4 & $\mathrm{D}_{2}^{\xi \kappa}=\left\langle\xi^{(i}, \xi \kappa>\right.$ \\
\hline 6 & $\left.\mathbf{D}_{3}=<\xi^{4}, f_{i}\right\rangle$ \\
\hline 6 & $\mathbf{D}_{3}^{\xi / i}=\left\langle\xi^{1}, \xi \kappa>\right.$ \\
\hline 8 & $\mathbf{D}_{1}=\left\langle\xi^{3}, k\right\rangle$ \\
\hline 8 & $\mathbf{D}_{1}^{\xi k}=\left\langle\xi^{3}, \xi, k>\right.$ \\
\hline 12 & $\mathbb{Z}_{12}$ \\
\hline 12 & $\mathbf{D}_{6}=\left\langle\xi^{2}, k\right\rangle$ \\
\hline 12 & $\mathbf{D}_{6}^{\xi i r}=\left\langle\xi^{2}, \xi_{k}>\right.$ \\
\hline 21 & $\mathbf{D}_{12}=\langle\xi, k\rangle$ \\
\hline
\end{tabular}

Tabela 2.2: Subgrupos de $\mathbf{D}_{12}$

$m=0,1,2, \ldots, 11$, isto $i$, os elementos deste subgrupo sấ as rotaçós de $\frac{2 \pi}{12} m$, para $m=0,1,2, \ldots, 11$, que é conjugado a $\mathbb{Z}_{12}$.

A partir dos subgrupos obtemos os blocos e então os subgrupos $\mathcal{Q}$, a presentados na. 'labela 2.3. Na Figura 2.2 temos os 15 diagramas partröes de acoplamento das células ativas/inativas cune resultam da Tabela 2.3 .

Observação 2.3.5 Nos cxemplos a) e b) notamos a ocorrêneia simultânea de cólulas ativas e inativats. Fste fenoneno ó consequência das regras de acoplanente de produto coroa, o que - sob certas circunstancias - poderia de fato clesacoplar cólulas inativas de suls virinhancas. Mais geralnente, assumimos que Fix $(\mathcal{L})=\{0\}$ e tomannos un subcomjunto qualuner $K^{\prime} \subset\{1,2, \cdots, N\}$ năo necessariamente um bloco. Consideramos unu subconjunto $\Upsilon \subset \mathcal{L}\} \mathcal{G}$ da loma

$$
\Upsilon=B_{1} \times \cdots \times B_{N}
$$

onde

$$
B_{k}=\left\{\begin{array}{cc}
\mathcal{L} & \text { se } k \in K \\
1 & \text { caso contrário. }
\end{array}\right.
$$

Entiño

$$
F i x \Upsilon=F i x\left(B_{1}\right) \times \cdots \times F i x\left(B_{N}\right)
$$

oncte:

$$
\text { Fir }\left(B_{k}\right)=\left\{\begin{array}{cc}
0 & \text { se } k \in K \\
U & \text { calso contrário }
\end{array}\right.
$$




\begin{tabular}{|c|c|c|}
\hline $\mathcal{G}$ & $J=$ células ativas & $\mathcal{Q}_{j}$ \\
\hline 1 & $\{0\}$ & $\mathbb{Z}_{2}$ \\
\hline $\mathbb{Z}_{2}$ & $\{0,6\}$ & $\mathrm{D}_{2}$ \\
\hline $\mathbb{Z}_{2}^{n}$ & $\{ \pm \mathrm{j}\} ; \mathrm{j}-1, \ldots, 6$ & $\mathrm{D}_{2}$ \\
\hline $\mathbb{Z}_{2}^{\xi \kappa}$ & $\{0,1\},\{2,11\},\{3,10\}$ & $\mathrm{D}_{2}^{\zeta \kappa}$ \\
\hline $\mathbb{Z}_{3}$ & $\{0,1,8\}$ & $\mathrm{D}_{3}$ \\
\hline $\mathbb{Z}_{1}$ & $\{0,3,6,9\}$ & $D_{4}$ \\
\hline $\mathrm{D}_{2}$ & $\{1,5,7,11\}$ & $\mathrm{D}_{2}$ \\
\hline $\mathrm{D}_{2}^{\xi k}$ & $\{0,1,6,7\}$ & $\mathrm{D}_{?}^{\xi \kappa}$ \\
\hline $\mathbb{Z}_{6}$ & $\{0,2,4,6,8,10\}$ & $\mathrm{D}_{6}$ \\
\hline $\mathrm{D}_{3}$ & mesinos blocos do $\mathbb{Z}_{3}$ e: $\mathbb{Z}_{6}$ & $\mathbf{D}_{3}$ \\
\hline$D_{3}^{\xi \kappa i}$ & $\{0,1,4,5,8,9\}$ & $\mathbf{D}_{3}^{\xi \kappa}$ \\
\hline $\mathrm{D}_{1}$ & $\{1,2,4,5,7,8,10,11\}$ & $\mathrm{D}_{1}$ \\
\hline $\mathbf{D}_{i}^{\xi_{i}}$ & $\{1,2,1,5,7,8,10,11\}$ & $\mathbf{D}_{4}^{\xi \pi i}$ \\
\hline $\mathbf{D}_{6}$ & $\{1,3,5,7,9,11\} \equiv\{0,2,4,6,8,10\}$ & $\mathrm{D}_{\mathrm{fi}}$ \\
\hline $\mathrm{D}_{6}^{\mathrm{fi}_{i}}$ & $\mathrm{X}$ & $\mathrm{D}_{\xi}^{\xi_{k}}$ \\
\hline $\mathbb{Z}_{12}$ & $X$ & $\mathbf{D}_{12}$ \\
\hline $\mathbf{D}_{12}$ & $X$ & $\mathrm{D}_{12}$ \\
\hline
\end{tabular}

Tabela 2.3: Subgrupos axiais do $\mathrm{O}(2)$ ? $\mathrm{D}_{12}$

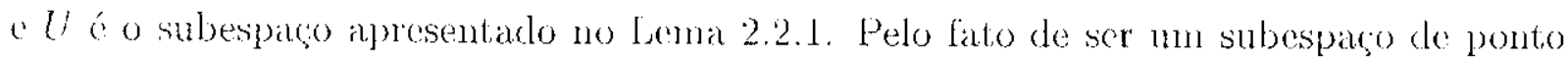
fixo, tal subsspaço é invariante pela dinânica. Qualquer solução nào nula da restrição (la LDO original au Fra $\Upsilon$ é uma posição do sistema em que as cóhulas en $E^{\circ}$ são todas inativas. Além clisso, as cólulas ativas não são necessarianente idênticas. Assimin, seria possivel, por exemplo, manjaj as células onde o comportamento de algumas delas fosse caúticu encluanto clue células na sua vizinhança permanecessem inativas.

\subsubsection{Subgrupos de isotropia e subgrupos de isotropia maximais de $\mathcal{L}$ iG}

$S$ ciann $\Sigma_{1}, \ldots, \Gamma_{s}$ subgrupos de isotropia de $\mathcal{L}$ não conjugados dois a dois. Soja

$$
J=J_{1} \cup \cdots \cup J_{r}
$$

uma partiçio do conjunto $\{1,2, \cdots, N\}$, tal que $r \leq s$. Um subeonjunto $J_{i}$ e channato parte da particio J. Seja

$$
\mathcal{Q}_{\jmath}=\left\{\sigma \in \mathcal{G}: \sigma\left(J_{i}\right)-J_{i}, \text { para } 1 \leq i \leq \eta\right\} .
$$

Para simplilicar us índices definimos

$$
\mathcal{X}:\{1, \ldots, N\} \rightarrow\{1, \ldots, r\}
$$

por

$$
X(i)=k \text { para } i \in J_{k}
$$




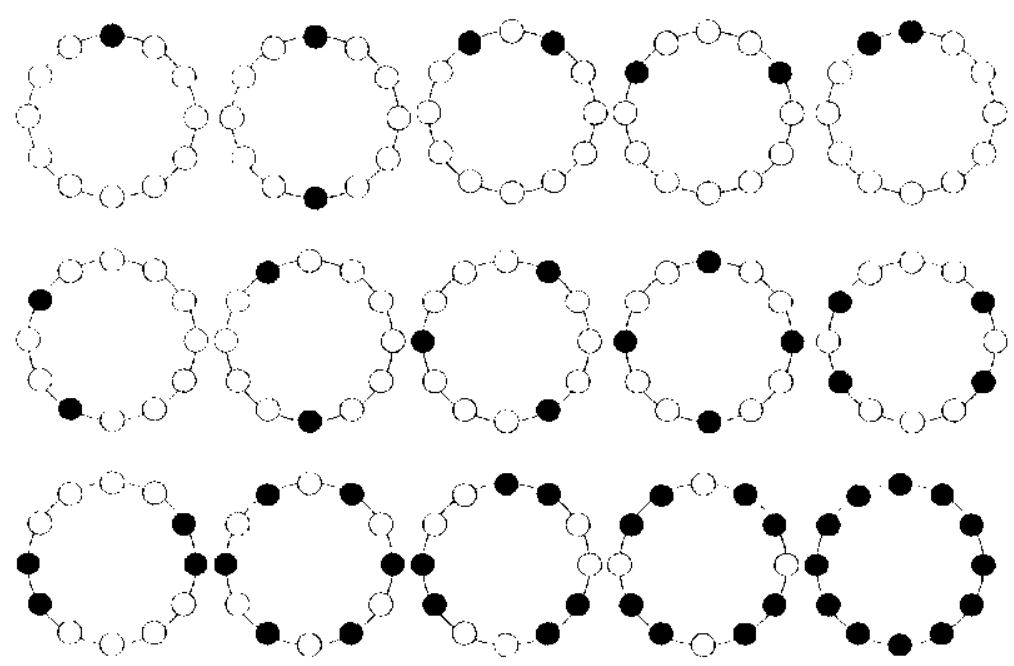

Figura 2.2: Jiagranas da cólulas ativas/inativas em un anel de 12 células idénticas con simetria ittloma $\mathrm{O}(2)$.

Assim, $\mathcal{X}(i)$ denota a parte de $J$ a que $i$ pertence. Denotamos $\Sigma_{J}=B_{1} \times \cdots \times B_{N}$, onde $B_{i}=\Sigma_{x(i)}$. Finalnente, definimos

$$
\Sigma=\Sigma_{J}+\mathcal{Q}_{J}
$$

A proposicaio a seguir mostra que $\sum$ em (2.3.6) é um subgrupo cle isotropia. Fica claro na denomstrangu que a desigualdade $r \leq s$ e a condiça de $\Sigma_{1}, \ldots, \Sigma_{s}$ sercm subgrupos do isotropia näo conjugados dois a dois são anıbas hipóteses necessárias para. $\Gamma$ ser um subgrupo de isotropia.

Proposição 2.3 .6 I como em (2.3.6) é um subgrupo de isotropia de $\Gamma=\mathcal{L} ३ \mathcal{G}$ aggindo em $U^{N}$ e lodo subgrupo de isotropia de $\Gamma$ é conjugado a um tal $\Sigma$.

Demonstração $\quad$ Seja $w_{i} \in U$ um vetor cujo subgrupo de isotropia em $\mathcal{L}$ é $\Sigma_{i}$, para $i=1, \ldots, s$. Soja $v=\left(v_{1}, \ldots, v_{N}\right)$ onde $v_{i}=w_{x(i)}$. Por construciá, $\Sigma$ lixa $v$. Deste que

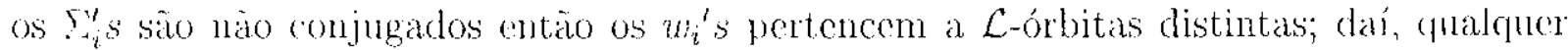
elonento de $\mathcal{L}\{\mathcal{G}$ que fixa $v$ deve preservar as partes de $J$. Segue que nonhum elemento

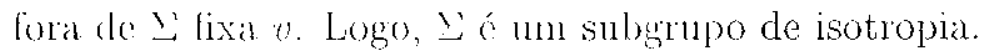

Reciprocamente, consideranos o subgrupo de isotropia $\Sigma$ do $v=\left(v_{1}, \cdots, v_{N}\right) \in U^{N}$.

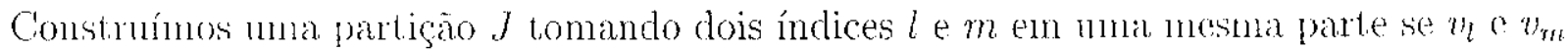
pertencen a una mesmá órbita de $\mathcal{L}$. Consideranos $f=J_{1} \cup \cdots \cup J_{r}$ a particáo acima

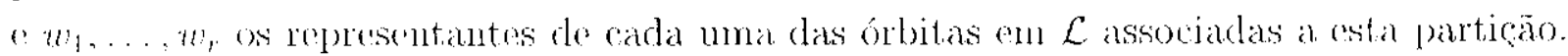
Daí, se $j \in J_{v}$ então $v_{j}=l_{\imath} w_{i}$ para algum $l_{i} \in \mathcal{L}$. Logo, $v=l$ w onde $l \in \mathcal{L}^{N}$ a d $j$-ésima

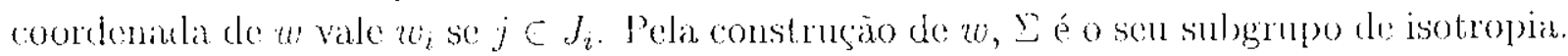
Se considemanos o $\in Q_{j}$ temess que $v-(l, \sigma) w$, on seja, $v$ e $w$ pertencem a mma mesmai $\mathcal{L}$ (G-órbita. Logo, o subgrupo de isotropia de $v$ é conjugarlo ao subgrupo do isotropia $\Sigma$. 
A partir do subgrupo de isotropia 2 podemos calcular seu subespaco de ponto fixo. Refinamos a partiģăo f a uma partigào $K$ onde o subgrupo Qd age transitivantente em adia parte de $h$. Definanos $\rho(i)=j$ se $i$ pertence a $j$ ćsima parte de $K$. Entio

$$
F x_{j N}(\Omega)-\left\{\left(z_{1}, \ldots, z_{N}\right) E U^{N} ; z_{i}=z_{j} \text { se } \rho(i)-\rho(j)\right\},
$$

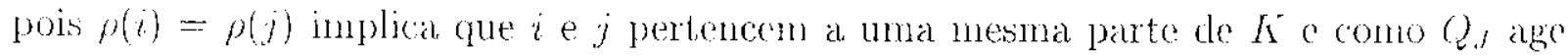
tramsitivamente com carla parte de $K$, então $z_{i}=z_{j}$.

Agera consideramos $J_{i}^{K}$ o número de partes de $K$ que estão contidats na parte $J_{i}$ da partiçäe . J. Jintão

$$
\operatorname{din} F x_{U^{N}}\left(y_{i}\right)=\sum_{i=1}^{s} J_{i}^{K}\left(\lim F x_{U} \sum_{i}\right.
$$

jai que $\operatorname{Frr}\left(B_{1} \times \cdots \times B_{N}\right)-\operatorname{Fix} B_{1} \times \cdots \times \operatorname{Fix} B_{N}$.

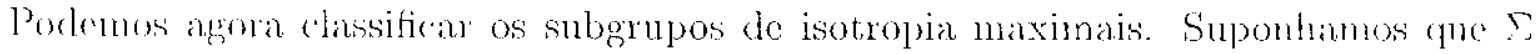
6 mom subgrupo de isotropia maximal correspondente a una partiçăo J. Como $\Sigma$ pode ber annentado trocando un dos $\Sigma_{j}$ 's por $\mathcal{L}$, então un destes $\Sigma_{j}$ 's deve ser o próprio $\mathcal{L}$, diganos $\Sigma_{2}$ (correspondente à parte $J_{2}$ ). Além disso, como $\Gamma$ e moximal, isto fanbém implica que existe somente mais una parte $J_{1}$ correspondente a um subgrupo do isotropia

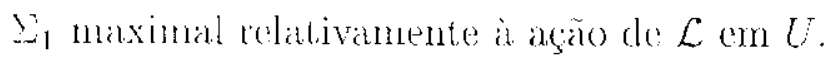

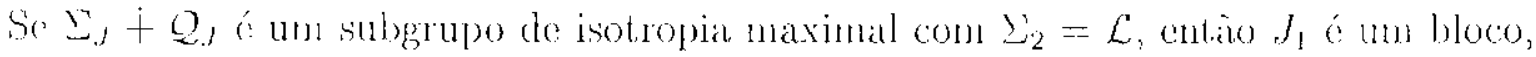

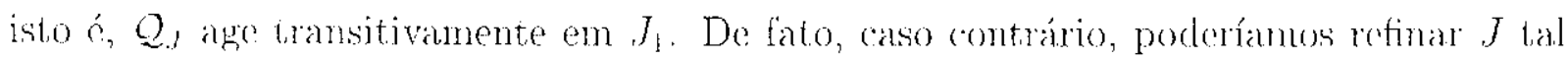
que: Q.j agisse transitivanente nas novas partes de $J_{1}$. Então, novanente, poderíanos ammentare o subgrmo de isotropia tomando $\Sigma_{1}=\mathcal{L}$ em todas as partes de $J_{1}$, exceto om

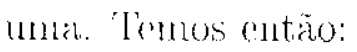

Proposiçāo 2.3.7 Torbo subyrupo de isotropia maximal en $\mathcal{L} \backslash \mathcal{G}$ á da fomm $\Sigma=\Sigma_{J}+\mathcal{Q}_{J}$, onde J é uma particalo de $\{1,2, \ldots, N\}$ com no mámimo duas partes, digamos, $J=J_{1} \cup J_{2}$,

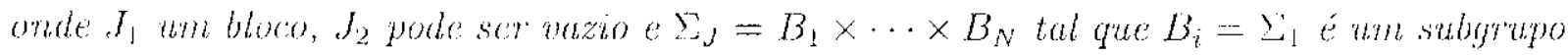
de dsolnopha matmal, se $i \in J_{1} \& B_{i}=\mathcal{L}$ si $i \in J_{2}$.

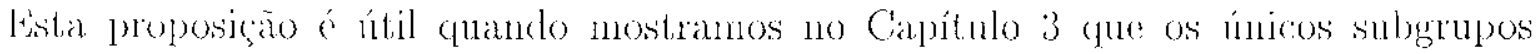
de isotropia de $\mathrm{O}(2)$ i $\mathrm{S}_{N}$ (ple geram rames de solugoes de um problema de bifurcateno $\mathrm{O}(2)<\mathrm{S}_{N-\mathrm{e} q u i v a t i a n t e}$ sáo os subgrupos axiais. 


\section{Capítulo 3}

\section{Bifurcação de pontos de equilíbrio com simetria $\mathbf{O}(2) \imath \mathbf{S}_{N}$}

Nosso objetivo neste capitulo é usar a teoria apresentada no Capítulo 2 para encontrar us parluós em bifurcaça de pontos de equilíbrio para sistents de $N$ células acopladas (iclenticanonte) cluas a duas, onde cada célula tem sinceria inlerma $O(2)$. A frigura 3.1 ilustra acoplamentos de 4 ou jónélulas duas a duas. O grupo produto coroa em questão é $\mathrm{O}(2) / \mathrm{S}_{N}$. Suponos que a dinâmica de carda célula ocorre no plano o entáa consideramos a aça padraio de $\mathrm{O}(2)$ em $\mathbb{C}$ (ver Exemplo 1.1.5-4).

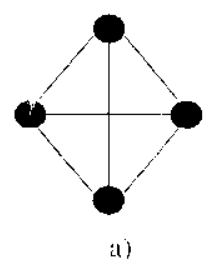

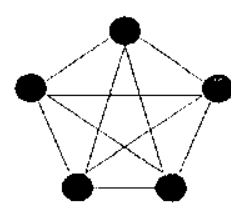

b)

Figura 3.1: a) Acoptauncuto de 4 células duas a duas: $\mathcal{G}=\mathbf{S}_{4}$, b) Acoplamento de 5 cólulas dults a duats: $9=\mathrm{S}$,

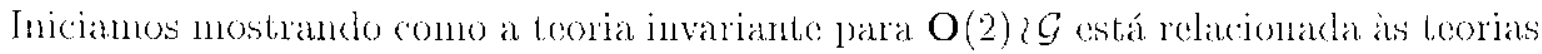
de: $\mathrm{O}(2)$ o de $\mathcal{G}$, onde $\mathcal{G}$ ó un subgrupo de $\mathrm{S}_{N}$. Depois, aplicanos estes resultados ao grupo $\mathrm{O}(2)<\mathrm{S}_{N}$.

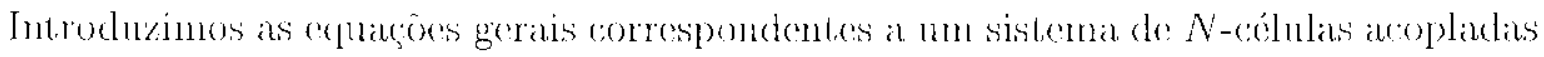
duas a duas. A partir das soluços correspondentes aos subgrupos de simetria com subespaços de ponto lixo unidimensional encontranos o padtão genérico de soluçoes de chuilíbrio. Verilicanos que, a menos de conjugaçio, existem somente $N$ tipos de subgrupos de simetria com subespax̧os de ponto lixo unidinensional. Cienericamente, o Lema des liamos Equivariantes garante a existencia de solugoes con estas simetrias. Para deter-

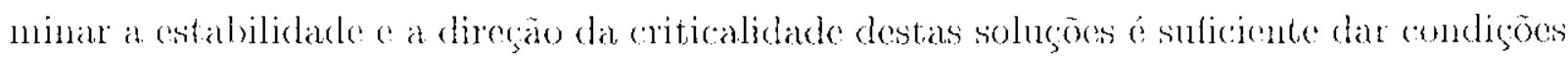
para os cocticiontes dos teruos de ordem menor do que tres na equaçào gemal do caupo de vetores. Além disso, os autovalores asseriados às derivadas calcularlas em ada una

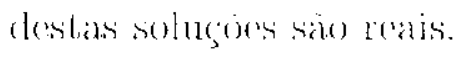


Finalmente, nostranos que genericamente os únicos ramos de solucoes para problemas de bifurcaça com simetria $\mathrm{O}(2)$ \& $\mathrm{S}_{N}$ são aquneles obtidos pelo Lema clos Ramos Equivarianters.

\subsection{Teoria invariante e equivariante para o grupo $\mathrm{O}(2)<\mathcal{G}$}

Consictere o grupo $\mathrm{O}(2)$ com a aça do Exemplo 1.1.5-1, $\mathcal{G}$ um subgrupo de $\mathrm{S}_{N}$ e IV um espaço vetorial de dimensão finita. Consideramos a ação de $\mathcal{G}$ em $W^{N}$ daxla por (1.1.9).

() teorema a seguir mostra como podemos clescrever a cstrutura do and $\mathrm{P}(\mathrm{O}(2)<\mathcal{G})$ a partir do $P(O(2))$ e $\mathrm{P}(\mathcal{G})$.

Teorema 3.1.1 Saja $Y^{-}-\mathbb{C}$. Seja $\mathcal{G}$ um subgrupo de $\mathrm{S}_{N}$ e $\Gamma=\mathrm{O}(2)<\mathcal{G}$. Considerumos a acruo de $\Gamma$ en $Y^{N}$ como em (1.1.11) e a ação de $\mathcal{G}$ em $\mathbb{R}^{N}$ como em (1.1.9).

$S e \mu_{1}, \ldots \mu_{s}$ fonman um base de Hilbert para $\mathcal{P}(\mathcal{G})$, entäo para bodo $f \in \mathcal{E}(\Gamma)$ existe $h \in \varepsilon_{\text {s }}$ lal $q u t$

$$
f(z) \cdots h\left(\mu_{1}\left(z_{1} \bar{z}_{1}, \ldots, z_{N} \bar{z}_{N}\right), \ldots, \mu_{s}\left(z_{1} \bar{z}_{1}, \ldots, z_{N} \bar{z}_{N}\right)\right)
$$

para $\approx=(\infty, \ldots, \approx) \in V^{N}$

P’ara demonstrar aste teorema ntilizamos a invariancia sob $\mathrm{O}(2)^{N^{N}}$ :

Lema 3.1.2 Seja $V=\mathbb{C} e p: V^{N} \rightarrow \mathbb{R}$ uma funçáo polinomial. Se pé invariante sob

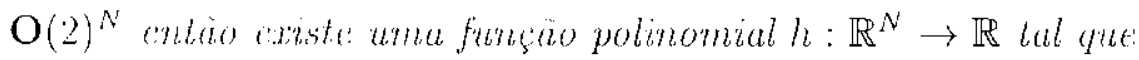

$$
p(z)=h\left(z_{1} \bar{z}_{1}, \ldots, z_{N} \bar{z}_{N}\right)
$$

para $z=\left(2 !, \ldots, z_{N}\right) \subset V^{N}$

Demonstraçāo $\quad \operatorname{Soja} p: \mathbb{C}^{N} \rightarrow \mathbb{R}$ uma função polinomial invariante sob $\mathrm{O}(2)^{N}$. Podenos ciscrever $p$ hats coordenaklas $z_{1}, \bar{z}_{1}, \ldots, z_{N}, \bar{z}_{N}$ ent $V^{N}$ na formal

$$
p(z)=\sum_{\iota, ; j} a_{\kappa \beta \beta} z^{\left(\alpha \bar{z}^{\beta}\right.}
$$

onde $a_{n \beta} \in \mathbb{C} \cdot\left(r, \beta \in\left(\mathbb{Z}^{1}\right)^{N}\right.$.

Comop ó uma função real entrão $p=\bar{p}$, ou seja

$$
\sum_{\alpha, \beta} a_{\alpha \beta \beta} z^{\alpha} \bar{z}^{\beta}=\sum_{\alpha, \beta} \bar{h}_{\alpha, \beta} \bar{z}_{z}^{\alpha} z^{\beta} .
$$

$\log \left(0, a_{i a_{2}}=\pi_{t r i \gamma}\right.$.

Como p ( $\mathrm{O}(2)^{N}$-invarialute, entäo

$$
p\left(e^{i \theta_{1}} z_{1}, \ldots, e^{i \theta_{N} z_{N}}\right)=p\left(z_{1}, \ldots, z_{N}\right) .
$$


para todo $\theta=\left(\theta_{1}, \ldots, \theta_{N}\right) \in \mathrm{S}^{N}$, ou seja,

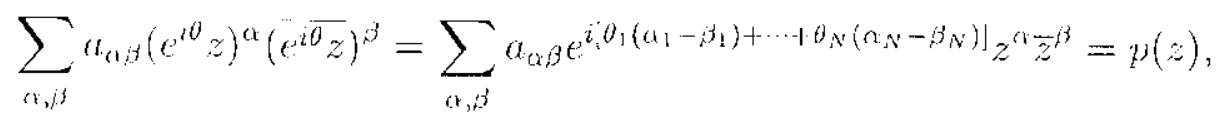

omle $z=\left(z_{1}, \ldots, z_{N}\right), z^{0}-\left(z_{1}^{\alpha_{1}}, \ldots, z_{N}^{\left(\alpha_{N}\right)}\right) 0 z^{0-z^{\beta}}=z_{1}^{a_{1}} z_{1}^{\beta_{1}} \ldots z_{N}^{r_{N}} \bar{z}_{N}^{\beta_{N}}$.

Em (3.1.1) tomos (1ue

$$
a_{a, \beta}=a_{\alpha \beta} e^{i\left[\theta_{1}\left(\alpha_{1}-\beta_{1}\right)+\cdots+\theta_{N}\left(\alpha_{N}-\beta_{N}\right)\right]} .
$$

Daí,

$$
\begin{gathered}
a_{x \beta \beta}=0 \text { ou } \\
\alpha_{1}=\beta_{1} \text { e. } \alpha_{N}=\beta_{N} .
\end{gathered}
$$

Portinito,

$$
p(z)=\sum a_{k \alpha}\left(z_{1} \bar{z}_{1}\right)^{\alpha_{1}} \ldots\left(z_{N} z_{N}\right)^{a_{N}}
$$

onde $a_{\text {ctax }} \in \mathbb{R}$.

Se tomambos $h: \mathbb{R}^{N} \rightarrow \mathbb{R}$, tal que $h(x)=\sum a_{c x c} x_{1}^{\alpha_{1}} \ldots x_{N}^{\alpha_{N}}$, temos o resultado desejado.

\section{Demonstração (Teorena 3.1.1)}

Como $\mathcal{G}$ ó um subgrupo do $\mathrm{S}_{N}$, segue do Teorema 1.3.5 a existência de mua base de Hilbert finita $\left\{\mu_{1}, \ldots, \mu_{s}\right\}$ pira o anel $\mathcal{P}(\mathcal{G})$. Agora pelo resultado do Schwar\% (Teorema 1.3.8) é suticionte nostrar (que $\mu_{1}\left(z_{1} \bar{z}_{1}, \ldots, z_{N} \bar{z}_{N}\right), \ldots, \mu_{s}\left(z_{1} \bar{z}_{1}, \ldots, z_{N} \bar{z}_{N}\right)$ geraun o intel $P(\Gamma)$

Soja $p: I^{N} \rightarrow \mathbb{R}$ tal que $p \in \mathcal{f}\left(\mathrm{l}^{2}\right)$. Em particular, $p$ é $\mathrm{O}(2)^{N}$-invariante e polo lema 3.1 .2 temos que existe $h: I^{N} \rightarrow \mathbb{R}$ tal que

$$
p(z)=h\left(z_{1} \bar{z}_{1}, \ldots, z_{N} \bar{z}_{N}\right)
$$

Além dissso, $p$ é $\mathcal{G}$-invariante o que implica $h \mathcal{G}$-invariante, vu seja, existe $\ddot{h} \in \mathcal{E}_{s}$ lal (jue.

$$
h\left(x_{1}, \ldots, x_{N}\right)=\tilde{h}\left(\mu_{1}\left(x_{1}, \ldots, x_{N}\right), \ldots, \mu_{s}\left(x_{1}, \ldots, x_{N}\right)\right) .
$$

Conclúmos que $\mu_{1}\left(z_{1} \bar{z}_{1}, \ldots, i_{N} \bar{z}_{N}\right), \ldots, \mu_{s}\left(z_{1} \bar{z}_{1}, \ldots, z_{N} \bar{z}_{N}\right)$ é uma base do ITilbert do $\mathcal{P}(\mathrm{\Gamma})$. Segue do Teorema 1.3 .8 que se $f \in \mathcal{E}(\Gamma)$ então existe $g \in \mathcal{E}_{s}$ tal que

$$
f(z)=g\left(\mu_{1}\left(z_{1} \bar{z}_{1}, \ldots, z_{N} \bar{z}_{N}\right), \ldots, \mu_{s}\left(z_{1} \bar{z}_{1}, \ldots, z_{N} \bar{z}_{N}\right)\right)
$$

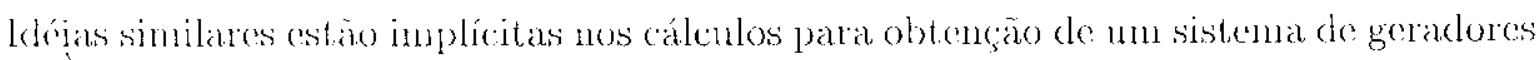
pain $\vec{E}(\mathrm{O}(2)(\mathcal{G})$ : 
Teorema 3.1.3 Seja $V=\mathbb{C}$ um subgrupo do $\mathrm{S}_{N} \in \Gamma=\mathrm{O}(2)<\mathcal{G}$. Considere a açör de I' em $\mathbb{C}^{N}$ a a acio de $\mathcal{G}$ no $\mathbb{R}^{N}$ como definida anteriormente.

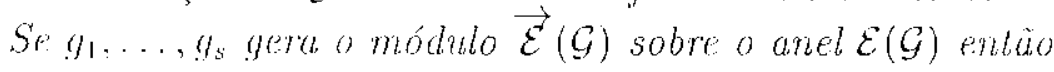

$$
g_{1}\left(z_{1} \bar{z}_{1}, \ldots, z_{N} \bar{z}_{N}\right) * z_{1} \ldots, g_{s}\left(z_{1} \bar{z}_{1}, \ldots, z_{N} z_{N}\right) * z
$$

ycro a módulo $\overrightarrow{\mathcal{C}}(\Gamma)$ sobre o anel $\mathcal{E}(\Gamma)$, onde $z=\left(z_{1}, \ldots, z_{N}\right) \subset V^{N}$ a o simbolo $*$ entre dois vetores ésado para denotar o vetor obtido multiplicando componentes de mesmo indice desses vetores.

Para proxar o Teorema 3.1 .3 usamos a equivariância sob $\mathrm{O}(2)^{N}$ a partir do lema a seguir.

Lema 3.1.4 Sija $V-\mathbb{C}$ eq: $V^{N} \rightarrow V^{N}$ uma aplicacáo com componentes polmomiais.

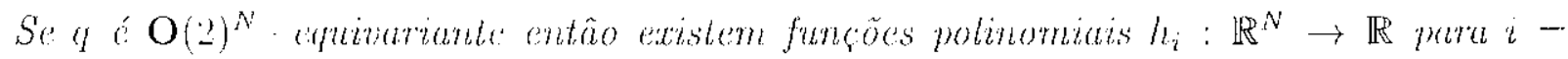
$1, \ldots, N$ tais que

$$
\eta(z)=\left(h_{1}\left(z_{1} \bar{z}_{1}, \ldots, z_{N} \bar{z}_{N}\right) z_{1}, \ldots, h_{N}\left(z_{1} \bar{z}_{1}, \ldots, z_{N} z_{N}\right) z_{N}\right),
$$

para lodo $z=\left(z_{1}, \ldots, \Sigma_{N}\right) \in V^{N}$.

Demonstração Suponhamos $q \in \vec{P}\left(\mathrm{O}(2)^{N}\right)$, onde q $=\left(q_{1}, \ldots, q_{N}\right)$ com $\eta_{i}: V^{N} \rightarrow V$ para $i=1, \ldots, N$. Entáa, para todo $\theta-\left(\theta_{1}, \ldots, \theta_{N}\right) \in \mathrm{S}^{\mathbf{N}^{N}}, z \in V^{N}$ temos

$$
\theta q(z)=q\left(e^{t \theta_{1}} z_{1}, \ldots, e^{2 \theta_{N}} z_{N}\right)
$$

l'or ontro bialos.

$$
\theta q(z)=\left(e^{i \theta_{1}} q_{1}(z), \ldots, e^{i \theta_{N}} q_{N}(z)\right)
$$

Assim, pala $j=1, \ldots, N$,

$$
q_{j}(z)=e^{-i \theta_{j}} q_{j}\left(e^{i \theta_{1}} z_{1}, \ldots, e^{i \theta_{N}} z_{N}\right) .
$$

Nas coordenatiats $z_{1}, \bar{z}_{1}, \ldots, z_{N}, \bar{z}_{N}$ escrevemos

$$
q_{1}(z)=\sum_{\alpha, \beta} a_{\alpha \beta \beta} z^{<k} \bar{z}^{\beta}
$$

Constrteratulo $j=1 \mathrm{~cm}(3.1 .2)$ temos

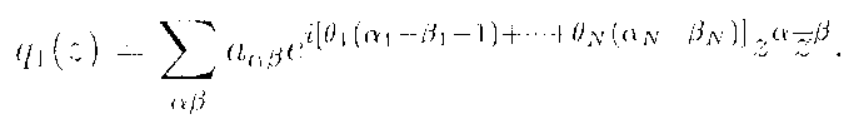

$1)(3.1 .3) \times(3.1 .4)$, segue que

$$
\begin{gathered}
a_{\alpha^{\prime} \beta}-0 \text { ou } \\
\alpha_{1}=\beta_{1}+1 \text { e. } \alpha_{N}=\beta_{N} .
\end{gathered}
$$


Assinill,

$$
q_{1}(i)=\sum a_{\beta_{1}+1} \beta_{1} \beta_{2} \beta_{2} \beta_{N} \beta_{N}\left(z_{1} \bar{z}_{1}\right)^{\beta_{1}} \ldots\left(z_{N} z_{N}\right)^{\beta_{N}} z_{1} .
$$

Seja

$$
h_{1}(x)=\sum \operatorname{Re}\left(a_{\beta_{1}+1 \beta_{1} \beta_{2} \beta_{2} \ldots \beta_{N} \beta_{N}}\right) x^{\beta^{3}}
$$

(

$$
t_{1}(x)=\sum \operatorname{Im}\left(a_{B_{1}+1 \beta_{1}, \beta_{3} \beta_{2} \ldots \beta_{N} \beta_{N}}\right) x^{\beta}
$$

Fition,

$$
q_{1}(z)-h_{1}\left(z_{1} \bar{z}_{1}, \ldots, z_{N} \bar{z}_{N}\right) z_{1}+i l_{1}\left(z_{1} \bar{z}_{1}, \ldots, z_{N} \bar{z}_{N}\right) z_{1} .
$$

Nós tambrum temos

$$
q\left(k_{i} z_{1}, \ldots, z_{N}\right)=\left(k q_{1}(z), q_{2}(z), \ldots, q_{N}(z)\right)
$$

que inplical que $q_{1}\left(z_{1}, z_{2}, \ldots, z_{N}\right)-\overline{q_{1}(z)}$. Portanto,

$$
t_{1}\left(\sim \sim_{\bar{z}}, \ldots, z_{N} \bar{\sim}_{N}\right) \equiv 0
$$

De mancita análoga, mostramos que para $i=2, \ldots, N$ existem outros $h_{i}$ tais que?

$$
q_{i}(z)=h_{i}\left(z_{1} \bar{z}_{1}, \ldots, z_{N} \ddot{z}_{N}\right) z_{i} .
$$

Portinto.

$$
q(z)=\left(h_{1}\left(z_{1} \bar{z}_{1}, \ldots, z_{N} \bar{z}_{N}\right) z_{1}, \ldots, h_{N}\left(z_{1} \bar{z}_{1}, \ldots, z_{N} z_{N}\right) z_{N}\right) .
$$

\section{Demonstração (Teorena 3.1.3)}

Descle gue $\mathcal{G}$ a um grupo de Lie compacto agindo no $\mathbb{R}^{N}$, temos pelo Teorema 1.3 .18 (ane existe: un múmero finito de polinômios que geram o módulo $\overrightarrow{\mathcal{P}}(\mathcal{G})$, digamos, $g_{1}, \ldots, g_{2}$.

Mustromos (que

$$
\left\{g_{1}\left(z_{1}, z_{1}, \ldots, z_{N} \bar{z}_{N}\right) * z_{2} \ldots, g_{s}\left(z_{1} \bar{z}_{1}, \ldots, z_{N} \bar{z}_{N}\right) * z\right\}
$$

gera o módulo $\vec{P}(\Gamma)$.

Polo Lema 3.1.1, se $q \in \overrightarrow{\mathcal{P}}\left(\mathrm{O}(2)^{N}\right)$, entäo existem funçöes polinomiais $h_{i}: \mathbb{R}^{N} \rightarrow \mathbb{R}$ parat $i=1, \ldots, N$ tais que?

$q(z)=\left(h_{1}\left(z_{1} z_{1}, \ldots, z_{N} z_{N}\right), \ldots, h_{N}\left(z_{1} z_{1}, \ldots, z_{N} z_{N}\right)\right) * z=\left(h_{1}, \ldots, h_{N}\right)\left(z_{1} \bar{z}_{1}, \ldots, z_{N} \bar{z}_{N}\right) * z$.

Além disso, se $\eta \in \overrightarrow{\mathcal{P}}(\mathcal{G})$, então $h=\left(h_{1}, \ldots, h_{N}\right) \in \overrightarrow{\mathcal{P}}(\mathcal{G})$ c

$$
h\left(x_{1}, \ldots, x_{N}\right)-\sum_{i=1}^{r} p_{i}\left(x_{1}, \ldots, x_{N^{N}}\right) g_{i}\left(x_{1}, \ldots, x_{N}\right)
$$


oncle $p_{1}: \mathbb{R}^{N} \rightarrow \mathbb{R}$ saio polincimios $\mathcal{G}$-invariantes. I'ortanto,

$$
q(z)=\sum_{i=i}^{r} p_{i}\left(z_{1} \bar{\Sigma}_{1}, \ldots, z_{N} \bar{z}_{N}\right) g_{i}\left(z_{1} \bar{z}_{1}, \ldots, z_{N} \bar{z}_{N}\right) * z,
$$

ondre conclúnos que a conjunto $\left\{g_{1}\left(z_{1} \bar{z}_{1}, \ldots, z_{N} \bar{z}_{N}\right) * z, \ldots, g_{s}\left(z_{1} \bar{z}_{1}, \ldots, z_{N} \bar{z}_{N}\right)+z\right\}$ ó bease do módulo $P(\Gamma)$ sobre o anel $P(\Gamma)$.

Segur do Toorema 1.3.19 que este conjunto gera o módulo $\vec{E}(\Gamma)$ sobre o and $\mathcal{E}(\Gamma)$.

\subsection{Teoria invariante e equivariante para o grupo $\mathbf{O}(2)<\mathrm{S}_{N}$}

A partir dos resultades da segão anterior obtemos a forma geral para $u m$ problema de bifuracgio (on simetria $\mathrm{O}(2)$ i $\mathrm{S}_{\mathrm{N}}$. Mas antes, vamos encontrar a forma geral de $u$ m geme $f \in \vec{E}\left(S_{N}\right)$. Para isto precisamos do Teorema fundamental das limçoes simétricas, (jule veremos a seguir.

Definição 3.2.1 Seja $R$ un anel comutativo arbitrário com elentento adendidade. Um

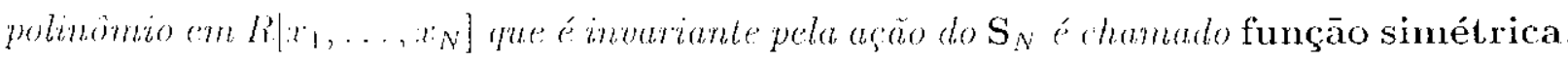

Veremos ane sito exomples de luncoes simétricas somas o produtos polinomios dar formal $s_{p}=\sum_{i}^{N} x_{i}^{\prime},($ m) $110 p \in \mathbb{N}$.

Introdurindo mua nova indeterminada $z$ e considerando a lumegro

$$
f(z)=\left(z-x_{1}\right)\left(z-\cdots x_{2}\right) \ldots\left(z-x_{N}\right)
$$

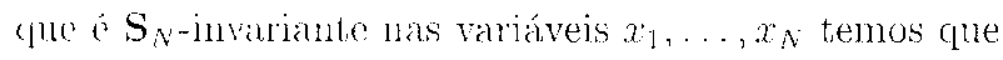

$$
f(z)=\left(z-x_{1}\right)\left(z-x_{2}\right) \ldots\left(z-x_{N}\right)=z^{N}-\sigma_{1} z^{N-1}+\sigma_{2} z^{N \cdots 2}+\ldots(-1)^{N} \sigma_{N},
$$

onde os coeficientes sä́o dades por

$$
\begin{aligned}
& \sigma_{1}=x_{1}+\cdots+x_{N} \\
& \sigma_{2}=x_{1} x_{2}+x_{1} x_{3}+\cdots+x_{2} x_{3}+\cdots+x_{N-1} x_{N} \\
& \sigma_{3}-x_{1} x_{2} x_{3}+x_{1} x_{2} x_{1}+\cdots+x_{N-2} x_{N-1} x_{N} \\
& \vdots \\
& \sigma_{N}=x_{1} x_{2} \ldots x_{N} .
\end{aligned}
$$

O coeticiente $\sigma_{i}$ é a somatória de todos os possiveis produtos com orden i nas indeterui.

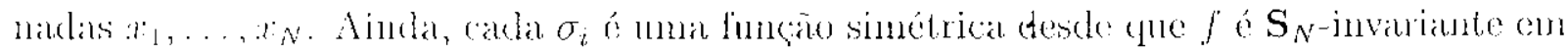
$x_{1}, \ldots, x_{N}$. Chandanos $\sigma_{1}, \ldots, \sigma_{N}$ funções simétricas elementares cm $x_{1}, \ldots, x_{N}$. 
Un pulinomio da format $\varphi\left(\sigma_{1}, \ldots, \sigma_{N}\right)$ á una funçio sinuétrica de $x_{1}, \ldots, x_{N}$. Lm

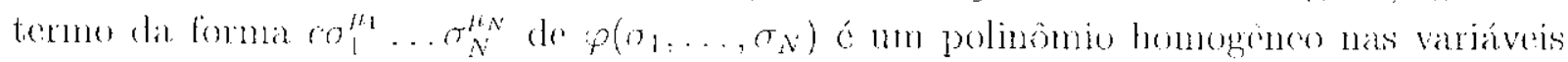
$x_{1}, \ldots, x_{N}$ de grau $\mu_{1}+2 \mu_{2}+\cdots+N \mu_{N}$, descle que carla $\sigma_{i}$ o mom polinomio homogenoo de ordem i. A somal $\mu_{1}+2 \mu_{2}+\cdots+N \mu_{N}$ é chamada de peso do termo e $\sigma_{1}^{h_{1}} \ldots \sigma_{N}^{h_{N}}$. o

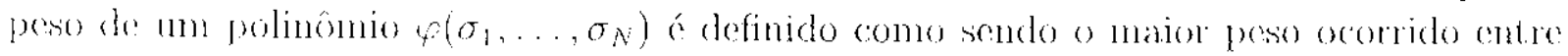
$\cos$ tomoses $\sigma_{1}^{\prime \prime} \ldots \sigma_{N}^{n N}$.

Teorema 3.2.2 (Tromema fundamental das funsós simétricas) Un polinônio simétrico de grau ho wo antel polinomial $R\left[x_{1}, \ldots, x_{N}\right.$ pode ser escrito como $\varphi\left(\sigma_{1}, \ldots, o_{N}\right)$ de peso hi, onter $\sigma_{1}, \ldots, \sigma_{N}$.

Observação $3.2 .3 \mathrm{Su}$ em anbos os membros de $(3.2 .5)$ tomanos $x_{N}=0$, tomus

$$
\left.\left(z-a_{1}\right) \ldots\left(z-a_{N-1}\right) z=z^{N}-\left(\sigma_{1}\right)_{0} z^{N 1}+\cdots+(-1)^{N-1}\left(\sigma_{N}\right)_{0}\right)_{0}
$$

onde $\left(\sigma_{i}\right)_{0}$ a a cxpressio que obtemos tomando $x_{N}=0$ em $\sigma_{i}$. So dividimos a expressão acima por is obtomos

$$
\left(z \quad x_{1}\right) \cdots\left(z-x_{N-1}\right)=z^{N-1}-\left(\sigma_{1}\right)_{0} z^{N \cdots 2}+\cdots+(-1)^{N-1}\left(\sigma_{N} 1\right)_{0}
$$

Vesta expaciäo temos que $\left(\sigma_{1}\right)_{0}, \ldots,\left(\sigma_{N-1}\right)_{0}$ são os coeficientes da funço simćtrica de orden $N-1$ nats indeterminadas $x_{1}, \ldots, x_{N-1}$.

Demonstração (Teorena fundamental das funçoes simétricas)

A prova segue por indusa em $N$. Para $N=1$ o teorema cerdadeiro, todo polinônio

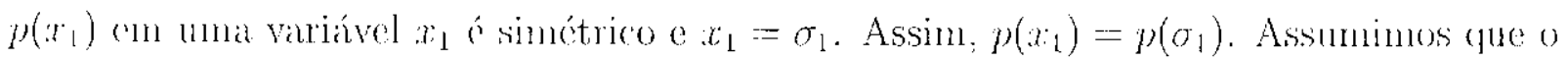
teorena vale para todo polinômio nas $N-1$ variáveis $x_{1}, \ldots, x_{N-1}$. Vamos verificar que o teoremal vale paral polinônios nas $N$ variáveis $x_{1}, \ldots, x_{N-1}, x_{N}$.

Para polimomios de grau zero niss $N$ variáveis o teorema é trivial, pois o polinomió constante. Então assumimos que o teorema está provado para todo polinomio de gráu $<k$.

Seja $p\left(n_{1}, \ldots, x_{N}\right)$ un polinônio simétrico de gran h. Tomando $x_{N}-0$, temos pou hipótese de indugiono que

$$
p\left(x_{1}, \ldots, x_{N-1}, 0\right)=\varphi\left(\left(\sigma_{1}\right)_{0}, \ldots,\left(\sigma_{N}, 1\right)_{0}\right)
$$

onde $\varphi$ está om lunçio das funçöes elencutares sinétricas nas indeterminadas $x_{1}, \ldots, x_{N-1}$ é de peso $\leq h$. Portianto, $\varphi\left(\sigma_{1}, \ldots, \sigma_{N-1}\right)$ tem peso $\leq k$.

Comstruínos

$$
\mu_{1}-\mu\left(x_{1}, \ldots, x_{N-1}, x_{N}\right)-\varphi\left(\sigma_{1}, \ldots, \sigma_{N-1}\right)
$$

() polinomio $p_{1}\left(x_{1}, \ldots, x_{N}\right)$ é simétrico. O primeiro termo do lado dircito é de grau $k$, o segundo tem peso $\leq k$ e assim como um polinômio nas variáveis $\left(x_{1}, \ldots, x_{N}\right)$ tem gran $\leq h$, conseguentemente $p_{1}$ é de grau $\leq k$. Além disso, para $x_{N}=0, p_{1}$ é nulo; logo, todos 
os termos possuen a variável $x_{N}$. Desde que a função $p_{1}$ é simótrica, todos os termos

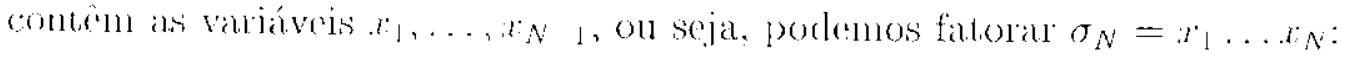

$$
\mu_{1}=\sigma_{N}\left(x_{1}, \ldots, x_{N}\right)
$$

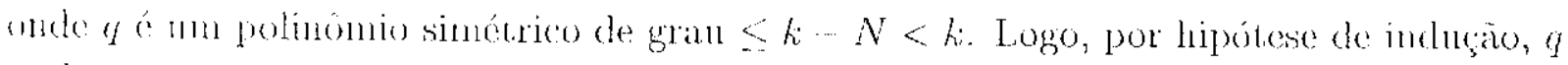
pode ser escrita om funça de $\sigma_{1}, \ldots, \sigma_{N}$ :

$$
q=w\left(\sigma_{1}, \ldots, \sigma_{N}\right)
$$

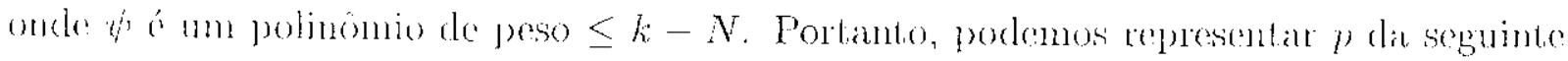
Cormin:

$$
p=\mu_{1}+\varphi\left(\sigma_{1}, \ldots, \sigma_{N-1}\right)=\sigma_{N} \psi\left(\sigma_{1}, \ldots, \sigma_{N}\right)+\varphi\left(\sigma_{1}, \ldots, \sigma_{N^{\prime}-1}\right) .
$$

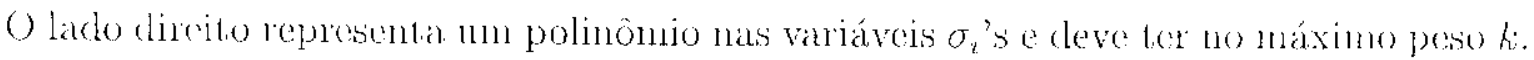

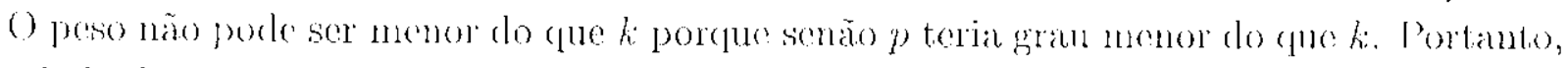
o lato cliroito possuj peso exatamente $k$.

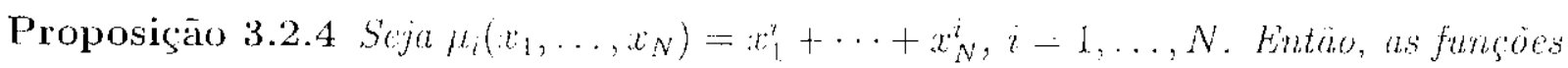

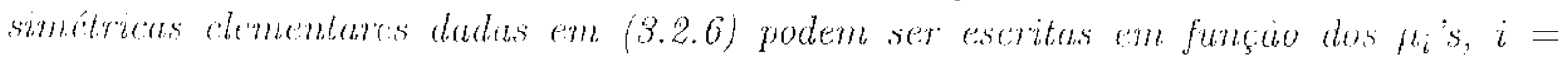
$1, \ldots, N$

Demonstração Consileramos $c_{i}=(\cdots 1)^{i} \sigma_{i}$, tal quo $\prod_{i=1}^{N}\left(1-x_{i} l\right)=\sum_{i=0}^{N} c_{i} l^{i}$.

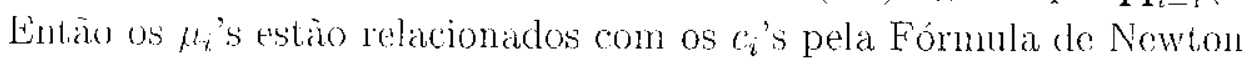

1. $\mu_{r}+c_{1} \mu_{r-1}+c_{2} \mu_{r \cdot 2}+\cdots+c_{r-1} \mu_{1}+c_{r} r=0$ se $1 \leq r \leq N$

2. $\mu_{r}+c_{1} \mu_{r-1}+c_{2} \mu_{r-2}+\cdots+c_{N} \mu_{r \cdots N}=0$ sor $r \geq N$

Como $r<N$ matáo considermos a equação 1 . Tomando $r=1, c_{1}=-\mu_{1}$. Parar $r=2$,

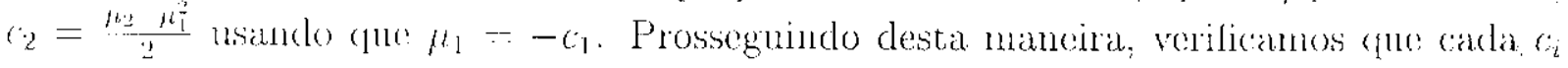

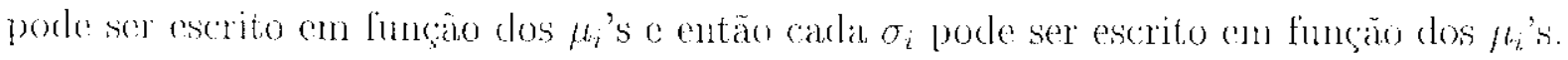

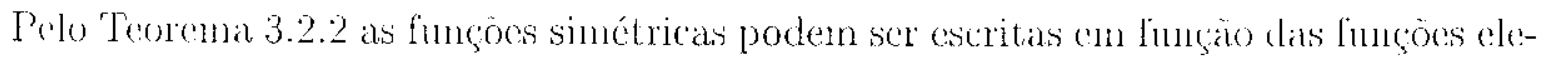

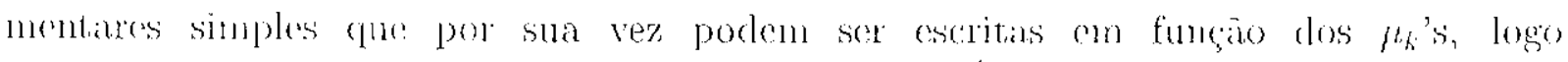
$\left\{h_{k} ; k=1, \ldots, N\right\}$ ó uma base de Hilbert de $\mathcal{P}\left(\mathrm{S}_{N}\right)$. É com estab base (1ue trabalhamos até u fintal deste capitulo.

Corolário 3.2.5 Seja $f: \mathbb{C}^{N} \rightarrow \mathbb{R}$ um germe suave. $S c f \in \mathcal{P}\left(\mathrm{O}(2), \mathrm{S}_{N}\right)$, entüo existe $h \in \varepsilon_{N}$ lal qub

$$
f(z)=h\left(\mu_{1}, \ldots, \mu_{N}\right)
$$

$\operatorname{para} z=\left(x_{1}, \ldots, z N\right) \in \mathbb{C}^{N}$

$$
\mu_{k}=\left(z_{1} \bar{z}_{1}\right)^{k}+\cdots+\left(\tilde{\sim}_{N} \ddot{z}_{N}\right)^{k}
$$

para $h:=1, \ldots, N$. 
Demonstração $\quad P^{3}$ cho Teorema 3.2 .2 e Proposiça $3.2 .1\left\{\mu_{k} ; k-1, \ldots, N\right\}$ é una hatse de Ililluert de: $\mathcal{P}\left(\mathbf{S}_{N}\right)$, o resultado segue do Teorema 3.1.1.

Antos de apmesentarmos o próximo corolário, vanos encontran a base do módulo $\vec{E}\left(S_{N}\right)$ solore o ated $\mathcal{E}\left(\mathrm{S}_{N}\right)$.

Lema 3.2 .6 omódulo $\overrightarrow{\mathcal{E}}\left(\mathrm{S}_{N}\right)$ r gerado sobre o cuncl $\mathcal{E}\left(\mathrm{S}_{N}\right)$ por

$$
\left(\begin{array}{c}
3 x_{1}^{k} \\
x_{2}^{k} \\
\vdots \\
x_{N}^{k}
\end{array}\right)
$$

parak $k=0, \ldots, N-1$.

Demonstração Pelo leorema 1.3 .19 restringimos nossa atenção aos polinomios.

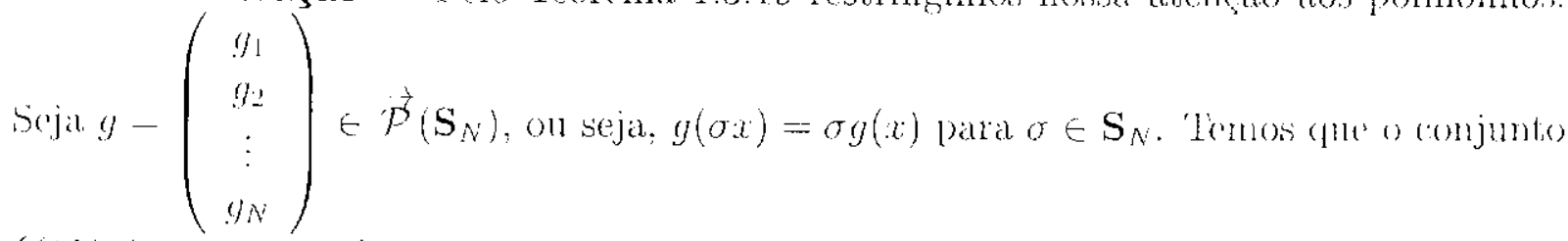

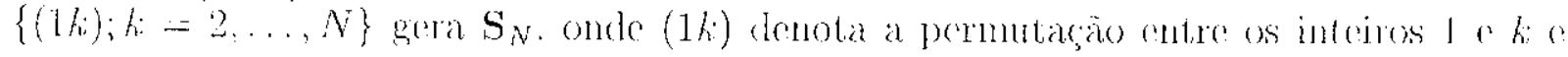
lixa os demalis $N-2$ inteinos de $1, \ldots, N$.

$$
\begin{gathered}
\text { Para } \sigma=\left(1 f_{i}\right), x=\left(x_{1}, \ldots, x_{N}\right) \text { e } y=\left(\begin{array}{c}
g_{1} \\
g_{2} \\
\vdots \\
g_{N}
\end{array}\right) \text {, tomos } \\
\sigma y(x)=\sigma\left(\begin{array}{c}
g_{1}(x) \\
\vdots \\
g_{k}(x) \\
\vdots \\
g_{N}(x)
\end{array}\right)=\left(\begin{array}{c}
g_{k}(x) \\
\vdots \\
g_{1}(x) \\
\vdots \\
g_{N}(x)
\end{array}\right) .
\end{gathered}
$$

Por ontro lario,

$$
\left(\begin{array}{c}
g_{1}(\sigma x) \\
\vdots \\
g_{k}(\sigma x) \\
\vdots \\
g_{N}(\sigma x)
\end{array}\right)=\left(\begin{array}{c}
g_{1}\left(x_{k}, \ldots, x_{1}, \ldots, x_{N}\right) \\
\vdots \\
g_{k}\left(x_{k}, \ldots, x_{1}, \ldots, x_{N}\right) \\
\vdots \\
g_{N}\left(x_{k}, \ldots, x_{1}, \ldots, x_{N}\right)
\end{array}\right)
$$

Darí,

$$
y_{k}\left(x_{1}, \ldots, x_{k}, \ldots, x_{N}\right)=y_{1}\left(x_{k}, \ldots, x_{1}, \ldots, x_{N}\right)
$$




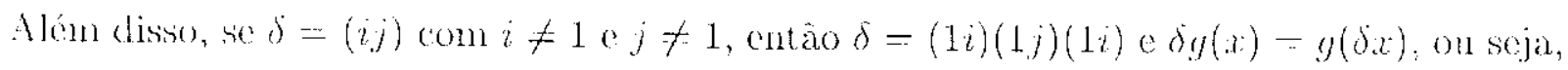

$$
\left(\begin{array}{c}
y_{1}\left(x_{1}, \ldots, x_{i}, \ldots, x_{j}, \ldots, x_{N}\right) \\
\vdots \\
g_{j}\left(x_{1}, \ldots, x_{1}, \ldots, x_{j}, \ldots, x_{N}\right) \\
\vdots \\
g_{i}\left(x_{1}, \ldots, x_{2}, \ldots, x_{j}, \ldots, x_{N}\right) \\
\vdots \\
g_{N}\left(x_{1}, \ldots, x_{i}, \ldots, x_{j}, \ldots, x_{N}\right)
\end{array}\right)=\left(\begin{array}{c}
g_{1}\left(x_{1}, \ldots, x_{j}, \ldots, x_{i}, \ldots, x_{N}\right) \\
\vdots \\
g_{1}\left(x_{1}, \ldots, x_{j}, \ldots, x_{i}, \ldots, x_{N}\right) \\
\vdots \\
g_{j}\left(x_{1}, \ldots, x_{j}, \ldots, x_{1}, \ldots, x_{N}\right) \\
\vdots \\
g_{N}\left(x_{1}, \ldots, x_{j}, \ldots, x_{1}, \ldots, x_{N}\right)
\end{array}\right) .
$$

$\log 0, g_{1}\left(x_{1}, \ldots, x_{1}, \ldots, x_{1}, \ldots, x_{N}\right)=g_{1}\left(x_{1}, \ldots, x_{j}, \ldots, x_{i}, \ldots, x_{N}\right)$.

Jortinto,

$$
g(x)=\left(\begin{array}{c}
y_{1}\left(x_{1}, \ldots, x_{N}\right) \\
g_{1}\left(x_{2}, x_{1}, x_{3}, \ldots, x_{N}\right) \\
\vdots \\
g_{1}\left(x_{N}, x_{2}, \ldots, x_{N-1}, x_{1}\right)
\end{array}\right)
$$

onclo

$$
g_{1}\left(x_{1}, \ldots, x_{i}, \ldots, x_{j}, \ldots, x_{N^{\prime}}\right)=g_{1}\left(x_{1}, x_{2}, \ldots, x_{j}, \ldots, x_{i}, \ldots, x_{N^{N}}\right)
$$

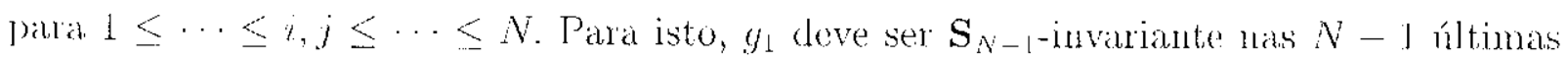

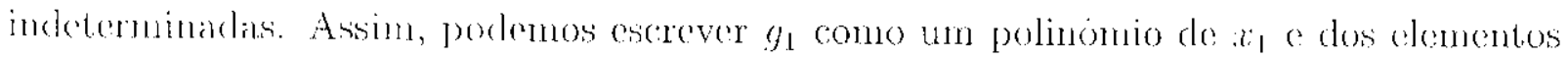
da base de flibure de $\mathcal{P}\left(\mathrm{S}_{N-1}\right)$, on sejar:

$$
\left.g(x)=y_{1} x_{1} x_{2}+x_{3}+\cdots+x_{N}, x_{2}^{2}+x_{3}^{2}+\cdots+x_{N}^{2}, \ldots, x_{2}^{N-1}+x_{3}^{N-1}+\cdots+x_{N}^{N}{ }^{1}\right),
$$

Snsim.

$$
\begin{aligned}
& g(x)=
\end{aligned}
$$

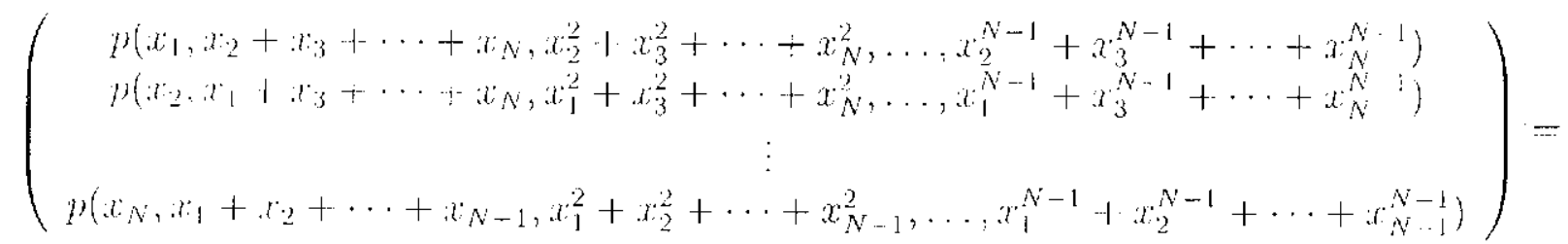

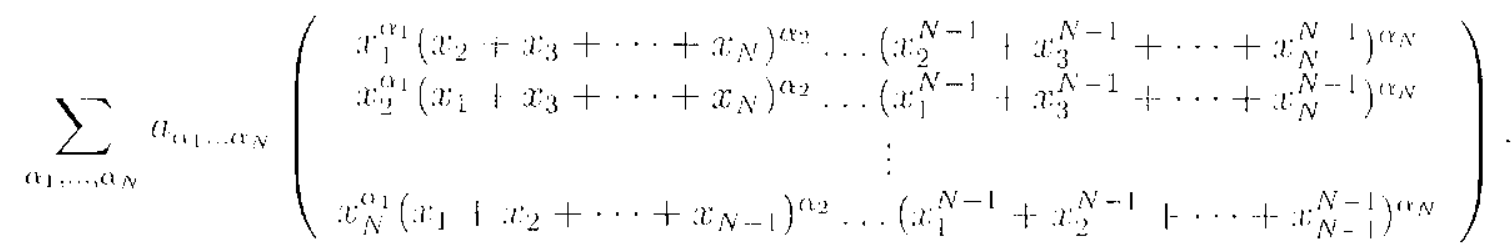

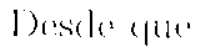

$\left(X_{1}+X_{2}+\cdots+X_{N}\right)^{n}-\left(X_{2}+\cdots+X_{N}\right)^{\alpha}=\left[X_{1}+\left(X_{2}+\cdots+X_{N}\right)\right]^{t+}-\left(X_{2}+\cdots+X_{N}\right)^{\alpha}=$

$$
X_{1}^{u}+\sum_{i=1}^{n-1}\left(\begin{array}{c}
\alpha \\
i
\end{array}\right) X_{1}^{n-2}\left(X_{2}+\cdots+X_{N}\right)^{i}
$$


oll sejà, $\left(X_{2}+\cdots+X_{N}\right)^{n}=\left(X_{1}+X_{2}+\cdots+X_{N}\right)^{a}-X_{1}^{a}-\sum_{i=1}^{a-1}\left(\begin{array}{c}a \\ i\end{array}\right) X_{1}^{a-i}\left(X_{2}+\right.$ $\cdots+\left(V_{N}\right)^{2},($ innos

$$
\begin{aligned}
& \left(\begin{array}{c}
\left(X_{2}+X_{3}+\cdots+X_{N}\right)^{a} \\
\left(X_{1}+X_{3}+\cdots+X_{N}\right)^{n} \\
\vdots \\
\left(X_{1}+X_{2}+\cdots+X_{N-1}\right)^{n}
\end{array}\right)=\left(X_{2}+X_{3}+\cdots I_{N}\right)^{a}\left(\begin{array}{c}
1 \\
1 \\
\vdots \\
1
\end{array}\right)-\left(\begin{array}{c}
X_{1}^{\prime} \\
X_{2} \\
\vdots \\
X_{N}^{u}
\end{array}\right) \\
& \sum_{i=1}^{N-1}\left(\begin{array}{c}
\alpha \\
i
\end{array}\right)\left(\begin{array}{c}
X_{1}^{(\alpha-i)}\left(X_{2}+X_{3}+\cdots+X_{N}\right)^{i} \\
X_{2}^{r(\alpha-i)}\left(X_{1}+X_{3}+\cdots+X_{N}\right)^{i} \\
\vdots \\
X_{N}^{(\alpha-i)}\left(X_{1}+X_{2}+\cdots+X_{N-1}\right)^{i}
\end{array}\right)
\end{aligned}
$$

Logo, para cada $r_{1}, \ldots, r_{N}$ podemos escrever

$$
\left(\begin{array}{c}
x_{1}^{\left(n_{1}\right.}\left(x_{2}+x_{3}+\cdots+x_{N}\right)^{x_{2}} \cdots\left(x_{2}^{N-1}+x_{3}^{N-1}+\cdots+x_{N-1}^{N-1}\right)^{n_{N}} \\
x_{2}^{n}\left(x_{1}+x_{3}+\cdots+x_{N}\right)^{x_{2}} \cdots\left(x_{1}^{N 1}+x_{3}^{N-1}+\cdots+x_{N}^{N-1}\right)^{n_{N}} \\
\vdots \\
x_{N}^{\prime 1}\left(x_{1}+x_{2}+\cdots+x_{N-1}\right)^{x_{2}} \cdots\left(x_{1}^{N-1}+x_{2}^{N-1}+\cdots+x_{N-1}^{N-1}\right)^{x_{N}}
\end{array}\right)
$$

como soma de termos da forma

$$
\prod_{k=1}^{N}\left(x_{1}^{k}+\cdots-x_{N}^{k}\right)^{k}\left(\begin{array}{c}
x_{1}^{i} \\
x_{2}^{i} \\
\vdots \\
x_{N}^{i}
\end{array}\right)
$$

para intriros $a, i \geq 0$.

Como sabemess cue

$$
f(x)-\left(x-x_{1}\right)\left(x-x_{2}\right) \ldots\left(x-x_{N}\right)=x^{N}-\sigma_{1} x^{N-1}+o_{2} x^{N-2}+\ldots(-1)^{N} \sigma_{N},
$$

onde os $o_{i}$ 's săo polinomios simétricos clementares e que $f\left(x_{i}\right)=0$ para $t=1, \ldots, N$,

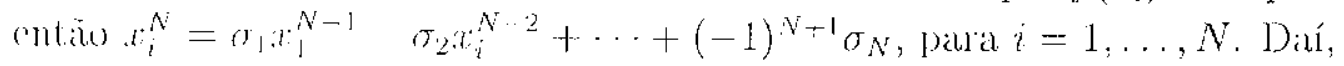

$$
\left(\begin{array}{c}
x_{1}^{N} \\
x_{2}^{N} \\
\vdots \\
x_{N}^{N}
\end{array}\right)-\sigma_{1}\left(\begin{array}{cc}
x_{1}^{N} & 1 \\
x_{2}^{N-1} \\
\vdots \\
x_{N}^{N} & 1
\end{array}\right)-\cdots+(-1)^{N}{ }^{n} \sigma_{N}\left(\begin{array}{c}
1 \\
1 \\
\vdots \\
1
\end{array}\right)
$$

Na igualdate acina, fikendo a multiplicango coordenada a courdenada pelo vetor $\left(\begin{array}{cc}x^{k} & N \\ x_{2}^{k} & N \\ \vdots \\ x_{N}^{k}-N\end{array}\right)$ para $k \geq N$ temos que 


$$
\left(\begin{array}{c}
x_{1}^{k} \\
x_{2}^{k} \\
\vdots \\
x_{N}^{k}
\end{array}\right)=\sigma_{1}\left(\begin{array}{c}
x_{1}^{k-1} \\
x_{2}^{k-1} \\
\vdots \\
x_{N}^{k-1}
\end{array}\right)-\cdots+(-1)^{N+1} \sigma_{N}\left(\begin{array}{c}
x_{1}^{k-N} \\
x_{2}^{k-N} \\
\vdots \\
x_{N}^{k-N}
\end{array}\right)
$$

Assim, os temos da format $\left(\begin{array}{c}x_{1}^{k} \\ x_{2}^{h} \\ \vdots \\ x_{N}^{k}\end{array}\right)$ porkem ser escritus como combinaçäo des elementos clat formal $\left(\begin{array}{c}x_{1}^{h-3} \\ x_{2}^{k}, j \\ \vdots \\ x_{N}^{k \cdots j}\end{array}\right)$, jara $j=1, \ldots, N$, sobre os polinômios simétricos. Se existir $j$ tal cue $h: j \geq N$ repetimus o processo.

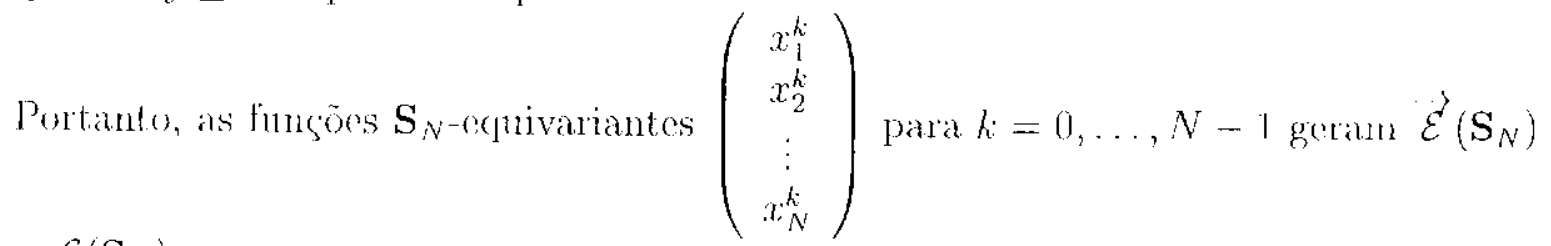
sobre $\mathcal{E}\left(\mathrm{S}_{N}\right)$.

Corolário 3.2.7 O módulo $\overrightarrow{\mathcal{E}}\left(\mathrm{O}(2) \geq \mathrm{S}_{N}\right)$ é gerado sobre o anel $\mathcal{E}\left(\mathrm{O}(2)\right.$ ? $\left.\mathrm{S}_{N}\right)$ por

$$
\left(\begin{array}{c}
\left(z_{1} \bar{z}_{1}\right)^{k} z_{1} \\
\left(z_{2} \bar{z}_{2}\right)^{k} z_{2} \\
\vdots \\
\left(z_{N} \bar{z}_{N}\right)^{k} z_{N}
\end{array}\right),
$$

$h-0,1, \ldots, N \cdot 1$.

Demonstração Polo Le'ma 3.2 .6 e polo Teorema 3.1 .3 segue o resultidu.

\subsection{Diagramas de bifurcação e estabilidade de soluções}

Consideramos o sistema de equaçóes diferenciais ordinárias

$$
\dot{z}=g(z, \lambda)
$$

omle $: \in \mathbb{C}^{N}, \lambda \in \mathbb{R} e g: \mathbb{C}^{N} \times \mathbb{R}, 0 \rightarrow \mathbb{C}^{N}$ ć um germe na origem $\mathrm{O}(2)<\mathrm{S}_{N}$-cutivariante. Supomos $N \geq 3$.

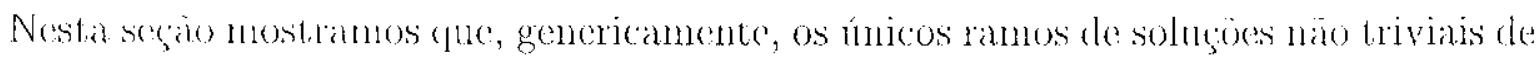
(3.3.8) yue bifuran em $\lambda=0$ são apueles obtidos pelo Lema dos Ramos Equivariantes 
('Jeorema 1.1.1-1). Além disso, estuclamos a estabilidade destes ramos e plotamos os diagramas de bifureacion.

Pelos resultados obtidos na Segĩo 3.2 sabemos que existem polinónios $\mu_{0}, \ldots, p_{N \ldots 1}$ de: $\mathbb{R}^{N}$ ('n $\mathbb{R} \mathbf{O}(2)$; $\mathrm{S}_{N}$-invaliantes tal (jue

$$
g(z, \lambda)=\sum_{k=0}^{N-1} p_{k:}\left(\mu_{1}, \ldots, \mu_{N}, \lambda\right)\left(\begin{array}{c}
\left(z_{1} \bar{z}_{1}\right)^{k} z_{1} \\
\left(z_{2} \bar{z}_{2}\right)^{k} z_{2} \\
\vdots \\
\left(\tilde{z}_{N} \bar{z}_{N}\right)^{k} z_{N}
\end{array}\right),
$$

ande

$$
H_{j}=\left(z_{1} \bar{z}_{1}\right)^{j} ! \cdots+\left(z_{N} \bar{z}_{N}\right)^{\prime}
$$

paral $j=1, \ldots, N$ o $z=\left(z_{1}, \ldots, z_{N}\right) \in \mathbb{C}^{N}$.

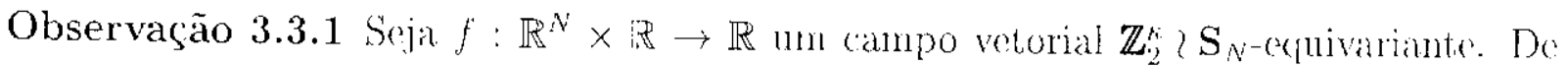
maneira análoga ao que foi feito para obter a lorma (3.3.9) de $g$, obtemos que $f$ ó da forma:

$$
f(x, \lambda)=\sum_{k=0}^{N-1} y_{k}\left(\nu_{i}, \ldots, \nu_{N}, \lambda\right)\left(\begin{array}{c}
\left(x_{1}^{2}\right)^{k} x_{1} \\
\vdots \\
\left(x_{N}^{2}\right)^{k} x_{N}
\end{array}\right),
$$

onde $x=\left(x_{1}, \ldots, x_{N}\right) \in \mathbb{R}^{N}, y_{j}=\left(x_{1}^{2}\right)^{j}+\cdots+\left(x_{N}^{2}\right)^{j}$ para $j=1, \ldots, N$ e $q_{0}, \ldots, q_{N-1}$ são polinomios de $\mathbb{R}^{N+1}$ em $\mathbb{R}^{*} \mathbb{Z}_{2}^{*}\left\langle S_{N^{-}}\right.$-invariantes. Comparando (3.3.9) e (3.3.10) roncluímos

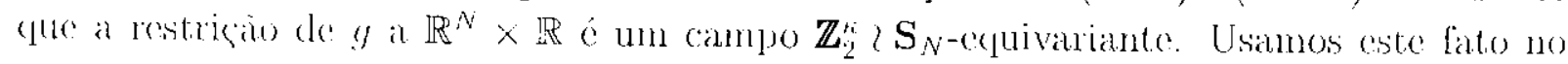
final deste capítulo.

Para $g(a, \lambda)=0$ ser un problema de bifurcasão com simetria $\mathrm{O}(2)$ < $\mathrm{S}_{N}$, devemos axigir $g(0,0)=00(d y)_{0,0}=0$. Para isto, devemos ter

$$
p_{0}(0,0)=0 \text {. }
$$

Trmos une

$$
\Sigma_{\{0, \ldots, b)}=\mathrm{O}(2)<\mathrm{S}_{N}
$$

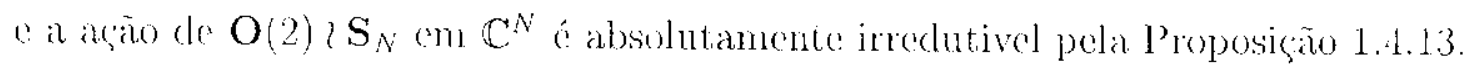

Por (3.3.12), Fix $\left(\mathbf{O}(2)<\mathbf{S}_{N}\right)=\{0\}$. Logo, pela Proposiça 1.4.7 1)), segne (fuc z =0

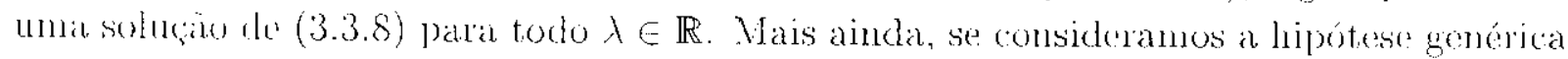

$$
r^{\prime}(0)=\partial \eta_{0} / \partial \lambda(0,0)>0
$$

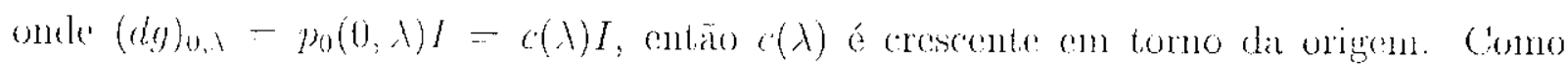
$c(0)=0$, entäe para $\lambda<0, c(\lambda)<0$ e para $\lambda>0, c(\lambda)>0$. Desdo $\left(\right.$ gue $(d g)_{0, \lambda}=c(\lambda) I$, (ntäo $c(\lambda)$ siaro os autovalores de $(d g)_{0, \lambda}$. Logo, $z=0$ é assintoticamente estável para $\lambda<0$

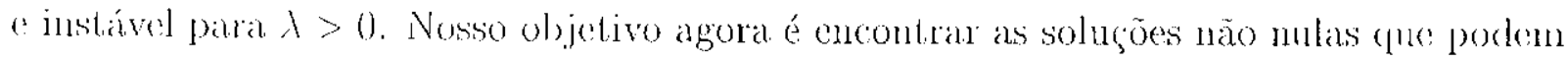
"uarecer desta perda de estabilidade de $z=0$ em $\lambda-0$. Tais solucoes frecuentemente posisucm subgrupos de isotropia 2 menor do que $\mathbf{O}(2)<\mathrm{S}_{N}$.

Iniciancs cucontrando os subgrupos axiais de $\mathbf{O ( 2 )}<\mathbf{S}_{N^{\prime}}$ usando os resultados apresentados 10 Capítulo 2. 
Proposição 3.3.2 Enistem $N$ clusses de conjugaçäo de subırupos axiais para o grupo $\mathrm{O}(2) \geq \mathrm{S}_{N}$ com a acio (1.1.11). Este's subluruos sino:

$$
\Sigma\left(\mathbb{Z}_{2}^{i},\{1,2, \ldots, j\}\right)=\left(\mathbb{Z}_{2}^{i}\right)^{j} \times \mathrm{O}(2)^{N-j}+\mathrm{S}_{3} \times \mathrm{S}_{N-j}
$$

para $j=1, \ldots, N$.

Observação 3.3.3 É imediato que um resultade análogo para o grupo $\mathbb{Z}_{2}^{\kappa}$ ¿ $\mathrm{S}_{N}$ é válido arocando $\mathbb{Z}_{2}^{r}$ jor 1. Este resultado será usado no final deste capítulo (ver da Proposição) $3.3 .8)$.

Demonstração (Proposigño 3.3.2) Cono o nunico sulugrupo axial de $\mathrm{O}(2)$, a menos de conjugagào, é $\mathbb{Z}_{2}^{n}=\{1, \kappa\}$, segue da Proposição 2.3 .1 (ue os subgrupos axiais de

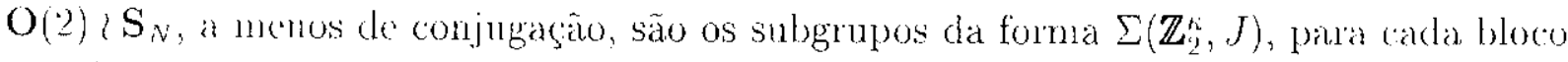
$J \subset\{1,2, \ldots, N\}$. Liscremos

$$
\Sigma_{1}\left(\mathbb{Z}_{2}^{h_{2}}, J\right)-B_{1} \times B_{2} \times \cdots \times B_{N}+Q_{2},
$$

coll

$$
B_{j}=\left\{\begin{array}{l}
\mathbb{Z}_{2}^{\kappa} \text { se } j \in J \\
\mathrm{O}(2) \text { se } j \notin J
\end{array}\right.
$$

$(1$

$$
Q_{J}=\left\{\sigma \in \mathrm{S}_{N}: \sigma(J)=J\right\} .
$$

Isto é, $\Sigma\left(\mathbb{Z}_{2}^{\kappa}, J\right)$ ŕ o produto direto de mm número de cóplas de $\mathbb{Z}_{2}^{r}$, para calda cólula $j \in J$ e cópias de $O(2)$ para as cólulas restantes, todas estendidas por $Q_{3}$. Para qualequer subconjunto de indices de $\{1, \ldots, N\}$, existe um subgrupo $\mathcal{H} \subset \mathrm{S}_{N}$ que age transitivamente nele. Daí, forle subronjunto $J$ de índices de $\{1, \ldots, N\}$ ó um bloco. Tomando $J=\left\{i_{1}, \ldots, i_{p}\right\}$ (om $p \geq 1$, tomos que

$$
Q_{j}=\left(\mathbf{S}_{j} \times \mathbf{S}_{N-p}\right)_{J}
$$

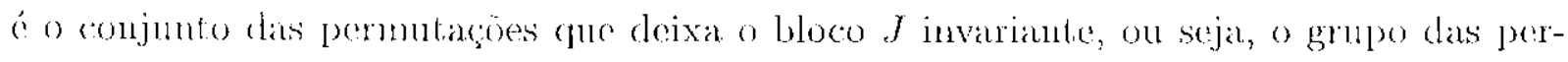
mulagoons de $\mathrm{S}_{N}$ tal que os índices do bloco $J$ são permutados por elementos de $\mathrm{S}_{p}$ e os índices do complementar de $J \mathrm{em}\{1, \ldots, N\}$, digamos $J^{\prime}$, são permutados por elenentos de: $\mathrm{S}_{N-p}$.

$\operatorname{Scjax}=\left(n_{1}, \ldots, n_{N}\right) \in F i x\left(\Sigma\left(\mathbb{Z}_{2}^{i}, J\right)\right)$. Como $x_{j} \in F i x(\mathrm{O}(2))$ se $j \in J^{\prime}$, rutito $x_{j}=0$.

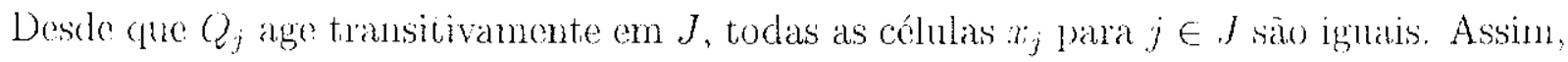
qualeuer posicito con subgrupo de isotropia $\Sigma\left(\mathbb{Z}_{2}^{*}, J\right)$ corresponde a célulats inativas para $j \in J^{\prime}$ e a rélulas ativas e ielenticas para $j \in J$.

Mais aindat se $J_{1}$ o $J_{2}$ säo dois blocos distintos con mesmo número de índices, entào

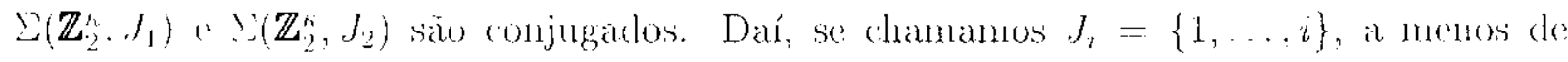

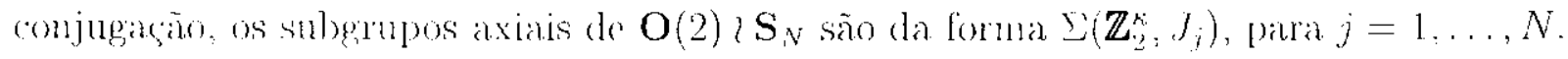




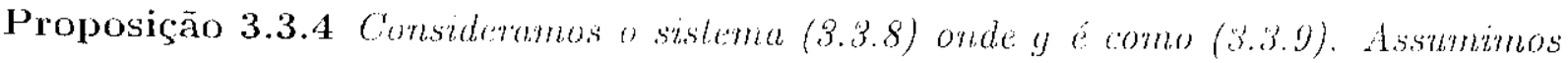

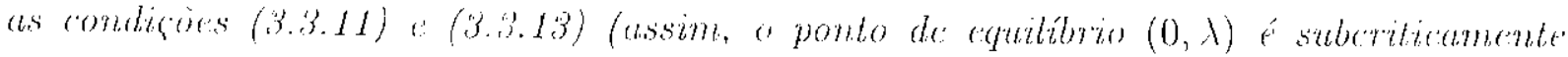

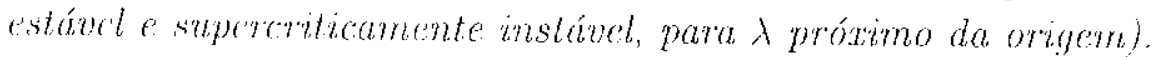

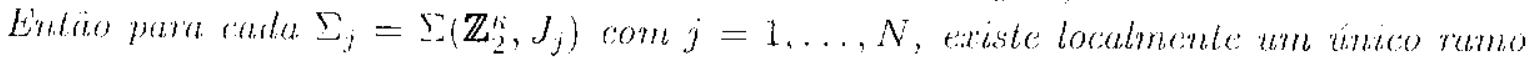
de solucoes para (3.3.8) com tal simetria.

a) () ramo $\Xi_{j}$ é supercritio(suberitico) se $j p_{0, \mu_{1}}(0)+p_{1}(0)<0(>0), p a r a j=1, \ldots, N$.

b) Oramo $\Sigma_{1}$ ir estanel st $j p_{0, t_{1}}(0)+p_{1}(0)<0$ e $\eta_{1}(0)>0$.

c) O ramo $\mathrm{S}_{j}$ para $1<j<N$ a sempre instavel se assumames $p_{1}(0) \neq 0$.

d) Oramo $\beth_{N}$ é estánel so: $N p_{0, \mu_{1}}(0)+p_{1}(0)<0<p_{1}(0)<0$.

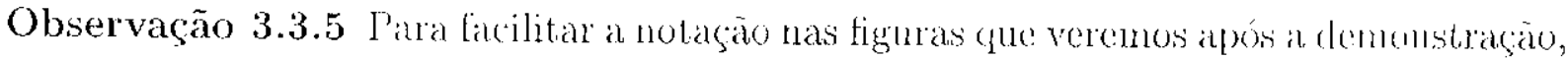

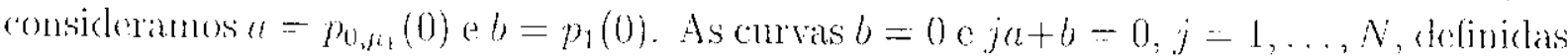
a partir das designaldades dos itens a)-d) acima, definem um conjunto no plano-a,b (que demominanos variedade de transição. Em cada regiăo do plano eletemininalia pela

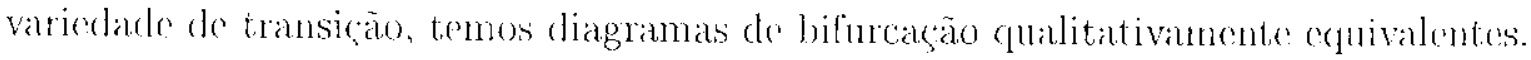

\section{Demonstração (Proposiça 3.3.1)}

a) Com as condiçoses (3.3.11) e (3.3.13) o Lema dos Ramos Equivariantes a firma que para cada $\Sigma_{j}=\Sigma\left(\mathbb{Z}_{2}^{i}, J_{j}\right)$ existe localmente um único ramo de soluçoses com a simetria de tal. subgrupo.

'l'mos (jue

$$
\operatorname{lik}\left(\Sigma_{j}\right)=\{(x, \ldots, \underbrace{x}_{\text {j-ésima posiça }}, 0, \ldots, 0) ; x \in \mathbb{R}\} .
$$

Restringindo g a carbia Fin $\left(\sum_{j}\right)$ e resolvendo a equagano $g-0$, temos:

$$
\begin{gathered}
g((x, \ldots, x, 0, \ldots, 0), \lambda)= \\
p_{0}\left(j x^{2}, j x^{4}, \ldots, j x^{2(N-1)}, \lambda\right)\left(\begin{array}{c}
x \\
\vdots \\
x \\
0 \\
\vdots \\
0
\end{array}\right)+p_{1}\left(j x^{2}, j x^{4}, \ldots, j x^{2(N-1)}, \lambda\right)\left(\begin{array}{c}
x^{3} \\
\vdots \\
x^{3} \\
0 \\
\vdots \\
0
\end{array}\right)+\cdots+ \\
p_{N} 1\left(j x^{2}, j x^{1}, \ldots, j x^{2(N-1)}, \lambda\right)\left(\begin{array}{c}
x^{2(N-1) \cdots 1} \\
\vdots \\
x^{2(N-1)-1-1} \\
0 \\
\vdots \\
0
\end{array}\right)-0 .
\end{gathered}
$$


Colocando $x$ en evidencia en cala linha e considemando $x>0$, tonos:

$$
\begin{gathered}
p_{0}\left(j x^{2}, j x^{1}, \ldots, j x^{2(N-1)}, \lambda\right)+p_{1}\left(j x^{2}, j x^{4}, \ldots, j x^{2(N-1)}, \lambda\right) x^{2}+\cdots+ \\
p_{N-1}\left(j x^{2}, j x^{4}, \ldots, j x^{2(N-1)}, \lambda\right) x^{2(N-1)}=0 .
\end{gathered}
$$

Expandindo $g$ em torno dar origem, tomos

$$
\begin{aligned}
& \left.g(0, \lambda) \equiv p_{0, \mu_{1}}(0) j x^{2}+p_{1, \mu_{1}}(0) j x^{2} x^{2}+\cdots+p_{N-1, \mu_{1}}(0) j x^{2\{N}\right)_{1} x^{2}+p_{0, \lambda}(0) \lambda+ \\
& +p_{1, \lambda}(0) \lambda x^{2}+\cdots+p_{N-1, \lambda}(0) \lambda x^{N-1}+p_{1}(0) x^{2}+\cdots+p_{N-1}(0) x^{2(N \cdots 1)}
\end{aligned}
$$

Numa vizinhança da origem, consideramos os temos de menor ordem:

$$
x^{2}\left(j p_{0, H_{1}}(0)+p_{1}(0)\right)+p_{0, \lambda}(0) \lambda+\ldots .
$$

Assim, a equagào do lano com simetria $\Sigma_{j}$ é dada por

$$
x^{2}\left(j p_{0, k_{1}}(0)+p_{1}(0)\right)+p_{0, \lambda}(0) \lambda-0
$$

011 seja.

$$
\Lambda(x)=-x^{2}\left(j p_{0, \mu, 1}(0)+p_{1}(0)\right) \underbrace{\left(p_{0, \lambda}(0)\right)}_{>0} .
$$

Agmai,

$$
x \Lambda^{\prime}(x)=-2 x^{2}\left(j p_{0, \mu_{1}}(0)+p_{1}(0)\right) \underbrace{\left(p_{0, \lambda}(0)\right)^{-1}}_{>0} .
$$

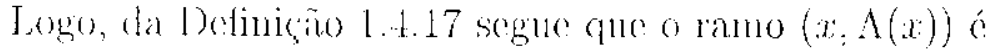

- subcrílicosese jop $p_{0, p_{1}}(0)+p_{1}(0)>0$

- simprecrítico se $j \mu_{0, \mu_{1}}(0)+\mu_{1}(0)<0$.

Se alsismmintos

$$
j p_{0, \mu_{1}}(0)+p_{1}(0) \neq 0,
$$

parra $j=1, \ldots, N$, entäo as diregóes estäo determinadas.

Discutimos agora a estabilidade dos ramos de solugões näo triviais com sinnetria $\Sigma_{y}, j=1, \ldots, N$. Usando as coordenadas $z_{1}, \bar{z}_{1}, \ldots, z_{N}, \bar{z}_{N}$ para $y$ e clemetinuto as componentes de $g$ por $g_{i} \operatorname{com} i=1, \ldots, N$, a derivada da $g$ ó daxla por:

$$
(d g)\left(\begin{array}{c}
u_{1} \\
w_{2} \\
\vdots \\
w_{N}
\end{array}\right)=
$$




$$
\left(\begin{array}{ccccccc}
g_{1 z_{1}} & y_{1 \bar{z}_{1}} & g_{1 z_{2}} & g_{1 \bar{z}_{2}} & \ldots & g_{1 z_{N}} & g_{1 \bar{z}_{N}} \\
g_{2 z_{1}} & y_{2 z_{1}} & g_{2 z_{3}} & g_{2 z_{2}} & \ldots & g_{2 z_{N}} & g_{2 \bar{z}_{N}} \\
\vdots & \vdots & \vdots & \vdots & \ddots & \vdots & \vdots \\
g_{N z_{1}} & g_{N \bar{z}_{1}} & g_{N z_{2}} & g_{N z_{2}} & \ldots & g_{N z_{N}} & g_{N \bar{z}_{N}}
\end{array}\right)\left(\begin{array}{c}
w_{1} \\
w_{1} \\
w_{2} \\
w_{2} \\
\vdots \\
w_{N} \\
\bar{w}_{N}
\end{array}\right) .
$$

Ao longo da solngào trivial $z_{1}=\cdots \cdots z_{N}=0$, tenos

$$
\left(u_{y}\right)_{0, \lambda}\left(\begin{array}{c}
w_{1} \\
w_{2} \\
\vdots \\
w_{N^{\prime}}
\end{array}\right)-w_{0, \lambda}(0, \lambda)\left(\begin{array}{c}
w_{1} \\
w_{2} \\
\vdots \\
w_{N}
\end{array}\right)-\left(\lambda p_{0, \lambda}(0,0)+\ldots\right)\left(\begin{array}{c}
w_{1} \\
w_{2} \\
\vdots \\
w_{N}
\end{array}\right) .
$$

Come já vimos, $(d g)_{0, \lambda}$ é múltiplo da identidade com os autovaloress $\eta_{0}(0, \lambda)$ cujo sinal ó douterminiato por $\lambda p_{0, \lambda}(0)$.

Agora consideramos separadamente os ramos de solucoeses não triviais con simetria $\Sigma_{1}, \Sigma_{j}$ com $1<j<N$ e $\Sigma_{N}$. A razão de separar em casos os subgrmpos $\Sigma_{j}$ e clue para estes tris casos podemos decompor $\mathbb{C}^{N}$ en subespacos invariantes pelas derivadas calculadas em pontos representativos dos subespacos de ponto fixo correspondentes do mancinas ligeinanente diferentes. Para cada subgrupo $\Sigma_{j}$ a a decomposiçá ó

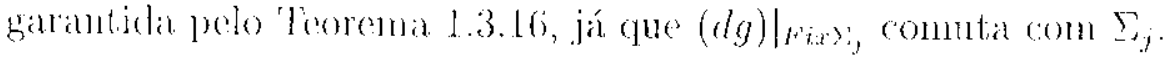

b) Comidermon o ramo de soluçoes $\Sigma_{1}$ e $(x, 0, \ldots, 0) \in F i x \Sigma_{1}$. Temos

$$
\begin{gathered}
(d y) \underbrace{(x, 0, \ldots, 0, \lambda)}_{\left(x_{0}, \cdots\right)}\left(\begin{array}{c}
w_{1} \\
w_{2} \\
\vdots \\
w_{N}
\end{array}\right)= \\
=\left(\begin{array}{c}
g_{1 z_{1}} w_{1}+g_{1 z_{1}} \bar{w}_{1}+g_{z_{2}} w_{2}+g_{1 z_{2}, w_{2}}+\ldots+g_{1 z_{N}} w_{N}+g_{1 \bar{z}_{N}} \bar{w}_{N} \\
g_{21} w_{1}+g_{2 z_{1}} \bar{w}_{1}+g_{2 z_{2}} w_{2}+g_{2 z_{2}} \bar{w}_{2}+\ldots+g_{z_{N}} w_{N}+g_{2 z_{N}} w_{N} \\
\vdots \\
g_{N z_{1}} w_{1}+g_{N \bar{z}_{1}} w_{1}+g_{N z_{2}} w_{2}+g_{N \bar{z}_{2}} \bar{w}_{2}+\ldots+g_{N z_{N}} w_{N}+g_{N \bar{z}_{N}} \bar{w}_{N}
\end{array}\right),
\end{gathered}
$$

com an derivadas parciais das componentes de $y$ avaliadas no ponto $\left(x_{0}, \lambda\right)$.

A expressato acina porte ser simpliticada, resultando om

$$
(d g) \underbrace{(x, 0, \ldots, 0, \lambda)}_{\left(x_{0}, x\right)}\left(\begin{array}{c}
w_{1} \\
w_{2} \\
\vdots \\
w_{N}
\end{array}\right)=\left(\begin{array}{c}
g_{1 n_{1}}\left(x_{0}\right)\left(w_{1}+\bar{w}_{1}\right) \\
p_{0}\left(x^{2}, \ldots, x^{2 N}\right) w_{2} \\
\vdots \\
p_{0}\left(x^{2}, \ldots, x^{2 N}\right) w_{N}
\end{array}\right) .
$$


$\Lambda$ ignaldade $g_{1 z_{1}}\left(x_{0}, \lambda\right)=g_{1 \bar{z}_{1}}\left(x_{0}, \lambda\right)$ segue de $(3.3 .15)$.

A comutatividade de $(d g)_{(r, 0, \ldots, 0, \lambda)} \operatorname{com} \Sigma_{1}$ nos dá uma deromposiçón de $\mathbb{C}^{N}$ em trôs suberpaços, aligamos $I_{0}, V_{1}$, $e V_{2}$, tais que $(d g)_{(x, 0, \ldots, 0, \lambda)}\left(V_{j}\right) \subseteq V_{j}$ para $j=0,1,2$. comsideleramos

$$
r_{0}-\operatorname{ker}(d g)_{(x, 0, \ldots, 0, n)}=\{(i y, 0, \ldots, 0): y \in \mathbb{R}\}
$$

(e tommanos

$$
V_{1}=F i x \Sigma_{1}=\{(y, 0, \ldots, 0) ; y \in \mathbb{R}\}
$$

() subespaco $V_{2}$ c complementan da soma de $f_{0}$ com $V_{b}$, on sejal,

$$
\mathrm{I}_{2}=\left\{\left(0, z_{2}, \ldots, z_{N}\right) ; z_{i} \in \mathbb{C}, i=2, \ldots, N\right\} .
$$

Agora vanos astudar a estabilidade do ramo. Comsideramos $\left.(d g)_{(r, 0, \ldots, 0, \lambda)}\right|_{1 ;}$ :

$$
(d, y)_{(x, 0, \ldots, u, \lambda)}\left(\begin{array}{c}
y \\
0 \\
\vdots \\
0
\end{array}\right)=2 g_{1,21}(x, 0, \ldots, 0, \lambda)\left(\begin{array}{c}
y \\
0 \\
\vdots \\
0
\end{array}\right) .
$$

Logo, $2 g_{1, z}(x, 0, \ldots, 0, \lambda)$ ć um autovalor. Além disso, se consideramos a expansão de $g_{1, i}(x, 0, \ldots, 0, \lambda)$ en tomo da origem e utilizamos a equaça do rano, temos

$$
g_{1, z_{1}}(x, 0, \ldots, 0, \lambda) \cong\left(p_{0, \mu_{1}}(0)+p_{1}(0)\right) x^{2} .
$$

Logo, o sinal de $y_{1, z_{1}}(x, 0, \ldots, 0, \lambda)$ é dado pelo sinal de $p_{0, \mu_{1}}(0)+p_{1}(0)$.

Parar $\left.(t, y)_{(x, u, \ldots, 0, \lambda)}\right|_{1,2}$, temos

$$
(d g)_{(x, 0, \ldots, 0, \lambda)}\left(\begin{array}{c}
0 \\
z_{2} \\
\vdots \\
z_{N}
\end{array}\right)=p_{0}\left(x^{2}, \ldots, x^{2 N}\right)\left(\begin{array}{c}
0 \\
z_{2} \\
\vdots \\
z_{N}
\end{array}\right)
$$

Assine, $p_{0}\left(x^{2}, \ldots, x^{2 N}\right)$ r antovalor de $\left.(d g)_{(x, 0, \ldots, 0, \lambda)}\right\}_{2}$. Se consideramos a expansão de $p_{0}\left(u^{2}, \ldots, x^{2 N}\right)$ em torno da origem e usamos a equagio do ramo, conchumos que

$$
p_{0}\left(x^{2}, \ldots, x^{2 N}\right) \cong-p_{1}(0) x^{2},
$$

on sejia o sinal de $p_{0}\left(x^{2}, \ldots, x^{2 N}\right)$ é determmado $\left.p^{2}\right) \mathrm{r}-p_{1}(0) x^{2}$. Desta forma, conchúmos que o ramo de solugoes com simetria $\Sigma_{1}$ é estável se $p_{0, j}(0)+p_{1}(0)<0$ e $p_{1}(0)>0$. 
c) Para $\Sigma_{j}$ como $1<j<N$, suponhamos que $\left(x_{0_{j}}, \lambda\right)=(x, \ldots, \underbrace{x}_{j-6 \text { sinal }}, 0, \ldots, 0, \lambda) 6$ uma soluçio com $(x, \ldots, x, 0, \ldots, 0) \in F i x \Sigma_{j}$.

Calculando as derivadas parciais das componentes de $g$ no ponto $\left(x_{0_{j}}, \lambda\right)$ obtemos:

$$
(d g)_{\left(x_{0_{j}}, \lambda\right)}\left(\begin{array}{c}
w_{1} \\
w_{2} \\
\vdots \\
w_{N}
\end{array}\right)=\left(\begin{array}{c}
g_{1,21}\left(w_{1}+\bar{w}_{1}\right)+g_{1, z_{2}}\left(w_{2}+\bar{w}_{2}+\cdots+w_{j}+\bar{w}_{j}\right) \\
g_{1, z_{1}}\left(w_{2}+\bar{w}_{2}\right)+g_{1, z_{2}}\left(w_{1}+\bar{w}_{1}+w_{3}+\bar{w}_{3}+\cdots+w_{j} \mid \bar{w}_{j}\right) \\
\vdots \\
g_{1, z_{1}}\left(w_{j}+w_{j}\right)+g_{1, z_{2}}\left(w_{1}+\bar{w}_{1}+\cdots+w_{j, 1}+\bar{w}_{j, 1}\right) \\
p_{0}\left(j x^{2}, \ldots, j x^{2 N}\right) w_{j+1} \\
\vdots \\
p_{0}\left(j x^{2}, \ldots, j x^{2 N}\right) w_{N}
\end{array}\right),
$$

conl $g_{1, \approx 1}$ e $g_{1, z_{2}}$ avaliados em $\left(x_{0_{j}}, \lambda\right)$.

De maneira análoga ao que foi feito para o caso $\Sigma_{1}$, vamos decompor $\mathbb{C}^{N}$ en sulespacos invariantes por $(d g)_{\left(x_{0_{\mathrm{j}}}, \lambda\right)}$ e depois estudar a estabilidade do rano com simetria $\Sigma_{j}, 1<j<N$.

Os subespacos invariantes da clecomposição de $\mathbb{C}^{N}$ são:

$$
\begin{gathered}
V_{u}=\operatorname{lin}(d y)_{\left\langle x_{0_{j}}, \lambda\right)} \ldots\left\{\left(y_{1}, \ldots, i y_{j}, 0, \ldots, 0\right): y_{k} \in \mathbb{R}, k: \cdots 1, \ldots, j\right\}, \\
V_{1}-F u \Sigma_{j}=\{(y, \ldots, y, 0, \ldots, 0): u \in \mathbb{R}\}, \\
V_{2}=\left\{\left(0, \ldots, 0, z_{j+1}, \ldots, z_{N^{\prime}}\right): z_{k} \in \mathbb{C}, k=j_{1}, \ldots, N\right\}
\end{gathered}
$$

('

$$
1_{3}-\mathbb{R}\{(1-j, 1, \ldots, \underbrace{1}_{j \text {-ísima }}, 0, \ldots, 0),(1,1-j, \ldots, 1,0, \ldots, 0), \ldots,(1, \ldots, 1-j, 1,0, \ldots, 0)\} .
$$

Considerando a restriça $\left.(d g)_{\left(x_{0_{\mathrm{j}}}, x\right)}\right|_{v_{1}}$ temos

$$
(d y)_{\left(x_{0_{\mathrm{j}}}, \lambda\right)}\left(\begin{array}{c}
y \\
\vdots \\
y \\
0 \\
\vdots \\
0
\end{array}\right)-\left(2 y_{1, z_{1}}\left(\left(x_{0_{\mathrm{j}}}, \lambda\right)\right)+2(j-1) g_{1, z_{2}}\left(\left(x_{0_{\mathrm{j}}}, \lambda\right)\right)\right)\left(\begin{array}{c}
y \\
\vdots \\
y \\
0 \\
\vdots \\
0
\end{array}\right),
$$

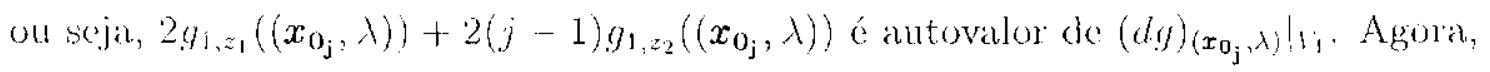

$$
g_{1, z_{1}}\left(\left(x_{0_{j}}, \lambda\right)\right)+(j \cdots 1) g_{1, z_{2}}\left(\left(x_{0_{j}}, \lambda\right)\right) \cong\left(j p_{0, t,}(0)+p_{1}(0)\right) x^{2} .
$$


Já a restriçào $\left.(d g)_{(x, \ldots, x, 0, \ldots, 0, \lambda)}\right|_{r_{2}}$ nos dá $p_{0}\left(j x^{2}, \ldots, x^{2 N}, \lambda\right)$ como autovalor e

$$
p_{0}\left(j x^{2}, \ldots, x^{2 N}, \lambda\right) \cong-p_{1}(0) x^{2}
$$

Da forma rla derivada podemos ter mais informacoes do fue somente decompor $\mathbb{C}^{N}$ cul subesparos invariantes para $(d g)$. De fato, os $j-1$ autovetores escollidos para gerar lis correspondem a un inico antovalor

$$
2\left(y_{1, z_{1}}\left(\left(x_{0_{\mathrm{j}}}, \lambda\right)\right)-y_{1, z_{2}}\left(\left(x_{0_{\mathrm{j}}}, \lambda\right)\right)\right) .
$$

Além disso, usando a equação do ramo temos que o sinel deste antovalor ́́ determinindo por

$$
p_{1}(0)
$$

Conchimes, por (3.3.16) e (3.3.17), que os ramos $\Sigma_{j}, 1<j<N$, säo instáneds para $p_{1}(0) \neq 0$.

d) Para o ramo $\Sigma_{N}$, consideramos $(x, \ldots, x, \lambda)$ uma solucáo con $(x, \ldots, x) \in F a \Sigma_{N}$. Entào

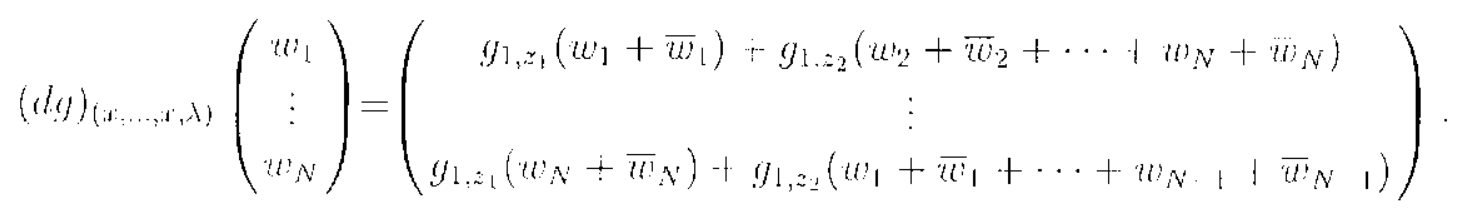

l'odemos decompor $\mathbb{C}^{N}$ em 3 subespaços invariantes por $(d g)_{(x, \ldots, x, \lambda)}$ e a única (ifferenç do item b) ó que náa temos o subespaço comespondente a la.

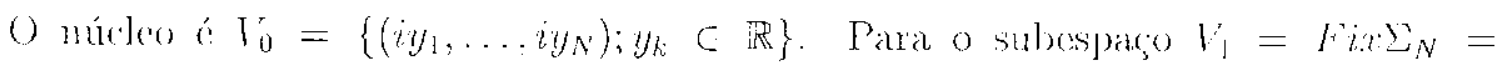
$\{(y, \ldots, y) ; y \in \mathbb{R}\}$ temos o autovalor

$$
2\left(g_{1,21}(x, \ldots, x, \lambda)+(N-1) g_{1, z, 2}(x, \ldots, x, \lambda)\right)
$$

(rijo sinal e determinado por

$$
N p_{0, \mu_{1}}(0)+p_{\ell}(0)
$$

Pana $l_{3}=\mathbb{R}\{(1-N, 1, \ldots, 1), \ldots,(1, \ldots, 1-N, 1)\}$, o único autovalor associado a

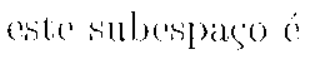

$$
2\left(g_{1,2,}(x, \ldots, x, \lambda)-g_{1,2 ;}(x, \ldots, x, \lambda)\right)
$$

cuju sintal é deteminado por

$$
p_{1}(0)
$$

Conclusio: o ramo $\sum_{N}$ é estável se $N p_{0, \mu_{1}}(0)+p_{1}(0)<0$ : $p_{1}(0)<0$. 


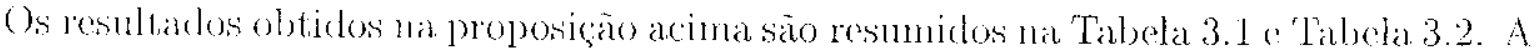
partir destes resultados, obtemos os diagramas de bifurcação correspondentes is soluçoes

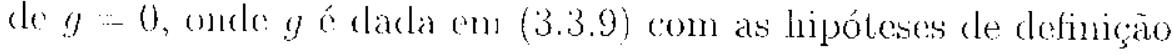

$$
p_{0}(0)=0
$$

o ass hipóteses do nảo degeneraçăo

$$
p_{0, \lambda}(0)>0, p_{1}(0) \neq-j p_{0, \mu}(0), j=1, \ldots, N \circ p_{1}(0)+0
$$

\begin{tabular}{|c|c|c|}
\hline Subgrupos Axiais & Elemonto da orbita & Subespacos invariantes \\
\hline$\sum\left(\mathbb{Z}_{2}^{r}:\{1\}\right)$ & $(x, 0, \ldots,) ; x \in \mathbb{R}$ & $\begin{array}{l}V_{0}=\{(x, 0, \ldots, 0), y \subset \mathbb{R}\} \\
V_{1}=\{(y, 0, \ldots, 0), y \in \mathbb{R}\} \\
V_{2}=\left\{\left(0, z_{2}, \ldots,,_{N}\right), z_{k} \in \mathbb{C}\right\}\end{array}$ \\
\hline $\begin{array}{l}\Sigma\left(\mathbb{Z}_{2},\{1, \ldots, j\}\right) \\
1<j<N\end{array}$ & $(x, \ldots, x, 0, \ldots, 0) ; x \in \mathbb{R}$ & $\begin{aligned} V_{0} & =\left\{\left(i y_{1}, \ldots, i y_{j}, 0, \ldots, 0\right), y_{k} \in \mathbb{R}\right\} \\
V_{1} & -\{(y, \ldots, y, 0, \ldots, 0) ; y \in \mathbb{R}\} \\
V_{2} & -\left\{\left(0, \ldots, 0, z_{j 11}, \ldots, \sigma_{N}\right) ; z_{k} \in \mathbb{C}\right\} \\
V_{3}= & \mathbb{R}\{(1-j, 1, \ldots, 1,0, \ldots, 0), \ldots \\
& (1, \ldots, 1-j, 1,0, \ldots, 0)\}\end{aligned}$ \\
\hline 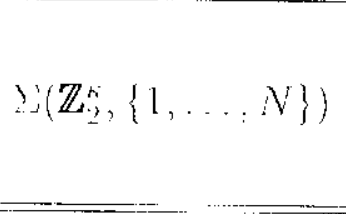 & $(x, \ldots, b) ; x \in \mathbb{C}$ & $\begin{aligned} V_{0} & =\left\{\left(y_{1}, \ldots, i y_{N}\right), y_{k} \in \mathbb{R}\right\} \\
V_{1} & -\{(y, \ldots, y) ; y \in \mathbb{R}\} \\
V_{3} & =(1, \ldots, 1-N, 1)\}\end{aligned}$ \\
\hline
\end{tabular}

Tabrela 3.1: Decomprosigno de $\mathbb{C}^{N}$ em subespacos invariantes por $\Sigma\left(\mathbb{Z}_{2}^{n},\{1, \ldots, j\}\right), 1 \leq$

\begin{tabular}{|c|c|c|}
\hline Subgrupo de isotropia & Equaça do ranno & Autovalores \\
\hline $\mathrm{O}(2)<\mathrm{S}_{N}$ & $z_{1}-\cdots=z_{N}-0$ & $\mu_{0, \lambda} \lambda$ \\
\hline$\Sigma\left(\mathbb{Z}_{2}^{k},\{1\}\right)$ & $\lambda=-\frac{p_{0,4+1}+p_{i}}{p_{0, \lambda}} x^{2}$ & $\begin{array}{l}p_{0 ; p_{1}}+p_{1} \\
p_{1}\end{array}$ \\
\hline $\begin{array}{l}\Sigma\left(\mathbb{Z}_{2}^{n},\{1, \ldots, j\}\right) \\
1<j<N\end{array}$ & $\lambda=-\frac{j p_{0, \mu 1}+\mu_{1}}{p_{0, \lambda}} x^{2}+$ & $\begin{array}{l}m \mu_{0, \mu} \quad 1 p_{1} \\
-p_{1} \\
\mu_{1}\end{array}$ \\
\hline$\therefore\left(\mathbb{Z}_{2}^{t_{2}},\{1, \ldots, N\}\right)$ & $\lambda=-\frac{N p_{0, j 1}+p_{1}}{p_{0, \lambda}} x^{2}+$ & $\begin{array}{l}N p_{0, \mu_{1}}+p_{1}, \\
p_{1}\end{array}$ \\
\hline
\end{tabular}
$j \leq N$

Tabola 3.2: Ramos e estabilidade de soluçōes do problema de bifuncaçäo com simetria $O(2)<S_{N}$ mativamente as sems subgrupos de isotropia

A Figura 3.3, Figuna 3.1, Figma 3.5 e Figura 3.6 representam us diagrantas en cada mua dessas regioes con a estabilidade das solugoes e diregäo dos ranos. Os ramos pon-

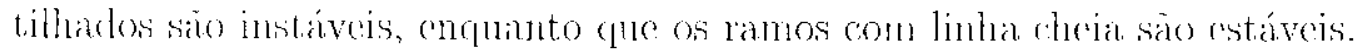




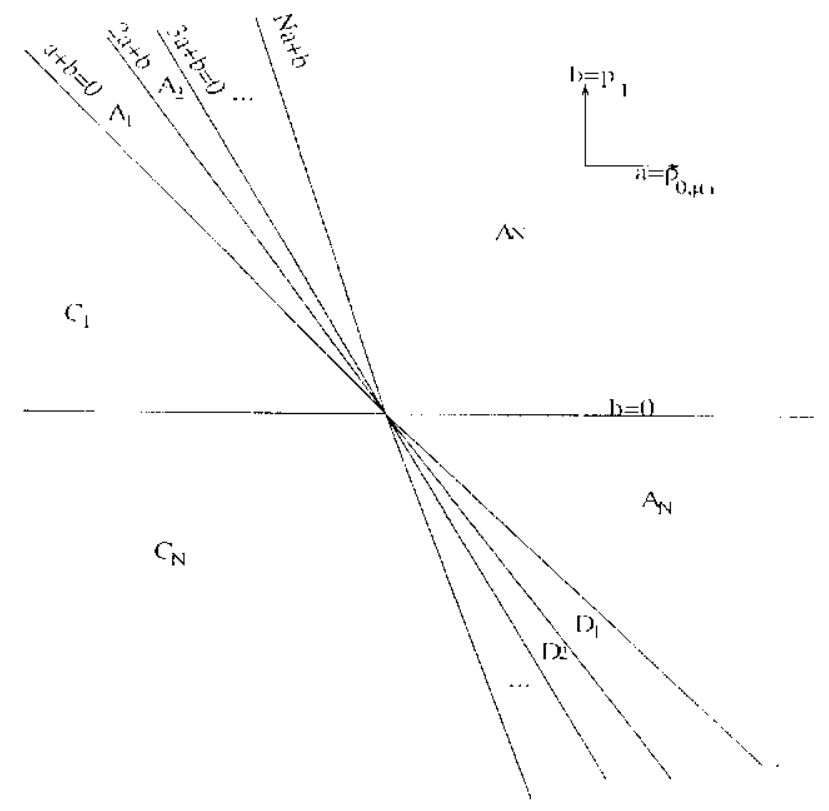

Fignra 3.2: Variedade de transiça

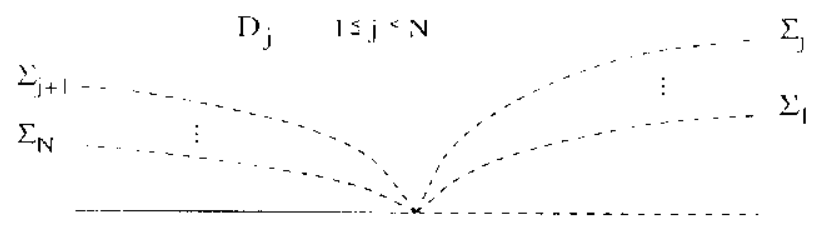

l'igura 3.3: Diagrama para $(a, b) \in D_{j}, 1 \leq j<N$

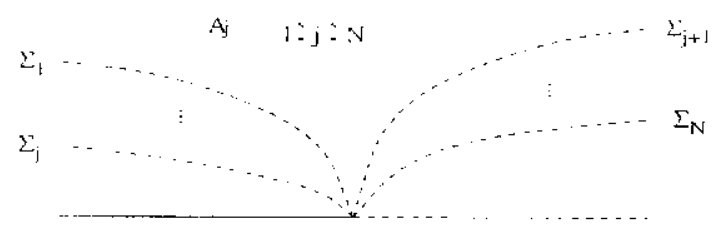

Figrura 3.4: Diagrama para $(a, b) \in A_{j}, 1 \leq j \leq N$ 


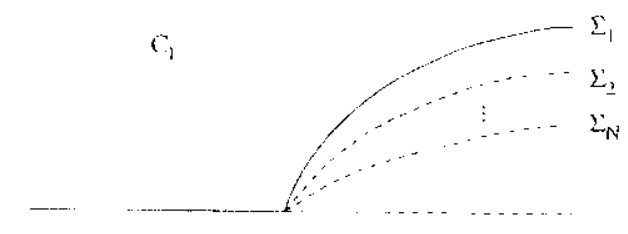

Fignara 3.5: Diagranna para $(a, b) \in C_{1}$

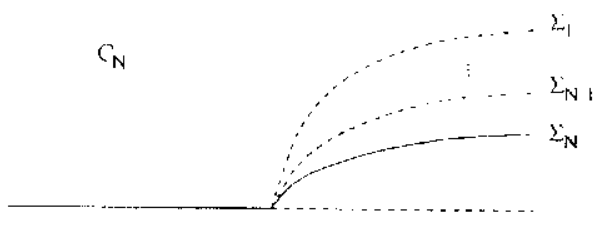

Figura, 3.6: Diagrama para $(a, b) \in C_{N}$

() nusso objedivo agora é verificar que, genericamente, o problena de bifurcagno

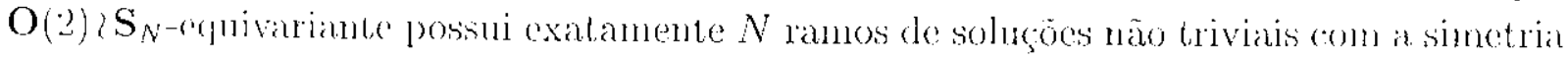
dos subgrupos axiais de $\mathbf{O}(2)$ < $\mathbf{S}_{N}$. Isto é dado na Proposição 3.3.8. Antes, a prosentamos dois lemals a muat conjectural.

Lema 3.3.6 ป̀ é un subgrupo de isolropia maximal de $\mathrm{O}(2)<\mathrm{S}_{N}$ (de $\mathbb{Z}_{2}>\mathrm{S}_{N}$ ) se, e

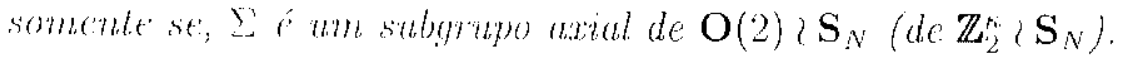

Demonstração Segue direlianonte dà Proposição 2.3.4 e P’oposiçáo 2.3.7.

Lema 3.3.7 Seja $z_{01} \in \mathbb{C}^{N}$ e tomemos $x_{0} \in \mathbb{R}^{N}$ tal que $x_{0} \in \mathbf{O}(2)<\mathbf{S}_{N} \cdot z_{01}$. Se 2 z $z_{0}$ um subyrapo de isolropia submaximal de $z_{0}$ em $\mathrm{O}(2)<\mathrm{S}_{N}$, chtäo $\Sigma_{x_{0}}$ é um subgrupo de isotropia submetamal om $\mathbb{Z}_{2}^{n} 2 \mathbf{S}_{N}$.

Demonstração $\quad S e j a \Sigma_{z_{0}}$ o subgrupo do isotropia de zo submaximal on $O(2)\left\langle S_{N}\right.$.

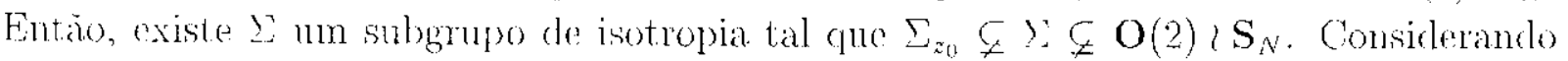
$x_{0}=\gamma \cdot z_{0}$ para algum $\gamma \in \mathrm{O}(2)\left\{\mathrm{S}_{N}\right.$ o $\sum_{x_{0}}$ seu subgrupo do isotropia em $\mathrm{O}(2)<\mathrm{S}_{N}$, temos

$$
\Sigma_{x_{0}}=\gamma \Sigma_{z_{0}} \gamma^{1} \mp \gamma \nu^{-1} \nsubseteq \mathrm{O}(2) i \mathrm{~S}_{N}
$$

oll seja, L' é subgrupo de isotropia submaximal en $\mathrm{O}(2)<\mathrm{S}_{N}$.

Agora vames mostran que $\Sigma_{x_{0}}$ a un sulygrupo de isotropia submaximal em $\mathbb{Z}_{2}^{r_{2}}$ \& $\mathrm{S}_{N}$.

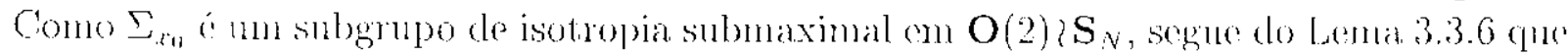

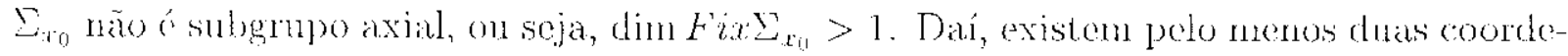

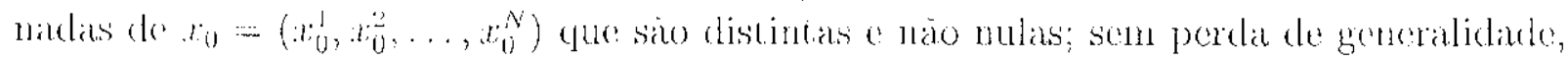
sil]) onsos (jus: $x_{0}^{1} \neq x_{0}^{2}, x_{0}^{1}, x_{t 1}^{2} \neq 0$. 
'Timosictue

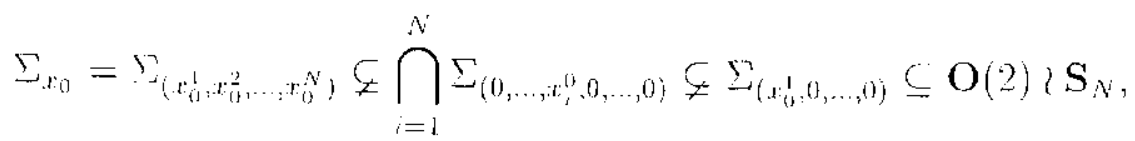

() que intulical

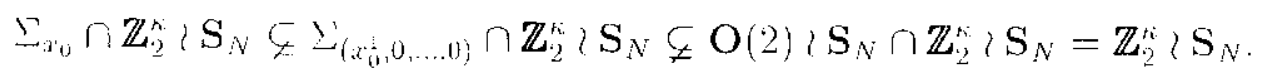

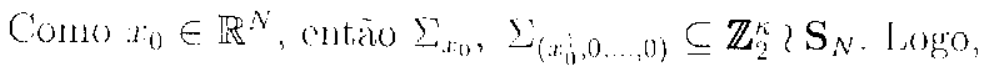

$$
\Sigma_{x, 0} \mp \Sigma_{\left(x_{0}^{1}, 0, \ldots, 0\right)} \nsubseteq \mathbb{Z}_{2}^{i}<\mathrm{S}_{N}
$$

014 seja, $\Sigma_{: 4}$ ó um subgrupo de isotropia submaximal do $\mathbb{Z}_{2}^{\prime}>\mathrm{S}_{N}$.

Conjectura do Subgrupo de Isotropia Maximal (MISC) [0]: Scja $\Gamma$ um gru])o de Lic compacto agindo absolutamente irredutivelnente em $\mathbb{R}^{N}$. Seja $g: \mathbb{R}^{N} \times \mathbb{R} \rightarrow \mathbb{R}^{N}$

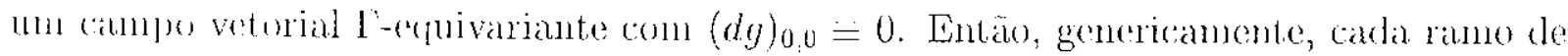
solucoses mio trivial de $g-0$ corresponde a um subgrmpo de isotropia maximal. Nais aincla, cada subgrupo de isotropia maximal corresponde a um ramo de soluçoes do $g=0$ para illgill11 y.

Ein $[6]$ sétí provado que o resultado acima vale para os grupos de Wey] $\mathrm{W}\left(B_{k}\right)$, ou seja, o grupo clas transformagoes do $\mathbb{R}^{k}$ a la forma

$$
\left(x_{1}, x_{2}, \ldots, x_{k}\right)+>\left( \pm x_{\sigma(1)}, \pm x_{\sigma(2)}, \ldots, \pm x_{\sigma(k)}\right)
$$

onde $\sigma$ é mand permutaça con $\mathbf{S}_{k}$. Notamos que $\mathbb{Z}_{2}$ < $\mathbf{S}_{N}$ é um grupo de Weyl $W\left(B_{N}\right)$.

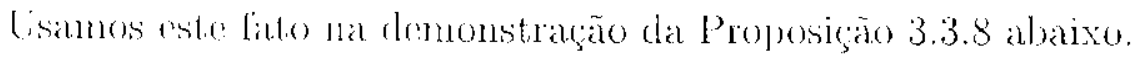

Proposição 3.3 .8 Jm problema de bifuraçăo $\mathrm{O}(2)$ i $\mathrm{S}_{N}$-equmartante possum, genericu-

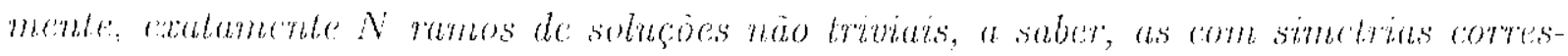
pondentes aos $N$ subgrupos axinis de $\mathrm{O}(2)<\mathrm{S}_{N}$.

Demonstraçāo Considoramos $y: \mathbb{C}^{N} \times \mathbb{R} \rightarrow \mathbb{C}^{N}$ um campo vetorial $\mathrm{O}(2)$ ? $\mathrm{S}_{N^{-}}$ cquivalante, onde $g$ c la forma $(3.3 .9)$, com $z_{0}=\left(z_{0}^{1}, \ldots, z_{0}^{N}\right) \in \mathbb{C}^{N} \circ\left(z_{0}, \lambda\right) \in \mathbb{C}^{N} \times \mathbb{R}$

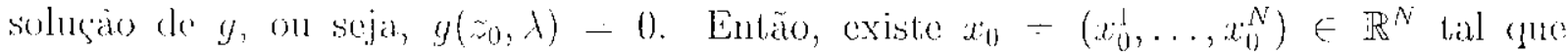

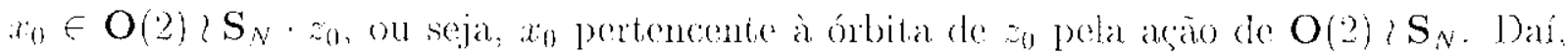
$g\left(x_{0}, \lambda\right)=0$ e portanto, para $f=\left.g\right|_{N^{N} \times k}$, temos $f\left(x_{0}, \lambda\right)-0$. Notanos que $f$ ó $\mathbb{Z}_{?} ; \mathbf{S}_{N^{-}}$

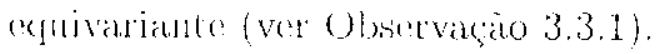

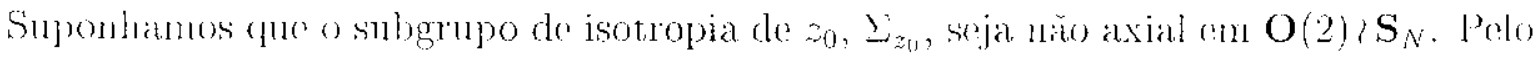

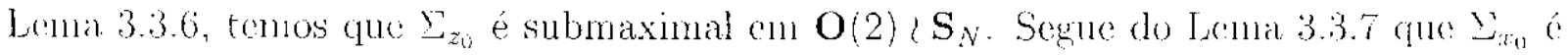

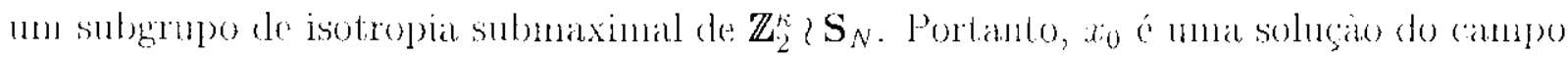

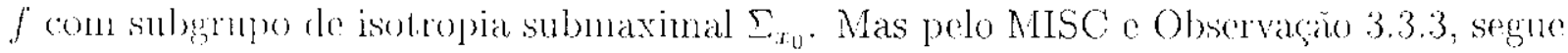


(que, gencricancente, un problema de bifurcação com simetria $\mathbb{Z}_{2}^{\kappa}<S_{N}$ possui, a menos de conjugacio, somente $N$ ramos de soluçoes não triviais com simetrias correspondentes aos $N$ subgrupes de isotropia maximais de $\mathbb{Z}_{2}^{\kappa}<\mathbf{S}_{N}$.

Conclúmos, juntantente com o Lema 3.3 .6 e a Proposiçäo 3.3.2, que as únicas soluçós

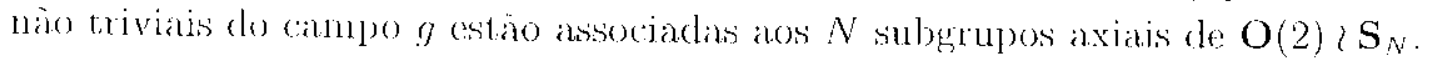




\section{Apêndice A}

\section{Redução de Liapunov-Schmidt}

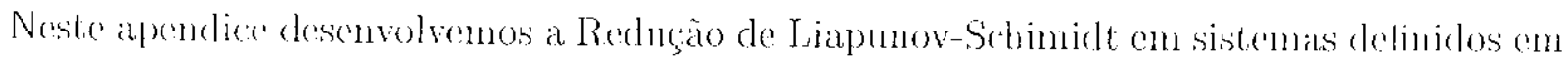
espaços de dimensão infinita quanclo a parte linear do operador tem núcleo do dincmsão tinita. A Proposigio A.0.12 nos garante uma correspondência local entre as soluçós do sistema original com as solugoes do sistema redurido. Além disso, no caso en fue o sistema original possui simetria, a Proposigño $\Lambda .1 .3$ afirma (une o sistema reduziclo pela Redugäo de Liapunov-Schmidt preserva esta simetria. Introduzinos inicialnente alguns

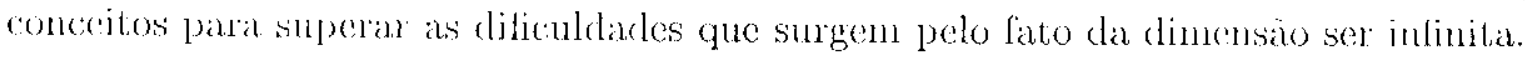

Definiçōes A.0.9 Seja $X$ e $y$ espaços de Banach. Um operudor limilado $L: X \rightarrow y ́$ chamalo Fredholm se a.s seguintes condiçies estâo satisfeitas:

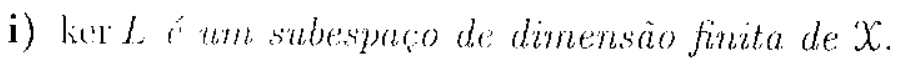

ii) In L é um subespuço fexhado de y de codimensáno finila.

Se L é Frellown, o indice de L é o inteiro

$$
i(L)=\operatorname{dim} \operatorname{ker} L-\operatorname{codin} \ln L
$$

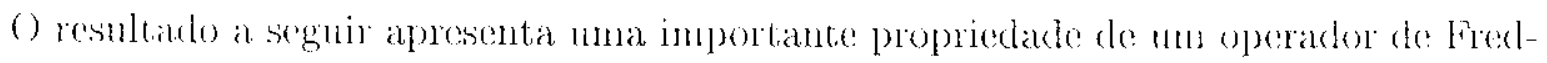
holnt.

Proposição A.0.10 Se $L: X \rightarrow y$ é um operador de Fredholm, cntäo exintum subespaços $M \bullet N$ de $X$ e. respectivamente, tais que

$$
\text { a) } x=k(r) \oplus M, \text { b) } y=N \oplus \operatorname{In} L
$$

Demonstração Berger[1977],, $31.3 \mathrm{~F}$

Observação A.0.11 Se L é um operador do Fredlolm com údice zero, dinker $L=$ $\operatorname{dim} N$. Enn purticular, se ker $L=\{0\}$, entäo $L$ é invertivel. 
Discutimos o nso dos complementos ortogonais em (A.0.1), isto ć,

$$
\begin{aligned}
& \left(\text { a) } M-(\operatorname{ler} L)^{\perp},\right. \\
& \left(\text { b) } N=(\operatorname{Im} L)^{\perp},\right.
\end{aligned}
$$

oncle para 1 mu subespargo $s \subset y$ definimos

$$
S^{\lrcorner}-\left\{u \in y:\langle u, v\rangle=0, \text { para todo } v \in S^{+}\right\} .
$$

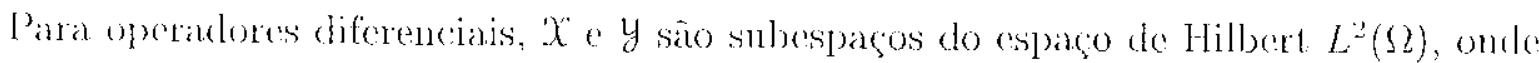
S2 ćm domíno limitado no $\mathbb{R}^{N}$. Este espaço tem o prokluto interno:

$$
\langle u, v\rangle=\int_{\Omega} u(\xi) u(\xi) d \xi
$$

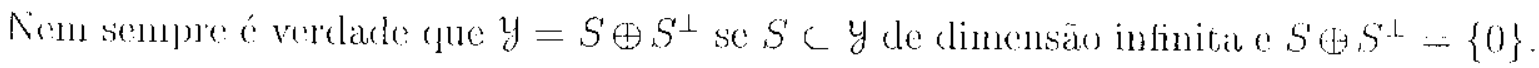
Contude, chía decomposiģa é permitiela quando

a) Strm dinomăo finita

b) Sća imagom de unu operador diferencial elíptico.

No (aso a), a deromposição é obticla pelo processo de ortogonalizaça de Ciram. Schmidt. No (aso b), a discussão cuvolve a alternativa de Fredholm,

$$
(\operatorname{Irn} L)^{-\ldots}=\operatorname{ker} L^{*}
$$

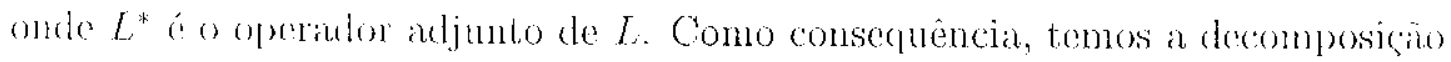

$$
y-(\operatorname{Im} \mathcal{L}) \oplus(\operatorname{Im} L)^{\perp}
$$

(

$$
i(L)=\operatorname{dimker} L+\operatorname{climker} L^{*} .
$$

Apresentamos à seguir o método da rechȩão de Liapunov-Schmiclt e cliscutimus carda

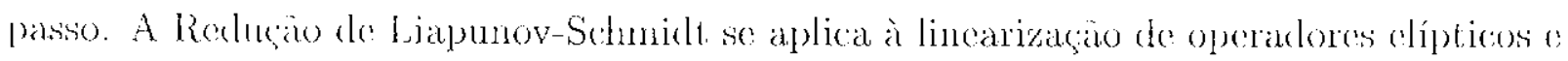
Fredholum dé indice zero.

Sejal

$$
\begin{gathered}
\text { ए: }: x \times \mathbb{R}^{k+1} \rightarrow y \\
\Phi(0,0)=0
\end{gathered}
$$

mma aplicarăo suave entre espagos de Banach. Queremos usar a relução de LiapunovSchmidte para resolver

$$
\Phi(u, \alpha)=0,
$$

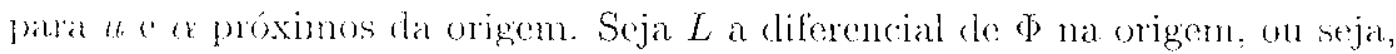

$$
I_{i} u=\lim _{h \rightarrow 0} \frac{\Phi(h u, 0)-\mathrm{D}(0,0)}{h}
$$

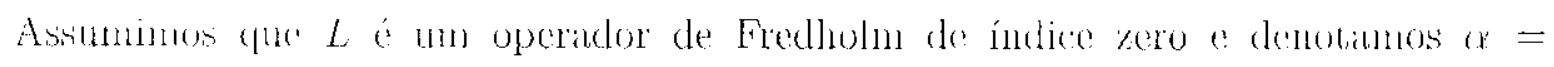
$\left(\alpha_{0}, \alpha_{1}, \ldots, \alpha_{h}\right) \operatorname{com} \alpha_{0}=\lambda \alpha_{1}, \ldots, \alpha_{k}$ parâmetros auxiliares. 
Passo 1 Decompontax $X$ e $y$ como

$$
\begin{aligned}
& \text { a) } x=\operatorname{ker} L \oplus M \\
& \text { b) } y=N \oplus \operatorname{In} L
\end{aligned}
$$

Isto segue da Proposiçio A.0.10, pois L é um opetarlor de Fredholn.

Passo 2 Rerescreva (A.0.6) Conno

$$
\begin{aligned}
& \text { a) } E \Phi(u, \alpha)=0 \\
& \text { b) }(I-E) \Phi\left(u, r^{\prime}\right)=0
\end{aligned}
$$

onde $E: y \rightarrow$ In $L$ é a projeçäo associada à decomposiçín (A.0.7l)).

Passo 3 Use (A.0.7a) para escrever $u=v+w$, onde $v \in$ kn $L$ e $w \in M$. Aplicare Trorema cle Função hmplícita para resolver (A.0.8a) com w como ma funçăo de: " ta. Isto leva a uma funça $W: \operatorname{ker} L \times \mathbb{R}^{k+1} \rightarrow M$ tal que

$$
E \Phi(v+W(v, \alpha), \alpha) \equiv 0
$$

Vamos verilican que de lato podemos aplicar o Teorema da Função Inplícita. Para isto consielerames a aplicagão $F^{\prime}: \operatorname{ker} L \times M \times \mathbb{R}^{k+1} \rightarrow \operatorname{Im} L$ dadia por

$$
F(v, w, \alpha)=L \Phi(v+w, \alpha)
$$

A dilcrencial] de $F$ com respeito a w na origem é:

$$
E \cdot d \Phi_{0,0}=E \cdot L-L
$$

Agora argmentamos que

$$
L: M \rightarrow \operatorname{Im} L
$$

6 invertivel. No caso tinito isto segue pois $L$ restrito a $M$ é bijotola subre a sua inagent. No caso en que os copaços são de Banach. (A.0.11) ́a ainda bijotora, e com a lipótese de que $L$ a Fredholm temos que $L$ é invertívol. Portanto, o Teorema da

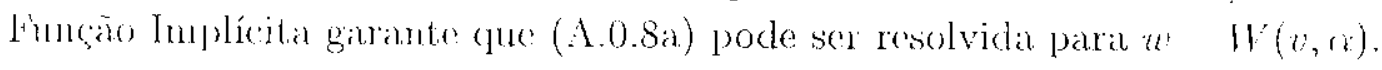

Passo 4 D) (linial $\psi$ : kir $L \times \mathbb{R}^{k+1} \rightarrow N$ por

$$
\phi(v, \alpha)-(I-E) \Phi(v+\Pi(v, \alpha), r) .
$$

Passo 5 Escothat una last: $v_{1}, \ldots, u_{n}$ para $k e r L$ e $v_{1}^{*}, \ldots, v_{n}^{*}$ para (Im $\left.L\right)^{\prime}$. Delina

$$
\begin{aligned}
& y: \mathbb{R}^{n} \times \mathbb{R}^{k+1} \rightarrow \mathbb{R}^{n} \text { por } \\
& \qquad y\left(x,(r)-\left\langle v_{i}^{*}, b\left(x_{1} w_{1}+\cdots+x_{n} v_{w},(r)\right\rangle .\right.\right.
\end{aligned}
$$

Como L G Folholm de índice zero, temos que

$$
\operatorname{dim} \operatorname{ker} L=\operatorname{dim}(\ln L)^{\perp}
$$

(? este mumero ó finito. Assim, as bases para ker $L$ o $(\operatorname{Im} L)^{\perp}$ contem o mesmo mumero do vetores. 
()bormos o seguinte resultarlo:

Proposição A.0.12 Se a lincurizaçăo de (A.0.6) é um operudor de Fredholm de indice

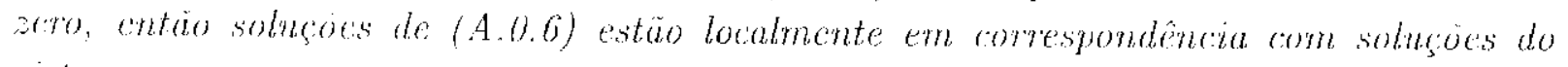
sistrmat

$$
g_{i}(x, \alpha)=0
$$

onde gi, $i-1, \ldots, n$, é drfinidio en (A.0.13).

\section{A.1 A Redução de Liapunov-Schmidt com simetria}

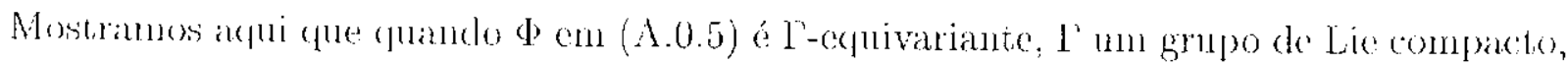

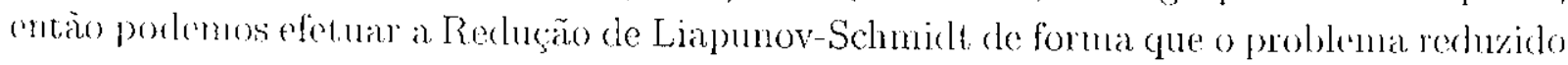
preserve as simetrias de $\Gamma$ do problema original.

Seja $\Gamma$ am grupo de Lie compacto. Dizcnos que $\Gamma$ age no espaço de Banach y be para ada $\gamma \in \Gamma$ existe mun aplicana linear invertivel $R_{\gamma}: y \rightarrow$ y com a propriedade que para toulo $\gamma, \delta \in \Gamma$ '.

$$
R_{\gamma \delta}=R_{\gamma} \circ R_{\delta}
$$

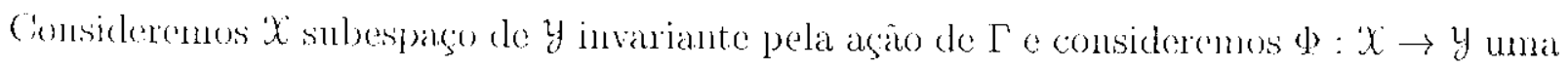
al) licagào que comutia con a agio de $\Gamma$, on seja,

$$
\Phi\left(R_{\gamma} u\right)=R_{\gamma} \Phi(u), \forall u \subset x, \forall \gamma \in \Gamma
$$

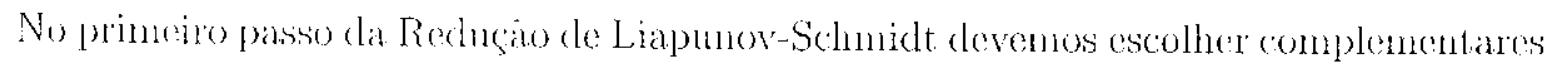
pata certos subespacos de Banach. Quando o sistema possui simetria é importante escother estes subespaços complementares lais que eles sejan $\Gamma$-invariantes. Consideramos os segulintes lates:
a) $\Gamma$ age on $y$
b) $\Phi: x \rightarrow y$ comuta con $\Gamma$
c) $L=(d \Phi)_{0}$ í um opcrador diferencial elíptico.

Mais aindla, precisanos montrar somente os complementos l'-invariantes $M$ e $N$, tais (jul:

$$
\begin{aligned}
& x=\operatorname{ker} L \oplus M, \\
& y=N \oplus \ln I
\end{aligned}
$$

Lema A.1.1 Segum 1, i, $x, y \in L$ como em (A.1.16). Entäo,

a) L comuta com $\mathrm{I}^{\mathrm{T}}$

b) Ker $L$ tum subespugo I'-inuriante de X

c) ln L ó um subespaso I'-invariante de $y$.

\section{Demonstração}


a) Sojan $\gamma \in \Gamma$ e $u \in X$. Lsimos a regra da cadeia para diferenciar a identidade $\Phi(\gamma u)-\gamma \Phi(u)$ e avaliar em $u-0$, obtendo $(d \Phi)_{0} \cdot \gamma=\gamma \cdot(d \Phi)_{0}$, on seja, $L \gamma=\gamma L$.

b) Se $u \in \operatorname{ker} L$, (mation paria todo $\gamma \in \Gamma, L \gamma u=\gamma L u=0$, on soja, $\gamma u \in \operatorname{ker} L$, logo ker $L$ é $\Gamma$-invariante.

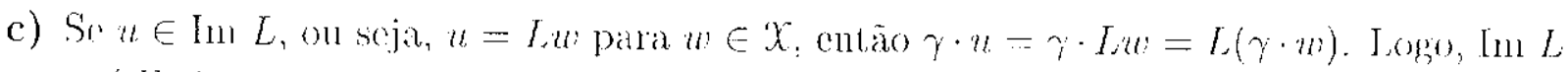
¿ I'-invariante.

Vimos anterionmente que se $L$ é um operador elíptico podemos considerar os complementos (1.1.17)

$$
M=(\operatorname{ker} L)^{-\perp}, N=(\operatorname{Im} L)^{\perp} .
$$

Tumando o produto interno $\langle\cdot, \cdot\rangle$ en $y$ $\Gamma$-invariante. Afrmamos que os complementos

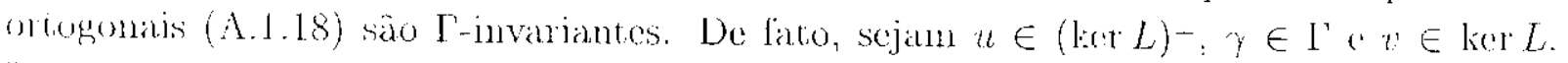
Pelo Lema A.1.1, ker L. Ć T-invariante; logo para qualquer $v \in$ ker $L$, temos $\gamma^{-1}$ we ker $I$. Assin, $0=\left\langle u, \gamma^{-1} v\right\rangle=\langle\gamma u, v\rangle$, ou seja, $(\text { ker } L)^{\perp}$ é $\Gamma$-invariante. Agora, consideramos

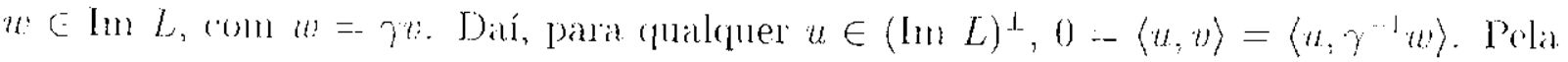
invariancia do produto interno, $\langle\gamma u, w\rangle=0$. I sogo, $(\operatorname{Im} L)^{\perp}$ é $\Gamma$-invariante.

Observação A.1.2 Se o prodıto interno em y nào é preservado por l', porlenus cons-

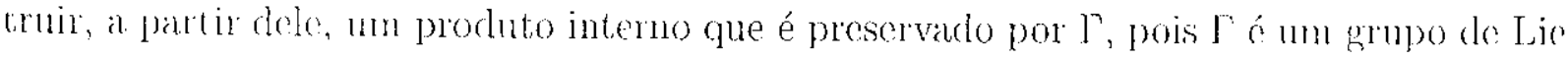
comparto (ver Proposigito 1.1.12).

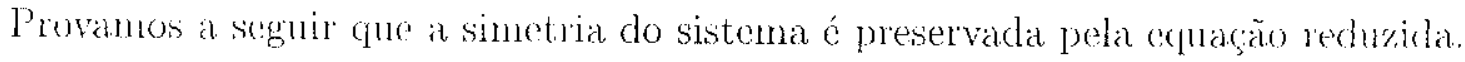
Sijoll

$$
\begin{gathered}
\Phi: x \times \mathbb{R}^{k+1} \rightarrow y \\
\Phi(0,0)=0
\end{gathered}
$$

una aplicacăo suave mtre espaços de Banach. Queremos resolver

$$
\Phi(u, c)=0
$$

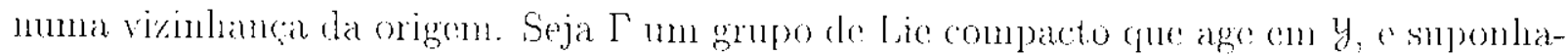
nos $\Phi$ comntando com $\Gamma$. Suponhamos $L=(d \Phi)_{0,0}$ un operador diferencial elíptico de freelholu démelice zero.

A scguninte proposição focaliza a aplicação reduzida $\phi$ obtida no passo 4 . Estar versão de efuacào rechuida é mais conveniente nas análises teóricas, pois ela independe da escolha das coomelenadias do passo 5. Depois de demonstrar a proposigäo discutiremes o passo 5 e as proprediades de simetrial de g.

Proposigăo A.1.3 Na Reductio de Liapunov-Schmidl to (A.1.19), se M t: N an (A.0.7)

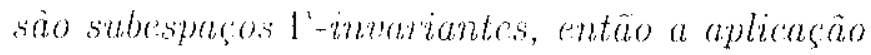

$$
\phi: \operatorname{kn} L \times \mathbb{R}^{k+1} \rightarrow N
$$


definida por (A.0.19) comula com a açio de $\Gamma$, isto

$$
\phi(\gamma u, \alpha)=\gamma \phi(u, \alpha) \forall u \in \operatorname{ker} L, \forall \gamma \in \Gamma
$$

Demonstração Seja $P: y \rightarrow \operatorname{Im} L$ a projeção com lier $E=N$. Queremos (pue $E$ comule com l'. Suponhanos que $u=v+w$ onde $u \in \operatorname{Im} L$ e $w \in N$. Pola lincaridade de Ex ratarion der.

$$
\begin{gathered}
\gamma \cdot E(u)=\gamma E(v+w)=\gamma(E(v)+E(w))=\gamma E(v)=\gamma v=E(\gamma v)=E(\gamma v)+L(\gamma w)= \\
B(\gamma v+\gamma w)=E(\gamma(v+w))=E(\gamma u) .
\end{gathered}
$$

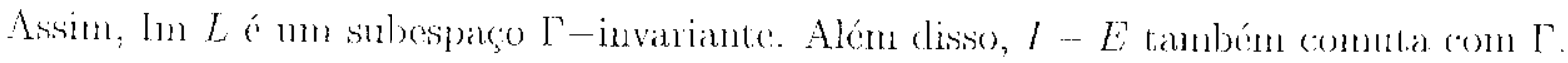
Seja $W: k e r L \times \mathbb{R}^{k+1} \rightarrow M$ a funçào definida em (A.0.9). Afirmamos ane?

$$
W(\gamma \cdot \eta, \alpha)=\gamma H(v, \alpha)
$$

para todo $\gamma \subset \Gamma$. Daí, para $w-v+w$, com $v \in \operatorname{ker} L o w \in M$,

$$
\gamma \cdot \phi(u, \alpha)-\gamma(I-E)\left(u+H^{\prime}(v, \alpha), \alpha\right)=\left(I-E^{\prime}\right)(\gamma v+H(\gamma v, \alpha), \alpha)-\phi(\gamma \cdot v, \alpha) .
$$

Logo, $\phi$ comututal com L'.

Vorifiquemes entaio a valiclade de (A.1.21). Para isto usamos a micidacle elas soluçoes polo Trorema da Funcào Implícita. Fixamos $\gamma \in l^{\prime}$ e definimos:

$$
H,(v, \alpha)-\gamma^{1} W(\gamma \omega, \alpha)
$$

Temos que

$$
E \Phi\left(v+W_{\gamma}(v, \alpha)\right)-E \Phi\left(\gamma^{-1}(\gamma v+W(\gamma v, \alpha))\right)-\gamma^{-1}(E \Phi(\gamma v+W(\gamma v,(v))) .
$$

Como (A.0.9) éváliclo para todo $v \in \operatorname{ker} L$ e ker $L$ é l'-invariante, entiào

$$
E^{\prime} \phi(v+W(v, \alpha))=0 .
$$

Daí, $W_{\gamma}$ também resolve a equaça (A.0.8a) e $W_{\gamma}(0,0)=\gamma^{-1} W(0,0)=0$. Pola unicidake de solugeoses garantida pelo Teorema da Funçào Implícila, concluímos cpue

$$
H^{\prime}(\gamma w, \alpha)-\gamma H^{r}(v, \alpha)
$$

Finalmente, discutimos a escolla das bases para ker $L$ e $(\operatorname{Im} L)^{\perp}$ no passo 5 da Reduça de Liapunov-Sehmidt. Seja $\left\{v_{1}, \ldots, v_{n}\right\}$ und base arbitrária para ker L. Desede que l' age?

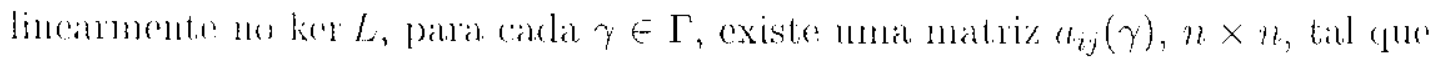

$$
\gamma u_{i}=\sum_{j=1}^{n} a_{i j}(\gamma) u_{i}
$$




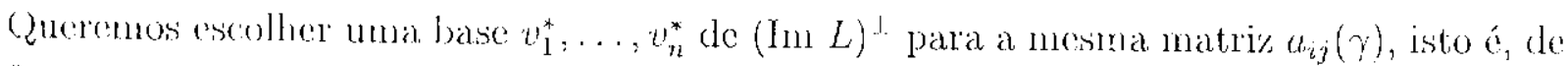
formal anc

$$
\gamma v_{i}^{*}=\sum_{j=1}^{n} a_{i j}(\gamma) v_{j}^{*} .
$$

Falamos encolha consistente das bases se (A.1.22) e (A.1.23) valem simultanca-

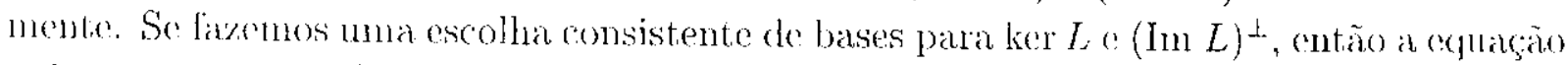
reduzida $g: \mathbb{R}^{n} \times \mathbb{R}^{k} \rightarrow \mathbb{R}^{n}$ vai satisfazcr

$$
g(A(\gamma) x, \alpha)=A(\gamma) g(x, \alpha)
$$

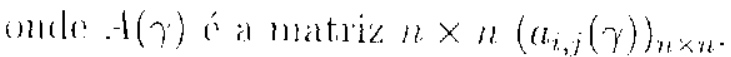

(bualquer representarăo pode ser decomposta na soma direda de representaços irredutíveis (ver Corolário 1.2.3). Agora, a aplicação linear $l$ é un isemorfismo mute $M$ e

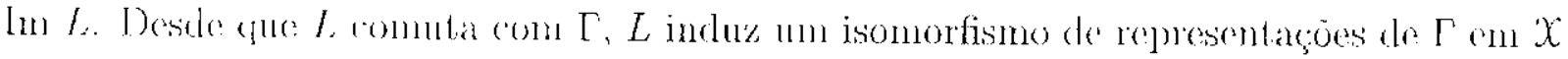
c $y$. Va verdade, as representaçöes de $\Gamma$ cm ker $L$ e $(\operatorname{Im} L)^{\perp}$ devem ser isomorfas. Esta

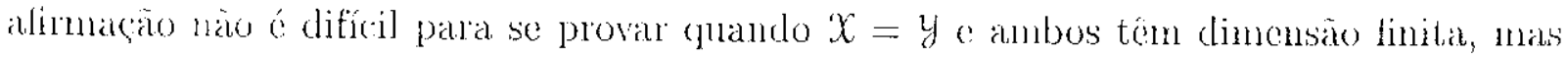
exige mais cuidado quando a dimensão é infinita (ver [8], cap. VII, \$3). Vamos definir "representacoes isomorlas". Scjam $v_{1}, \ldots, v_{n}$ c $v_{1}^{*}, \ldots, v_{n}^{*}$ bases para ker $L$ e $(\operatorname{lm} L)^{\perp}$, respectivamente. Entico definimos as matrizes $A(\gamma)$ cono em $(\Lambda .1 .22)$ e a matriz $B(\gamma)$ a partic de:

$$
\gamma v_{i}^{*} \cdots \sum_{j=1}^{u} b_{i j}(\gamma) v_{j}^{*} .
$$

Dizer cul: as representagoos são isomorfas significa que existe una matriz invertivel $S$ tal (puc, para todo $y \in I^{\prime}$,

$$
B(\gamma)=S^{-1} A(\gamma) S
$$

Portanto, por mona mudanga de base podemos sempre satisfazer (A.1.23).

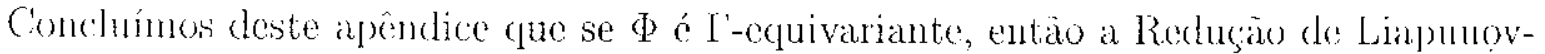

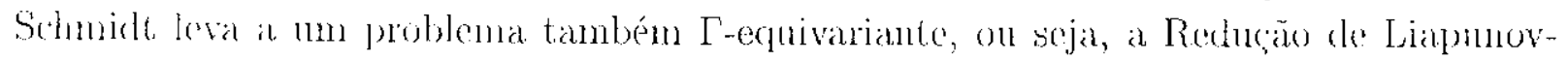
Schmidt prescrvat as simetrias do sistema original. 


\section{Referências Bibliográficas}

[1] Ammitrong, M.A. (1988) Groups and Symmetry (Spring-Vomag, New York)

[2] Colm, P.M. (2000) Clussic Algebra (Wiley, New York).

[3] Jias, A.l'S. (1997) Biluncations with wreath product symmetry. PhD thesis, Universily of Warwick, Inglaterra.

(1) Dionne, 13., Golubitsky, M. and Stewart, I. (1996) Compled cells with internal symmetry. I. Wrath products. Nonlinearity 9 no. 2, 559-574.

[5] Fichl, M. (1996) Lectures on bifurcations, dynamies and symmetry, Research Notes in Mathematics 356 (Lomgman, London).

[6] Field, M and Richardson, R. (1989) Symmetry breaking and maximal isotropy subgroup conjocture lor roflection gromps, Arch. Rational Mech. Anal. 105, 61-94.

[7j Cibson, C.G (1979) Singular Points of Smooth Mapping (Pitman Publishing Limitcol).

[8] Golubitsky, M. and Schaeffer. D. (1984) Singularilies and Groups in Bifurcation Theory Vol, 1, Appl. Math. Sci. 51 (Springer-Verlag, New York).

[9] Golubtsky, M. and Stewart, I. (1986) Hopf bifurcation with dihedral group symmetry: conplexl nonlinear oscillator's. In: Multiparameter Bifuncation Tleory (M. Colubitsky and J. Curkenleinur, Eds.) Contemportry Math 56, 131-173.

[10] Golubitsky, M., Stewart, I. and Schaeffer, D. (1985) Singulantios and Coroups in Bifurcation Theory. Vol. Il, Appl, Math. Sci. 69 (Springer-Verlag, Now York).

11] Hoffunan, K. Énnze, R., (1979) Álgebra linear (Livros Tóenicos e Científicus Editora, Rio de Jilnciros).

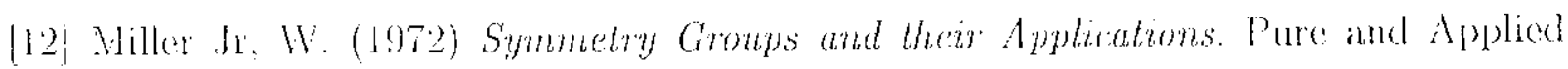
Math. 50 (Acaulemic Press, NY and London).

"13] Strwat. I. (19t5) Gakots Theory (Chapman and Hall. London).

[14] Vau der Warender, B.L. (1948) Modern Algebra Vol. 1 (Frederick Ungar Publishing (C) Now York). 